\title{
EXISTENCE AND STABILITY OF MULTIDIMENSIONAL TRANSONIC FLOWS THROUGH AN INFINITE NOZZLE OF ARBITRARY CROSS-SECTIONS
}

\author{
GUI-QIANG CHEN AND MIKHAIL FELDMAN
}

\begin{abstract}
We establish the existence and stability of multidimensional transonic flows with transonic shocks through an infinite nozzle of arbitrary cross-sections, including a slowly varying de Lavel nozzle. The transonic flow is governed by the inviscid steady potential flow equation with supersonic upstream flow at the entrance, uniform subsonic downstream flow at the infinite exit, and the slip boundary condition on the nozzle boundary. The multidimensional transonic nozzle problem is reformulated into a free boundary problem, for which the free boundary is a transonic shock dividing two phases of $C^{1, \alpha}$ flow in the infinite nozzle, and the equation is hyperbolic in the supersonic upstream phase and elliptic in the subsonic downstream phase. We further develop a nonlinear iteration approach and employ its advantages to deal with such a free boundary problem in the unbounded domain and to solve the multidimensional transonic nozzle problem in a direct fashion. Our results indicate that, for the transonic nozzle problem, there exists a transonic flow such that the flow is divided into a $C^{1, \alpha}$ subsonic flow up to the nozzle boundary in the unbounded downstream region from the supersonic upstream flow by a $C^{1, \alpha}$ multidimensional transonic shock that is orthogonal to the nozzle boundary at every intersection point, and the uniform velocity state at the infinite exit in the downstream direction is uniquely determined by the supersonic upstream flow at the entrance which is sufficiently close to a uniform flow. The uniform velocity state at the exit can not be apriori prescribed from the corresponding pressure for such a flow to exist. We further prove that the transonic flow with a transonic shock is unique and stable with respect to the nozzle boundary and the smooth supersonic upstream flow at the entrance.
\end{abstract}

\section{INTRODUCTION}

We are concerned with the existence and stability of multidimensional steady transonic flows with transonic shocks through general multidimensional infinite nozzles with arbitrary crosssections. Such problems naturally arise in many physical situations, especially in the de Laval nozzles which was first proposed by Swedish engineer Gustav de Lavel in 1887 and has widely been used in the design of steam turbines and modern rocket engines (see Courant-Friedrichs [12], Whitham [48], and the references cited therein). Since the nozzles in applications are usually much longer with respect to their cross-sections, and hence the problem is often formulated mathematically as an infinite nozzle problem. Correspondingly, such a multidimensional infinite nozzle problem has extensively been studied experimentally, computationally, and asymptotically (see $[12,16,19,27,22,23,41,42,48]$ and the references cited therein). Mathematically, the existence and stability of steady transonic flows for such nozzles in a multidimensional setup has been opened since then; see $[5,12,13,44,48]$.

In this paper, we focus on the infinity nozzle to establish the existence and stability of multidimensional transonic flows with supersonic upstream flows at the entrance, uniform subsonic

Date: March 2004.

1991 Mathematics Subject Classification. 35M10,35J65,35R35,76H05,76L05,35B45.

Key words and phrases. Elliptic-hyperbolic, nonlinear equations, second order, mixed type, multidimensional transonic shocks, free-boundary problems, unbounded domains, infinite nozzles, cross-sections, existence, uniqueness, stability, Euler equations, inviscid potential flow. 
downstream flows at the infinite exit, and the slip boundary condition on the nozzle boundary. The potential flow equation for the velocity potential $\varphi: \Omega \subset \mathbf{R}^{n} \rightarrow \mathbf{R}$ is a second-order nonlinear equation of mixed elliptic-hyperbolic type:

$$
\operatorname{div}\left(\rho\left(|D \varphi|^{2}\right) D \varphi\right)=0, \quad x \in \Omega \subset \mathbf{R}^{n},
$$

where the density $\rho\left(q^{2}\right)$ is

$$
\rho\left(q^{2}\right)=\left(1-\theta q^{2}\right)^{\frac{1}{2 \theta}}
$$

and $\theta=\frac{\gamma-1}{2}>0$ with the adiabatic exponent $\gamma>1$.

The nonlinear equation (1.1) is elliptic at $D \varphi$ with $|D \varphi|=q$ if

$$
\rho\left(q^{2}\right)+2 q^{2} \rho^{\prime}\left(q^{2}\right)>0
$$

and hyperbolic if

$$
\rho\left(q^{2}\right)+2 q^{2} \rho^{\prime}\left(q^{2}\right)<0 .
$$

For this infinite nozzle problem, we first seek a multidimensional transonic flow containing a multidimensional transonic shock dividing a subsonic downstream flow from the supersonic upstream flow, motivated by our previous work $[4,5]$. In order to construct such a transonic flow, we first formulate this infinite nozzle problem into a free boundary problem: the multidimensional transonic shock is a free boundary which divides two phases of $C^{1, \alpha}$ flow in the infinite nozzle, and the equation is hyperbolic in the supersonic upstream phase and elliptic in the subsonic downstream phase. Since the existence of the supersonic upstream phase is a direct corollary of the standard local existence theory of the initial-boundary value problem with the slip boundary condition for nonlinear wave equations (cf. [28, 29, 31]), we can further formulate the free boundary problem into a one-phase free boundary problem for a nonlinear elliptic equation by shiffmanization, a cut-off technique (see $[4,46]$ ).

We further develop a nonlinear iteration approach based on [4] and employ its advantages to deal with such a free boundary problem in the unbounded domain and to solve the multidimensional transonic nozzle problem in a direct fashion. Our results indicate that, for the transonic nozzle problem, there exists a transonic flow such that the flow is divided into a $C^{1, \alpha}$ subsonic flow up to the nozzle boundary in the unbounded downstream region from the supersonic upstream flow by a $C^{1, \alpha}$ multidimensional transonic shock that is orthogonal to the nozzle boundary at every intersection point, and the uniform velocity state at the infinite exit in the downstream direction is uniquely determined by the supersonic upstream flow at the entrance which is sufficiently close to a uniform flow. The uniform velocity state at the exit can not be apriori prescribed from the corresponding pressure for such a flow to exist. We further prove that the transonic flow with a transonic shock is unique and stable with respect to the nozzle boundary and the smooth supersonic upstream flow at the entrance.

One of the advantages to employ our nonlinear iteration approach is that, as long as we know how the corresponding fixed conormal problem for (1.1) can be solved and estimated, the solution of the free boundary problem and its estimates directly follow, even for more complicated geometry of the domain under consideration. In this sense, the iteration approach is more efficient than the partial hodograph approach developed in [6], since we do not need to change equation (1.1) with fine features. This indeed plays an important role to achieve the $C^{1, \alpha}$ regularity of both the solution and the free boundary up to the nozzle boundary and to allow the nozzle boundary to have arbitrary cross-sections, by employing the features of equation (1.1). Also, this approach enables us to deal with the multidimensional nozzle problem in a direct fashion, which especially applies to the bounded nozzle problems with various different boundary conditions at the exit by solving the elliptic problems with nonsmooth fixed boundaries, as discussed in Section 3 and in $[10,11,14,24]$.

We point out in passing an important technical difference between the situation considered in this paper and the one in our previous work $[4,5]$. In [5], we considered a transonic flow 
in an infinite cylinder with a cross-section of the form $\Lambda=(0,1)^{n-1}$, i.e., a flow in the domain $(0,1)^{n-1} \times \mathbf{R}$, in which, by reflections, we can reduce the problem to the domain $\mathbf{T}^{n-1} \times \mathbf{R}$, where $\mathbf{T}^{n-1}$ is the $(n-1)$-dimensional torus. Thus, in studying the free boundary problems in $[4,5]$, we were able to avoid studying the intersection of the free boundary with the fixed boundary. In the case of arbitrary smooth cross-section in dimension $n>2$, this can not be done even for the flow in the unperturbed cylinder: indeed, if we locally flatten the boundary, the equation in the new coordinates changes its structure and the reflection leads to an equation with discontinuous coefficients. When we consider a flow in the nozzle (i.e. in a perturbed cylinder), we face a similar problem even in the two-dimensional case. Thus, in the present paper, we have to consider the intersection of a free boundary with a fixed boundary, that is, a nonlinear conormal problem in the domain with nonsmooth boundary.

The uniqueness and stability of the nozzle problem is a question of great importance to know under what circumstances a steady transonic flow involving transonic shocks is uniquely determined by the boundary condition and the conditions at the entrance and when further conditions at the exit are appropriate (see Courant-Friedrich [12]). In this paper, we first identify that the steady transonic flow involving a transonic shock is uniquely determined by the Cauchy data at the entrance for the nonlinear wave equation and the uniform subsonic flow condition at the infinite exit, which are natural physical conditions. Note that the pressure can not be apriori prescribed at the exit; otherwise, it is clear from our results that there is no weak solution for this problem.

Some efforts have been made for solving the nonlinear equation (1.1) of mixed type. In particular, Shiffman [46], Bers [2], and Finn-Gilbarg [18] proved the existence and uniqueness of solutions for the problem of subsonic flows of (1.1) past an obstacle (also see [15]). Morawetz in [43] showed that the flows of (1.1) past the obstacle may contain transonic shocks in general. In $[4,5,6]$, we introduced two approaches to deal with multidimensional transonic shocks. Nontransonic shocks (hyperbolic-hyperbolic shocks) were analyzed in $[7,25,33,40,45,49]$ and the references cited therein.

In Section 2, we set up the infinite nozzle problem involving multidimensional transonic shocks and present the main theorems of this paper. In Section 3, we develop a $C^{1, \alpha}$ estimate framework up to the wedge boundary of the cylinder for the conormal problem for second-order linear elliptic equations of divergence form with $C^{\alpha}$ coefficients for subsequent development. In Section 4 , we reformulate the transonic nozzle problem into a free boundary problem by introducing a multidimensional transonic shock as a free boundary which divides upstream and downstream phases of $C^{1, \alpha}$ flow in the infinite nozzle. We introduce an iteration procedure to construct approximate solutions of the free boundary problem in Section 4 and make uniform estimates of the solutions on the bounded domains with wedge boundary in Section 6. In Section 7, we establish the existence of multidimensional transonic flows with transonic shocks via solving the corresponding free boundary problem in the infinite nozzle. In Section 8, we establish the uniqueness and stability of multidimensional transonic flows with transonic shocks in the infinite nozzle.

The main results in this paper were presented in detail at the International Symposium on Multidimensional Conservation Laws and Related Topics (Shanghai, China), December 19-23, 2003; the Oberwolfach Workshop on Hyperbolic Conservation Laws (Germany), April 4-10, 2004; and the Stanford Workshop on Conservation Laws and Kinetic Theory (USA), July 17-29, 2004.

\section{Transonic Shocks, Nozzle Problem, and Main Theorems}

In this section, we first set up the infinite nozzle problem involving multidimensional transonic shocks and present the main theorems of this paper.

A function $\varphi \in C^{0,1}(\Omega)$ is a weak solution of (1.1) in an unbounded domain $\Omega$ if 
(i) $|D \varphi(x)| \leq 1 / \sqrt{\theta} \quad$ a.e.

(ii) For any $\zeta \in C_{0}^{\infty}(\Omega)$,

$$
\int_{\Omega} \rho\left(|D \varphi|^{2}\right) D \varphi \cdot D \zeta d x=0
$$

We are interested in weak solutions with shocks. Let $\Omega^{+}$and $\Omega^{-}$be open subsets of $\Omega$ such that

$$
\Omega^{+} \cap \Omega^{-}=\emptyset, \quad \overline{\Omega^{+}} \cup \overline{\Omega^{-}}=\bar{\Omega},
$$

and $S=\partial \Omega^{+} \cap \Omega$. Let $\varphi \in C^{0,1}(\Omega)$ be a weak solution of $(1.1)$ and be in $C^{1}\left(\overline{\Omega^{ \pm}}\right)$so that $D \varphi$ experiences a jump across $S$ that is an $(n-1)$-dimensional smooth surface. Then $\varphi$ satisfies the following Rankine-Hugoniot conditions on $S$ :

$$
[\varphi]_{S}=0, \quad\left[\rho\left(|D \varphi|^{2}\right) D \varphi \cdot \nu\right]_{S}=0
$$

where $\nu$ is the unit normal to $S$ from $\Omega^{-}$to $\Omega^{+}$, and the bracket denotes the difference between the values of the function along $S$ on the $\Omega^{ \pm}$sides. Moreover, a function $\varphi \in C^{1}\left(\overline{\Omega^{ \pm}}\right)$, which satisfies $|D \varphi| \leq 1 / \sqrt{\theta},(2.2)$, and equation (1.1) in $\Omega^{ \pm}$respectively, is a weak solution of (1.1), i.e., $\varphi$ satisfies (2.1) in the whole domain $\Omega$.

Set $\varphi^{ \pm}=\left.\varphi\right|_{\Omega^{ \pm}}$. Then we can also write $(2.2)$ as

$$
\varphi^{+}=\varphi^{-} \quad \text { on } S
$$

and

$$
\rho\left(\left|D \varphi^{+}\right|^{2}\right) \varphi_{\nu}^{+}=\rho\left(\left|D \varphi^{-}\right|^{2}\right) D \varphi^{-} \cdot \nu \quad \text { on } S,
$$

where $\varphi_{\nu}^{+}=D \varphi^{+} \cdot \nu$ is the normal derivative on the $\Omega^{+}$side.

Note that the function

$$
\Phi(p):=\left(1-\theta p^{2}\right)^{\frac{1}{2 \theta}} p
$$

is continuous on $[0, \sqrt{1 / \theta}]$ and satisfies

$$
\begin{aligned}
& \Phi(p)>0 \quad \text { for } p \in(0, \sqrt{1 / \theta}), \quad \Phi(0)=\Phi(\sqrt{1 / \theta})=0, \\
& 0<\Phi^{\prime}(p)<1 \text { on }\left(0, c_{*}\right), \quad \Phi^{\prime}(p)<0 \quad \text { on }\left(c_{*}, \sqrt{1 / \theta}\right), \\
& \Phi^{\prime \prime}(p)<0 \text { on }\left(0, c_{*}\right],
\end{aligned}
$$

where

$$
c_{*}=\sqrt{1 /(\theta+1)}=\sqrt{2 /(\gamma+1)}
$$

is the sonic speed, for which a flow is called supersonic if $|D \varphi|>c_{*}$ and subsonic if $|D \varphi|<c_{*}$.

Suppose that $\varphi \in C^{1}\left(\overline{\Omega^{ \pm}}\right)$is a weak solution satisfying

$$
|D \varphi|<c_{*} \quad \text { in } \Omega^{+}, \quad|D \varphi|>c_{*} \quad \text { in } \Omega^{-},\left.\quad D \varphi^{ \pm} \cdot \nu\right|_{S}>0 .
$$

Then $\varphi$ is a transonic shock solution with transonic shock $S$ dividing $\Omega$ into the subsonic region $\Omega^{+}$and the supersonic region $\Omega^{-}$and satisfying the physical entropy condition (see CourantFriedrichs [12]):

$$
\rho\left(\left|D \varphi^{-}\right|^{2}\right)<\rho\left(\left|D \varphi^{+}\right|^{2}\right) \quad \text { along } S .
$$

Note that equation (1.1) is elliptic in the subsonic region and hyperbolic in the supersonic region.

Let $\left(x^{\prime}, x_{n}\right)$ be the coordinates in $\mathbf{R}^{n}$, where $x_{n} \in \mathbf{R}$ and $x^{\prime}=\left(x_{1}, \ldots, x_{n-1}\right) \in \mathbf{R}^{n-1}$. Let $q_{0}^{-} \in\left(c_{*}, 1 / \sqrt{\theta}\right)$ and $\varphi_{0}^{-}(x):=q_{0}^{-} x_{n}$. Then $\varphi_{0}^{-}$is a supersonic solution in $\Omega$. According to $(2.6)-(2.7)$, there exists a unique $q_{0}^{+} \in\left(0, c_{*}\right)$ such that

$$
\rho\left(\left(q_{0}^{+}\right)^{2}\right) q_{0}^{+}=\rho\left(\left(q_{0}^{-}\right)^{2}\right) q_{0}^{-} .
$$


Thus, the function

$$
\varphi_{0}(x)= \begin{cases}q_{0}^{-} x_{n}, & x \in \Omega_{0}^{-}:=\Omega \cap\left\{x: x_{n}<0\right\}, \\ q_{0}^{+} x_{n}, & x \in \Omega_{0}^{+}:=\Omega \cap\left\{x: x_{n}>0\right\}\end{cases}
$$

is a plane transonic shock solution in $\Omega, \Omega_{0}^{+}$and $\Omega_{0}^{-}$are its subsonic and supersonic regions respectively, and $S=\left\{x_{n}=0\right\}$ is a transonic shock.

Defining $\varphi_{0}^{+}(x):=q_{0}^{+} x_{n}$ in $\Omega$, we have

$$
\varphi_{0}(x)=\min \left(\varphi_{0}^{+}(x), \varphi_{0}^{-}(x)\right) \quad \text { for } x \in \Omega .
$$

In this paper, we focus on the following infinite nozzle $\Omega$ with arbitrary smooth cross-sections:

$$
\Omega=\Psi(\Lambda \times(-\infty, \infty)) \cap\left\{x_{n} \geq-1\right\},
$$

where $\Lambda \subset \mathbf{R}^{n-1}$ is an open bounded connected set with a smooth boundary, and $\Psi: \mathbf{R}^{n} \rightarrow \mathbf{R}^{n}$ is a smooth map, which is close to the identity map. For simplicity, we assume that

$$
\partial \Lambda \text { is in } C^{\left[\frac{n}{2}\right]+3, \alpha}
$$

and

$$
\|\Psi-I d\|_{\left[\frac{n}{2}\right]+3, \alpha, \mathbf{R}^{n}} \leq \sigma
$$

for some $\alpha \in(0,1)$ and small $\sigma>0$, where $[s]$ is the integer part of $s, I d: \mathbf{R}^{n} \rightarrow \mathbf{R}^{n}$ is the identity map, $\partial_{l} \Omega:=\Psi(\partial \Lambda \times(-\infty, \infty)) \cap\left\{\left(x^{\prime}, x_{n}\right): x_{n}>-1\right\}$, and $\|u\|_{m, \alpha, \mathcal{D}}$ is the norm in the Hölder space $C^{m, \alpha}(\overline{\mathcal{D}})$ in the domain $\mathcal{D}$. Such nozzles especially include the slowly varying de Laval nozzles $[12,48]$. For concreteness, we also assume that there exists $L>L_{0}$ (say, $L_{0}>10$ without loss of generality) such that

$$
\Psi(x)=x \quad \text { for any } x=\left(x^{\prime}, x_{n}\right) \text { with } x_{n}>L,
$$

that is, the nozzle slowly varies locally in a bounded domain as the de Laval nozzles.

In the two-dimension case, the domain $\Omega$ defined above has the following simple form:

$$
\Omega=\left\{\left(x_{1}, x_{2}\right): x_{1} \geq-1, f_{-}\left(x_{2}\right)<x_{1}<f_{+}\left(x_{2}\right)\right\},
$$

where $\left\|f_{ \pm}-d_{ \pm}\right\|_{4, \alpha, \mathbf{R}} \leq \sigma$ and $f_{ \pm} \equiv d_{ \pm}$on $[L, \infty)$ for some constants $d_{ \pm}$satisfying $d_{+}>d_{-}$. For the multidimensional case, the geometry of the nozzles is much richer.

Note that our setup implies that

$$
\partial \Omega=\overline{\partial_{o} \Omega} \cup \partial_{l} \Omega
$$

with

$$
\begin{aligned}
& \partial_{l} \Omega:=\Psi[\partial \Lambda \times(-\infty, \infty)] \cap\left\{\left(x^{\prime}, x_{n}\right): x_{n}>-1\right\}, \\
& \partial_{o} \Omega:=\Psi(\Lambda \times(-\infty, \infty)) \cap\left\{\left(x^{\prime}, x_{n}\right): x_{n}=-1\right\} .
\end{aligned}
$$

Then our transonic nozzle problem can be formulated into the following form:

Problem (TN): Transonic Nozzle Problem. Given the supersonic upstream flow at the entrance $\partial_{o} \Omega$ :

$$
\varphi=\varphi_{e}^{-}, \varphi_{x_{n}}=\psi_{e}^{-} \quad \text { on } \partial_{o} \Omega
$$

the slip boundary condition on the nozzle boundary $\partial_{l} \Omega$ :

$$
\partial_{\nu} \varphi=0 \quad \text { on } \partial_{l} \Omega
$$

and the uniform subsonic flow condition at the infinite exit $x_{n}=\infty$ :

$$
\left\|\varphi(\cdot)-\omega x_{n}\right\|_{C^{1}\left(\Omega \cap\left\{x_{n}>R\right\}\right)} \rightarrow 0 \quad \text { as } R \rightarrow \infty, \text { for some } \omega \in\left(0, c_{*}\right),
$$

find a multidimensional transonic flow $\varphi$ of the problem (1.1) and (2.18)-(2.20) in $\Omega$. 
As we will see below, the supersonic upstream part $\varphi^{-}$of the solution can be constructed in $\Omega_{1}:=\Omega \cap\left\{x_{n}<1\right\}$ from the data on the nozzle entrance (2.18) by using the standard results on initially-boundary value problems for hyperbolic equations. Thus, assuming that the $C^{1, \alpha}$ supersonic solution $\varphi^{-}$is given in $\Omega_{1}$, we can formulate the transonic nozzle problem (TN) as the following one-phase free boundary problem.

Problem (FB): Free Boundary Problem. Given a supersonic upstream flow $\varphi^{-}$, a weak solution of (1.1) in $\Omega_{1}$, which is a $C^{1, \alpha}$ perturbation of $\varphi_{0}^{-}$:

$$
\left\|\varphi^{-}-\varphi_{0}^{-}\right\|_{1, \alpha, \Omega_{1}} \leq C_{0} \sigma
$$

with $\sigma>0$ small, for some constant $C_{0}$, and satisfies (2.19), find a multidimensional subsonic flow $\varphi^{+}$of (1.1) satisfying (2.19)-(2.20) and identify a free boundary $x_{n}=f\left(x^{\prime}\right)$ dividing the subsonic flow $\varphi^{+}$from the given supersonic flow $\varphi^{-}$so that

$$
\varphi(x)= \begin{cases}\varphi^{+}(x), & x_{n}>f\left(x^{\prime}\right) \\ \varphi^{-}(x), & x_{n}<f\left(x^{\prime}\right)\end{cases}
$$

is a transonic shock solution.

Note that, for a solution $\varphi$ of Problem (FB), the subsonic region $\Omega^{+}$and supersonic region $\Omega^{-}$ in (2.10) are of the form

$$
\Omega^{+}(\varphi)=\left\{x_{n}>f\left(x^{\prime}\right)\right\}, \quad \Omega^{-}(\varphi)=\left\{x_{n}<f\left(x^{\prime}\right)\right\} .
$$

Our main theorem for the free boundary problem, Problem (FB), is the following.

Theorem 2.1 (Existence). There exist $\sigma_{0}>0, C$, and $\hat{C}$, depending only on $n, \alpha, \gamma, q_{0}^{+}$, $C_{0}, \Lambda$, and $L$, such that, for every $\sigma \in\left(0, \sigma_{0}\right)$, any map $\Psi$ satisfying $(2.16)$ and $(2.17)$, and any supersonic upstream flow $\varphi^{-}$of (1.1) satisfying (2.18) and (2.19), there exists a solution $\varphi \in C^{0,1}(\Omega) \cap C^{\infty}\left(\Omega^{+}\right)$of Problem (FB) such that

$$
\left\|D \varphi-q_{0}^{+} e_{n}\right\|_{0,0, \Omega^{+}} \leq \hat{C} \sigma .
$$

Moreover, the solution $\varphi$ satisfies the following properties:

(i) The constant $\omega$ in (2.20) must be $q^{+}$:

$$
\omega=q^{+}
$$

where $q^{+}$is the unique solution in the interval $\left(0, c_{*}\right)$ of the equation

$$
\rho\left(\left(q^{+}\right)^{2}\right) q^{+}=Q^{+}
$$

with

Thus $\varphi$ satisfies

$$
Q^{+}=\frac{1}{|\Lambda|} \int_{\partial_{o} \Omega} \rho\left(\left|D \varphi^{-}\right|^{2}\right) D \varphi^{-} \cdot \nu d \mathcal{H}^{n-1} .
$$

$$
\left\|\varphi-q^{+} x_{n}\right\|_{C^{1}\left(\Omega \cap\left\{x_{n}>R\right\}\right)} \rightarrow 0 \quad \text { as } R \rightarrow \infty,
$$

and $q^{+}$satisfies

$$
\left|q^{+}-q_{0}^{+}\right| \leq C \sigma
$$

(ii) The function $f\left(x^{\prime}\right)$ in (2.22) satisfies

$$
\|f\|_{1, \alpha, \mathbf{R}^{n-1}} \leq C \sigma
$$

and the surface $S=\left\{\left(x^{\prime}, f\left(x^{\prime}\right)\right): x^{\prime} \in \mathbf{R}^{n-1}\right\} \cap \Omega$ is orthogonal to $\partial_{l} \Omega$ at every intersection point;

(iii) Furthermore, $\varphi \in C^{1, \alpha}\left(\overline{\Omega^{+}}\right)$with

$$
\left\|\varphi-q^{+} x_{n}\right\|_{1, \alpha, \Omega_{+}} \leq C \sigma .
$$


If the supersonic upstream flow $\varphi^{-}$has a higher regularity, then we have the following uniqueness theorem.

Theorem 2.2 (Uniqueness). There exists a constant $\sigma_{0}>0$ depending only on $n, \alpha, \gamma, C_{0}, \Lambda$, $L$, and $q_{0}^{+}$such that, if $\sigma<\sigma_{0}$ and the supersonic solution $\varphi^{-}$in Problem (FB) additionally satisfies

$$
\left\|\varphi^{-}-\varphi_{0}^{-}\right\|_{2, \alpha, \Omega_{1}} \leq C_{0} \sigma
$$

then the solution $\varphi$ of Problem (FB) satisfying (2.24) is unique.

The standard local existence theory of smooth solutions for the initial-boundary value problem (2.18)-(2.19) for second-order quasilinear hyperbolic equations implies (see Appendix) that, as $\sigma$ is sufficiently small in (2.16) and (2.21), there exists a supersonic solution $\varphi^{-}$of (1.1) in $\Omega_{1}$, which is a $C^{k+1}$ perturbation of $\varphi_{0}^{-}$: For any $\alpha \in(0,1]$ :

$$
\left\|\varphi^{-}-\varphi_{0}^{-}\right\|_{k, \alpha, \Omega_{1}} \leq C_{0} \sigma, \quad k=1,2,
$$

for some constant $C_{0}>0$, and satisfies

$$
\partial_{\nu} \varphi^{-}=0 \quad \text { on } \quad \partial_{l} \Omega_{1}
$$

provided that $\left(\varphi_{e}^{-}, \psi_{e}^{-}\right)$on $\partial_{o} \Omega$ satisfying

$$
\left\|\varphi_{e}^{-}-q_{0}^{-} x_{n}\right\|_{H^{s+k}}+\left\|\psi_{e}^{-}-q_{0}^{-}\right\|_{H^{s+k-1}} \leq \sigma, \quad k=1,2,
$$

for some integer $s>n / 2+1$ and the compatibility conditions up to the $(s+k)^{t h}$-order, where the norm $\|\cdot\|_{H^{s}}$ is the Sobolev norm with $H^{s}=W^{s, 2}$. In particular, as a direct corollary of Theorem 2.1, Theorem 2.2, and Proposition A.1 in Appendix, we obtain the following existence and uniqueness result for the transonic nozzle problem, Problem (TN):

Theorem 2.3. Let $q_{0}^{+} \in\left(0, c_{*}\right)$ and $q_{0}^{-} \in\left(c_{*}, 1 / \sqrt{\theta}\right)$ satisfy (2.11), and let $\varphi_{0}$ be the transonic shock solution (2.12). Then there exist $\sigma_{0}>0, C$, and $\hat{C}$, depending only on $n, \alpha, \gamma, q_{0}^{+}, \Lambda$, and $L$ such that, for every $\sigma \in\left(0, \sigma_{0}\right)$, any map $\Psi$ satisfying (2.16) and (2.17), and any supersonic upstream flow $\left(\varphi_{e}^{-}, \psi_{e}^{-}\right)$on $\partial_{o} \Omega$ satisfying

$$
\left\|\varphi_{e}^{-}-q_{0}^{-} x_{n}\right\|_{H^{s+2}}+\left\|\psi_{e}^{-}-q_{0}^{-}\right\|_{H^{s+1}} \leq \sigma
$$

for $s>n / 2+1$ and the compatibility conditions up to the $(s+2)^{t h}$-order, there exists a unique solution $\varphi \in C^{0,1}(\Omega)$ of Problem (TN) satisfying (2.23),

$$
\left\|\varphi-\varphi_{0}^{-}\right\|_{2, \alpha, \Omega^{-}} \leq C \sigma,
$$

and (2.24). Moreover, this solution satisfies $\varphi \in C^{0,1}(\Omega) \cap C^{\infty}\left(\Omega^{+}\right)$and properties (i)-(iii) of Theorem 2.1, where (2.27) is replaced by

$$
Q^{+}=\frac{1}{|\Lambda|} \int_{\partial_{o} \Omega} \rho\left(\left|D_{x^{\prime}} \varphi_{e}^{-}\right|^{2}+\left(\psi_{e}^{-}\right)^{2}\right) \psi_{e}^{-} d \mathcal{H}^{n-1} .
$$

Remark 2.1. The smoothness conditions (2.15) and (2.16) are just for simplicity of presentation and can be relaxed: For example, in Theorems 2.1 and 2.2, we require only $C^{2, \alpha}$ in (2.15) and (2.16). The localization condition (2.17) is not essential to achieve Theorem 2.1; In general, it can be replaced by an appropriate decay condition.

Remark 2.2. When the initial data $\left(\varphi_{e}^{-}, \psi_{e}^{-}\right) \equiv\left(-\psi_{e}^{-}, \psi_{e}^{-}\right)$is constant and the nozzle $\Omega \cap\{-1 \leq$ $\left.x_{n} \leq-1+\epsilon\right\}=\Lambda \times[-1,-1+\epsilon]$ for some $\epsilon>0$ as a de Laval nozzle, then the compatibility conditions are automatically satisfied. In fact, in this case, $\varphi^{-}(x)=\psi_{e}^{-} x_{n}$ is a solution near $x_{n}=-1$ in the nozzle. 
Remark 2.3. When $n=2$, condition (2.36) for the supersonic upstream flow $\left(\varphi_{e}^{-}, \psi_{e}^{-}\right)$on $\partial_{o} \Omega$ in Theorem 2.3 can be replaced by the $C^{3}$-condition:

$$
\left\|\varphi_{e}^{-}-q_{0}^{-} x_{n}\right\|_{C^{3}}+\left\|\psi_{e}^{-}-q_{0}^{-}\right\|_{C^{2}} \leq \sigma .
$$

This can be achieved by following arguments in Li-Yu [34].

Remark 2.4. There exist $\sigma_{0}$ and $C$ depending only on the data such that equation (2.26) with $Q^{+}$defined by $(2.27)$ has a unique solution $q^{+} \in\left(0, c_{*}\right)$ satisfying $(2.29)$.

This can be seen as follows: From (2.16), it follows that ||$\partial_{o} \Omega|-| \Lambda|| \leq C \sigma$ and $\left|\nu-e_{n}\right| \leq C \sigma$ on $\partial_{o} \Omega$, and hence that $\left|\nu \cdot e_{n}-1\right| \leq C \sigma$ on $\partial_{o} \Omega$. Then

$$
\begin{aligned}
& \left|Q^{+}-\rho\left(\left(q_{0}^{+}\right)^{2}\right) q_{0}^{+}\right| \\
& \quad=\frac{1}{|\Lambda|} \int_{\partial_{o} \Omega}\left|\rho\left(\left|D \varphi^{-}\right|^{2}\right) D \varphi^{-} \cdot \nu-\rho\left(\left(q_{0}^{+}\right)^{2}\right) q_{0}^{+}\right| d x^{\prime}+\left(1-\frac{\left|\partial_{o} \Omega\right|}{|\Lambda|}\right) \rho\left(\left(q_{0}^{+}\right)^{2}\right) q_{0}^{+} \\
& \quad=\frac{1}{|\Lambda|} \int_{\partial_{o} \Omega}\left|\rho\left(\left|D \varphi^{-}\right|^{2}\right) D \varphi^{-} \cdot \nu-\rho\left(\left(q_{0}^{-}\right)^{2}\right) q_{0}^{-}\right| d x^{\prime}+\left(1-\frac{\left|\partial_{o} \Omega\right|}{|\Lambda|}\right) \rho\left(\left(q_{0}^{+}\right)^{2}\right) q_{0}^{+} \\
& \quad \leq \frac{1}{|\Lambda|} \int_{\partial_{o} \Omega}\left(\left|\rho\left(\left|D \varphi^{-}\right|^{2}\right) D \varphi^{-} \cdot \nu-\rho\left(\left|D \varphi_{0}^{-}\right|^{2}\right) D \varphi_{0}^{-} \cdot \nu\right|+\rho\left(\left(q_{0}^{-}\right)^{2}\right) q_{0}^{-}\left|\nu \cdot e_{n}-1\right|\right) d x^{\prime}+C \sigma \\
& \quad \leq C \sigma
\end{aligned}
$$

where we used (2.11) and (2.21). Thus, by (2.6) and (2.7), we obtain that, if $\sigma$ is small depending only on the data, then there exists a unique solution $q^{+} \in\left(0, c_{*}\right)$ of equation $(2.26)$ such that (2.29) holds.

Remark 2.5. In fact, the solutions with transonic shocks of Problem (TN) in Theorem 2.3 are also stable with respect to the nozzle boundaries and the smooth supersonic upstream flows at the entrance; see Theorem 8.1.

Remark 2.6. For the isothermal gas $\gamma=1$, the same results can be obtained by following the same arguments in this paper.

\section{Conormal Problems for Linear Elliptic Equations in a Cylinder}

In this section, we develop a $C^{1, \alpha}$ estimate framework up to the boundary of the cylinder for a conormal problem for linear elliptic equations of divergence form with $C^{\alpha}$ coefficients.

Let $\Lambda \subset \mathbf{R}^{n-1}$ be an open bounded, connected set with $C^{2, \alpha}$ boundary. For $Q>0$, denote

$\mathcal{C}_{Q}:=\Lambda \times(0, Q), \quad \Sigma_{Q}^{1}:=\Lambda \times\left\{x_{n}=0\right\}, \quad \Sigma_{Q}^{2}:=\partial \Lambda \times(0, Q), \quad \Sigma_{Q}^{3}:=\Lambda \times\left\{x_{n}=Q\right\}$.

Consider the problem

$$
\begin{array}{ll}
\sum_{i, j=1}^{n}\left(a_{i j} u_{x_{j}}\right)_{x_{i}}=f & \text { in } \quad \mathcal{C}_{Q}, \\
\sum_{i, j=1}^{n} a_{i j} u_{x_{j}} \nu_{i}=g_{k} & \text { on } \quad \Sigma_{Q}^{k}, k=1,2, \\
u=h \quad \text { on } \quad \Sigma_{Q}^{3}, &
\end{array}
$$

where $\nu$ is the inward unit normal to $\Sigma_{Q}^{1}$ and $\Sigma_{Q}^{2}$ of $\mathcal{C}_{Q}$.

The main issue in the argument below is to show the existence and uniqueness of solutions which are $C^{1, \alpha}$ up to the edge $\partial \Lambda \times\{0\}$ of the cylinder. Lieberman [37] studied general oblique derivative problems for linear elliptic equations in the domains with wedges in which the $C^{1, \beta}$ regularity of solutions is obtained near the wedges. However, these results require certain conditions on the relative geometry of the wedge and the vector fields in the oblique derivative conditions; Moreover, in [37], the Hölder exponent $\beta$ of the solution gradients depends on the geometry of 
wedges and the coefficients of the equation and the right-hand sides of the boundary conditions, and $\beta<\alpha$ in general where $\alpha$ is the Hölder exponent of the coefficients and the right-hand sides. In this section, by restricting the class of equations and considering only conormal boundary conditions and cylindrical domains, we obtain the sharp $C^{1, \alpha}$ regularity of solutions up to $\partial \Lambda \times\{0\}$, i.e., $\beta=\alpha$. This is important in our approach developed in Sections 4-8.

Theorem 3.1. Let $\lambda_{1}, \ldots, \lambda_{n}$, and $\lambda$ are the constants satisfying $0<\lambda \leq \lambda_{i} \leq \lambda^{-1}$ for $i=$ $1, \ldots, n$. Then there exist $\kappa>0$ and $C>0$, depending only on $n, \Lambda, Q$, and $\lambda$, such that, when $a_{i j}(x)$ satisfy

and

$$
\left\|a_{i j}-\lambda_{i} \delta_{j}^{i}\right\|_{0, \alpha, \mathcal{C}_{Q}} \leq \kappa \quad \text { for } i, j=1, \ldots, n,
$$

$$
\begin{array}{cl}
f \in L^{\infty}\left(\mathcal{C}_{Q}\right), & h \in H^{1}\left(\mathcal{C}_{Q}\right), \\
g_{1} \in C^{0, \alpha}\left(\overline{\Sigma_{Q}^{1}}\right), & g_{2} \in C^{0, \alpha}\left(\overline{\Sigma_{Q}^{2}}\right),
\end{array}
$$

there exists a unique weak solution $u \in H^{1}\left(\mathcal{C}_{Q}\right)$ of (3.1)-(3.3) in the sense that $(3.3)$ holds as the trace and

$$
\int_{\mathcal{C}_{Q}}\left(\sum_{i, j=1}^{n} a_{i j} u_{x_{j}} w_{x_{i}}+f w\right) d x+\sum_{k=1}^{2} \int_{\Sigma_{Q}^{k}} g_{k} w d \mathcal{H}^{n-1}=0
$$

for any function $w \in H^{1}\left(\mathcal{C}_{Q}\right)$ with $w=0$ on $\partial \mathcal{C}_{Q} \cap\left\{x_{n}=Q\right\}$. Moreover, the solution $u$ is $C^{1, \alpha}\left(\overline{\mathcal{C}_{Q / 2}}\right)$ and satisfies

$$
\|u\|_{1, \alpha, \mathcal{C}_{Q / 2}} \leq C\left(\|u\|_{L^{2}\left(\mathcal{C}_{Q}\right)}+\|f\|_{0,0, \mathcal{C}_{Q}}+\left\|g_{1}\right\|_{0, \alpha, \Sigma_{Q}^{1}}+\left\|g_{2}\right\|_{0, \alpha, \Sigma_{Q}^{2}}\right) .
$$

Theorem 3.1 is a direct corollary of Theorem 3.2 below, stated in more technical terms. In order to state Theorem 3.2, we introduce some notations and weighted Hölder norms.

Denote

$$
\mathcal{C}_{Q}^{\prime}=\overline{\mathcal{C}_{Q}} \backslash\left\{x_{n}=Q\right\}, \quad\left(\Sigma_{Q}^{2}\right)^{\prime}=\overline{\Sigma_{Q}^{2}} \backslash\left\{x_{n}=Q\right\} .
$$

We will use the following weighted Hölder semi-norms and norms in the cylinder $\mathcal{C}_{Q}$, in which the weight is the distance to the portion of the boundary $\overline{\Sigma_{Q}^{3}}=\partial \mathcal{C}_{Q} \backslash \mathcal{C}_{Q}^{\prime}$. Denote $\delta_{x}=\operatorname{dist}\left(x, \overline{\Sigma_{Q}^{3}}\right)=$ $Q-x_{n}$ for $x=\left(x^{\prime}, x_{n}\right) \in \mathcal{C}_{Q}$ and $\delta_{x, y}=\min \left(\delta_{x}, \delta_{y}\right)$ for $x, y \in \mathcal{C}_{Q}$. For $k \in \mathbf{R}, \alpha \in(0,1)$, and $m \in \mathbf{N}$ (the set of nonnegative integers), define

$$
\begin{aligned}
& {[[u]]_{m ; 0 ; \mathcal{C}_{Q}^{\prime}}^{(k)}=\sum_{|\beta|=m} \sup _{x \in \mathcal{C}_{Q}^{\prime}}\left(\delta_{x}^{m+k}\left|D^{\beta} u(x)\right|\right)} \\
& {[[u]]_{m ; \alpha ; \mathcal{C}_{Q}^{\prime}}^{(k)}=\sum_{|\beta|=m} \sup _{x, y \in \mathcal{C}_{Q}^{\prime}, x \neq y}\left(\delta_{x, y}^{m+\alpha+k} \frac{\left|D^{\beta} u(x)-D^{\beta} u(y)\right|}{|x-y|^{\alpha}}\right)} \\
& |u|_{m ; 0 ; \mathcal{C}_{Q}^{\prime}}^{(k)}=\sum_{j=0}^{m}[[u]]_{j ; 0 ; \mathcal{C}_{Q}^{\prime}}^{(k)} \\
& |u|_{m ; \alpha ; \mathcal{C}_{Q}^{\prime}}^{(k)}=|u|_{m ; 0 ; \mathcal{C}_{Q}^{\prime}}^{(k)}+[[u]]_{m ; \alpha ; \mathcal{C}_{Q}^{\prime}}^{(k)}
\end{aligned}
$$

where $D^{\beta}=\partial_{x_{1}}^{\beta_{1}} \cdots \partial_{x_{n}}^{\beta_{n}}, \beta=\left(\beta_{1}, \ldots, \beta_{n}\right)$ is a multi-index with $\beta_{j} \geq 0, \beta_{j} \in \mathbf{N}$, and $|\beta|=$ $\beta_{1}+\cdots+\beta_{n}$. The weighted Hölder norms $|u|_{m ; \alpha ;\left(\Sigma_{Q}^{2}\right)^{\prime}}^{(k)}$ on the boundary part $\Sigma_{Q}^{2}$ are similarly defined.

Theorem 3.2. Let $\lambda_{1}, \ldots, \lambda_{n}$, and $\lambda$ be the constants satisfying $0<\lambda \leq \lambda_{i} \leq$ lambda $^{-1}$ for $i=1, \ldots, n$. Then there exist $\kappa>0$ and $C>0$, depending only on $n, \Lambda, Q$, and $\lambda$, such that, when $a_{i j}(x)$ satisfy (3.4) and

$$
\begin{gathered}
f \in L^{2}\left(\mathcal{C}_{Q}\right) \cap L_{l o c}^{\infty}\left(\mathcal{C}_{Q}^{\prime}\right), \quad h \in H^{1}\left(\mathcal{C}_{Q}\right), \\
g_{1} \in L^{2}\left(\Sigma_{Q}^{1}\right) \cap C^{0, \alpha}\left(\overline{\Sigma_{Q}^{1}}\right), \quad g_{2} \in L^{2}\left(\Sigma_{Q}^{2}\right) \cap C^{0, \alpha}\left(\left(\Sigma_{Q}^{2}\right)^{\prime}\right)
\end{gathered}
$$


with

$$
|f|_{0,0, \mathcal{C}_{Q}^{\prime}}^{(2+n / 2)},\left|g_{2}\right|_{0, \alpha,\left(\Sigma_{Q}^{2}\right)^{\prime}}^{(1+n / 2)}<\infty
$$

there exists a unique weak solution $u \in H^{1}\left(\mathcal{C}_{Q}\right)$ of (3.1)-(3.3) in the sense that (3.3) holds as the trace and (3.7) holds for any $w \in H^{1}\left(\mathcal{C}_{Q}\right)$ with $w=0$ on $\partial \mathcal{C}_{Q} \cap\left\{x_{n}=Q\right\}$. Moreover, the solution $u$ is $C^{1, \alpha}\left(\mathcal{C}^{\prime}\right)$ and satisfies

$$
|u|_{1, \alpha, \mathcal{C}_{Q}^{\prime}}^{(n / 2)} \leq C\left(\|u\|_{L^{2}\left(\mathcal{C}_{Q}\right)}+|f|_{0,0, \mathcal{C}_{Q}^{\prime}}^{(2+n / 2)}+\left\|g_{1}\right\|_{0, \alpha, \Sigma_{Q}^{1}}+\left|g_{2}\right|_{0, \alpha,\left(\Sigma_{Q}^{2}\right)^{\prime}}^{(1+n / 2)}\right) .
$$

The remaining part of this section is a proof of Theorem 3.2. To prove the existence and regularity part of the theorem, we first consider an auxiliary problem:

$$
\begin{aligned}
& \sum_{i=1}^{n} \lambda_{i} u_{x_{i} x_{i}}=f+\sum_{i=1}^{n} \psi_{x_{i}}^{i} \quad \text { in } \mathcal{C}_{Q}, \\
& \sum_{i=1}^{n} \lambda_{i} u_{x_{i}} \nu_{i} \equiv \lambda_{n} u_{x_{n}}=g_{1} \quad \text { on } \quad \Sigma_{Q}^{1}, \\
& \sum_{i=1}^{n} \lambda_{i} u_{x_{i}} \nu_{i} \equiv \sum_{i=1}^{n-1} \lambda_{i} u_{x_{i}} \nu_{i}=g_{2}+\sum_{i=1}^{n-1} \psi^{i} \nu_{i} \quad \text { on } \Sigma_{Q}^{2}, \\
& u=h \quad \text { on } \Sigma_{Q}^{3},
\end{aligned}
$$

where we used that $\nu=e_{n}$ on $\Sigma_{Q}^{1}$ in (3.15) and $\nu \cdot e_{n}=0$ on $\Sigma_{Q}^{2}$ in (3.16). Note that smooth solutions of (3.14)-(3.17) satisfy

$$
\begin{aligned}
\int_{\mathcal{C}_{Q}}\left(\sum_{i=1}^{n} \lambda_{i} u_{x_{i}} w_{x_{i}}-\sum_{i=1}^{n} \psi^{i} w_{x_{i}}+f w\right) d x \\
\quad+\int_{\Sigma_{Q}^{1}}\left(g_{1}-\psi^{n}\right) w d x^{\prime}-\int_{\Sigma_{Q}^{2}} g_{2} w d \mathcal{H}^{n-1}=0
\end{aligned}
$$

for any function $w \in H^{1}\left(\mathcal{C}_{Q}\right)$ with $w=0$ on $\mathcal{C}_{Q} \cap\left\{x_{n}=Q\right\}$.

Lemma 3.1. Let $\lambda_{1}, \ldots, \lambda_{n}$, and $\lambda$ be the constants satisfying $0<\lambda \leq \lambda_{i} \leq \lambda^{-1}$ for $i=1, \ldots, n$. Let

$$
\begin{aligned}
& f \in L^{2}\left(\mathcal{C}_{Q}\right) \cap L_{\text {loc }}^{\infty}\left(\mathcal{C}_{Q}^{\prime}\right), \quad \psi=\left(\psi^{1}, \ldots, \psi^{n}\right) \in H^{1}\left(\mathcal{C}_{Q}\right) \cap C^{\alpha}\left(\mathcal{C}_{Q}^{\prime}\right), \quad h \in H^{1}\left(\mathcal{C}_{Q}\right), \\
& g_{1} \in L^{2}\left(\Sigma_{Q}^{1}\right) \cap C^{0, \alpha}\left(\overline{\Sigma_{Q}^{1}}\right), \quad g_{2} \in C\left(\mathcal{C}_{Q}^{\prime}\right) \cap L^{2}\left(\Sigma_{Q}^{2}\right) \cap C^{0, \alpha}\left(\mathcal{C}_{Q}^{\prime}\right)
\end{aligned}
$$

with

$$
|f|_{0,0, \mathcal{C}_{Q}^{\prime}}^{(2+n / 2)},|\psi|_{0, \alpha, \mathcal{C}_{Q}^{\prime}}^{(1+n / 2)}, \quad\left|g_{2}\right|_{0, \alpha, \mathcal{C}_{Q}^{\prime}}^{(1+n / 2)}<\infty
$$

Then there exists a unique weak solution $u \in H^{1}\left(\mathcal{C}_{Q}\right)$ of (3.14)-(3.17) in the sense that (3.17) holds as the trace and (3.18) holds for any $w \in H^{1}\left(\mathcal{C}_{Q}\right)$ with $w=0$ on $\partial \mathcal{C}_{Q} \cap\left\{x_{n}=Q\right\}$. The solution $u$ is $C^{1, \alpha}\left(\mathcal{C}^{\prime}\right)$ and satisfies

$$
\begin{aligned}
& \|u\|_{H^{1}\left(\mathcal{C}_{Q}\right)}+|u|_{1, \alpha, \mathcal{C}_{Q}^{\prime}}^{(n / 2)} \\
& \leq C\left(\|f\|_{L^{2}\left(\mathcal{C}_{Q}\right)}+|f|_{0,0, \mathcal{C}_{Q}^{\prime}}^{(2+n / 2)}+\|\psi\|_{L^{2}\left(\mathcal{C}_{Q}\right)}+|\psi|_{0, \alpha, \mathcal{C}_{Q}^{\prime}}^{(1+n / 2)}+\left\|g_{1}\right\|_{0, \alpha, \Sigma_{Q}^{1}}\right. \\
& \left.\quad+\left\|g_{2}\right\|_{L^{2}\left(\Sigma_{Q}^{2}\right)}+\left|g_{2}\right|_{0, \alpha,\left(\Sigma_{Q}^{2}\right)^{\prime}}^{(1+n / 2)}+\|h\|_{H^{1}\left(\mathcal{C}_{Q}\right)}\right)
\end{aligned}
$$

and

$$
|u|_{1, \alpha, \mathcal{C}_{Q}^{\prime}}^{(n / 2)} \leq C\left(\|u\|_{L^{2}\left(\mathcal{C}_{Q}\right)}+|f|_{0,0, \mathcal{C}_{Q}^{\prime}}^{(2+n / 2)}+|\psi|_{0, \alpha, \mathcal{C}_{Q}^{\prime}}^{(1+n / 2)}+\left\|g_{1}\right\|_{0, \alpha, \Sigma_{Q}^{1}}+\left|g_{2}\right|_{0, \alpha,\left(\Sigma_{Q}^{2}\right)^{\prime}}^{(1+n / 2)}\right),
$$

where $C$ depends only on $n, \Lambda, Q$, and $\lambda$. 
Proof. We prove this lemma in six steps. The constant $C$ in the argument below is a universal constant depending only on $n, \Lambda, Q$, and $\lambda$, and may be different at each occurrence.

Step 1. First we show the uniqueness of weak solutions. Suppose $u_{1}$ and $u_{2}$ are two weak solutions of (3.14)-(3.17). Then $u_{1}-u_{2}=0$ on $\Sigma_{Q}^{3}$. Thus, writing the weak forms (3.18) for $u_{1}$ and $u_{2}$ respectively and subtracting the expressions with $w=u_{1}-u_{2}$ yield

$$
0=\int_{\mathcal{C}_{Q}} \sum_{i, j=1}^{n} \lambda_{i}\left|\partial_{x_{i}}\left(u_{1}-u_{2}\right)\right|^{2} d x
$$

Since $u_{1}-u_{2}=0$ on $\Sigma_{Q}^{3}$ and $\lambda_{i}>0$, we conclude $u_{1}=u_{2}$ a.e. in $\mathcal{C}_{Q}$.

Step 2. We now focus on the existence of solutions and estimate (3.21). First we show that the problem can be reduced to the case where the right-hand side of the boundary condition (3.15) vanishes. We can extend $g_{1}$ from $\Sigma_{Q}^{1}=\Lambda \times\{0\}$ to the whole hyperplane $\left\{\left(x^{\prime}, 0\right): x^{\prime} \in \mathbf{R}^{n-1}\right\}$ such that the extension $g_{1}^{*} \in C_{0}^{\alpha}\left(\mathbf{R}^{n-1}\right)$ satisfies $\left\|g_{1}^{*}\right\|_{0, \alpha, \mathbf{R}^{n-1}} \leq C\left\|g_{1}\right\|_{0, \alpha, \Lambda}$. Now, as in [21, page 124-125], we choose a nonnegative $\eta \in C_{0}^{2}\left(\mathbf{R}^{n-1}\right)$ with $\int_{\mathbf{R}^{n-1}} \eta\left(y^{\prime}\right) d y^{\prime}=1$ and define

$$
G\left(x^{\prime}, x_{n}\right)=\lambda_{n}^{-1} x_{n} \int_{\mathbf{R}^{n-1}} g_{1}^{*}\left(x^{\prime}-x_{n} y^{\prime}\right) \eta\left(y^{\prime}\right) d y^{\prime} .
$$

Then $G \in C^{1, \alpha}\left(\overline{\mathbf{R}_{+}^{n}}\right)$ with

$$
\|G\|_{1, \alpha, \mathcal{C}_{Q}} \leq C\left\|g_{1}^{*}\right\|_{0, \alpha, \mathbf{R}^{n-1}} \leq C\left\|g_{1}\right\|_{0, \alpha, \Lambda}
$$

and

$$
\lambda_{n} G_{x_{n}}=g_{1}^{*}=g_{1} \quad \text { on } \Lambda \times\left\{x_{n}=0\right\} .
$$

Thus, $u$ is a weak solution of (3.14)-(3.17) if and only if $v=u-G$ is a weak solution of the same problem with modified right-hand sides: $f, \psi, g_{1}$, and $g_{2}$ are replaced by $\tilde{f}=f, \tilde{\psi}^{i}=\psi^{i}-\lambda_{i} G_{x_{i}}$, $\tilde{g}_{1}=0$, and $\tilde{g}_{2}=g_{2}$. Using (3.23), it is easy to check that (3.21) and (3.22) for $v$ in terms of $\tilde{f}, \tilde{\psi}, \tilde{g}_{1}$, and $\tilde{g}_{2}$ implies (3.21) and (3.22) for $u$ in terms of $f, \psi, g_{1}$, and $g_{2}$.

Thus, from now on, we assume

$$
g_{1}=0 \quad \text { on } \Sigma_{Q}^{1} \text {. }
$$

Step 3. We also note that we can assume without loss of generality that

$$
\psi^{n}=0 \quad \text { on } \Sigma_{Q}^{1} .
$$

Indeed, if $u$ is a weak solution of (3.14)-(3.17), then $u$ is also a weak solution of (3.14)-(3.17) with $\psi^{n}$ replaced by $\hat{\psi}^{n}: \hat{\psi}^{n}\left(x^{\prime}, x_{n}\right)=\psi^{n}\left(x^{\prime}, x_{n}\right)-\psi^{n}\left(x^{\prime}, 0\right)$ for $\left(x^{\prime}, x_{n}\right) \in \mathcal{C}_{Q}$. Since

$$
\left\|\hat{\psi}^{n}\right\|_{L^{2}\left(\mathcal{C}_{Q}\right)}+|\hat{\psi}|_{0, \alpha, \mathcal{C}_{Q}^{\prime}}^{(1+n / 2)} \leq C\left(\left\|\psi^{n}\right\|_{L^{2}\left(\mathcal{C}_{Q}\right)}+|\psi|_{0, \alpha, \mathcal{C}_{Q}^{\prime}}^{(1+n / 2)}\right)
$$

it follows that (3.21) and (3.22) in terms of the right-hand sides with $\psi^{n}$ replaced by $\hat{\psi}^{n}$ implies (3.21) and (3.22) in terms of the original functions.

Clearly, $\hat{\psi}^{n}$ satisfies (3.25). Thus we can assume (3.25) from now on.

Step 4. With (3.24) and (3.25), we extend the problem (3.14)-(3.17) to the cylinder $\mathcal{C}_{(-Q, Q)}=$ $\Lambda \times(-Q, Q)$. Set $\Sigma_{(-Q, Q)}^{2}:=\partial \Lambda \times(-Q, Q)$.

Note that, with (3.24) and (3.25), $u$ is a weak solution of (3.14)-(3.17) if (3.17) holds as the trace and, for any $w \in H^{1}\left(\mathcal{C}_{Q}\right)$ with $w=0$ on $\partial \mathcal{C}_{Q} \cap\left\{x_{n}=Q\right\}$,

$$
\int_{\mathcal{C}_{Q}}\left(\sum_{i=1}^{n} \lambda_{i} u_{x_{i}} w_{x_{i}}-\sum_{i=1}^{n} \psi^{i} w_{x_{i}}+f w\right) d x-\int_{\Sigma_{Q}^{2}} g_{2} w d \mathcal{H}^{n-1}=0 .
$$

We use the even reflection to extend $u, f, h, g_{2}, \psi^{1}, \ldots, \psi^{n-1}$ to $\mathcal{C}_{(-Q, Q)}$ and the odd reflection to extend $\psi^{n}$ to $\mathcal{C}_{(-Q, Q)}$. That is, for $x^{\prime} \in \Lambda$ and $x_{n} \in(0, Q)$, we define, by using the same 
notations for the original and extended functions,

$$
\begin{aligned}
& \varphi\left(x^{\prime},-x_{n}\right)=\varphi\left(x^{\prime}, x_{n}\right) \quad \text { for } \varphi \in\left\{u, f, h, g_{2}, \psi^{1}, \ldots, \psi^{n-1}\right\}, \\
& \psi^{n}\left(x^{\prime},-x_{n}\right)=-\psi^{n}\left(x^{\prime}, x_{n}\right) .
\end{aligned}
$$

Obviously, for $u \in H^{1}\left(\mathcal{C}_{Q}\right)$ and $f, \psi, h$, and $g_{2}$ as in Lemma 3.1 with (3.25) for $\psi^{n}$, the extended functions satisfy

$$
\begin{aligned}
& u \in H^{1}\left(\mathcal{C}_{(-Q, Q)}\right), \quad f \in L^{2}\left(\mathcal{C}_{(-Q, Q)}\right) \cap L_{l o c}^{\infty}\left(\mathcal{C}_{(-Q, Q)}^{\prime}\right), \quad \psi \in H^{1}\left(\mathcal{C}_{(-Q, Q)}\right) \cap C^{\alpha}\left(\mathcal{C}_{(-Q, Q)}^{\prime}\right), \\
& h \in H^{1}\left(\mathcal{C}_{(-Q, Q)}\right), \quad g_{2} \in C^{\alpha}\left(\Sigma_{(-Q, Q)}^{2}\right) \cap L^{2}\left(\Sigma_{(-Q, Q)}^{2}\right),
\end{aligned}
$$

where $\mathcal{C}_{(-Q, Q)}^{\prime}=\mathcal{C}_{(-Q, Q)} \cup \Sigma_{(-Q, Q)}^{2}$. Moreover, defining the weighted Hölder semi-norms and norms $|\cdot|_{m ; \alpha ; \mathcal{C}_{(-Q, Q)}^{\prime}}^{(k)}$ and $|\cdot|_{m ; \alpha ; \Sigma_{(-Q, Q)}^{2}}^{(k)}$ by (3.10) with the supremums taken over the domains $\mathcal{C}_{(-Q, Q)}\left(\right.$ resp. $\left.\Sigma_{(-Q, Q)}^{2}\right)$ and

$$
\delta_{x}=\operatorname{dist}\left(x, \partial \mathcal{C}_{(-Q, Q)} \backslash \Sigma_{(-Q, Q)}^{2}\right)=\min \left(Q-x_{n}, Q+x_{n}\right) \quad \text { for } x=\left(x^{\prime}, x_{n}\right) \in \mathcal{C}_{(-Q, Q)},
$$

we get

$$
\begin{aligned}
& |f|_{0,0, \mathcal{C}_{(-Q, Q)}^{\prime}}^{(2+n / 2)} \leq C|f|_{0,0, \mathcal{C}_{Q}^{\prime}}^{(2+n / 2)}, \quad|\psi|_{0, \alpha, \mathcal{C}_{(-Q, Q)}^{\prime}}^{(1+n / 2)} \leq C|\psi|_{0, \alpha, \mathcal{C}_{Q}^{\prime}}^{(1+n / 2)}, \\
& \left|g_{2}\right|_{0, \alpha, \Sigma_{(-Q, Q)}^{2}}^{(1+n / 2)} \leq C\left|g_{2}\right|_{0, \alpha, \mathcal{C}_{Q}^{\prime}}^{(1+n / 2)},
\end{aligned}
$$

where the norms on the right-hand sides are finite by (3.20).

Then it follows from (3.26) and (3.27) that the extended functions satisfy

$$
\int_{\mathcal{C}_{(-Q, Q)}}\left(\sum_{i=1}^{n} \lambda_{i} u_{x_{i}} w_{x_{i}}-\sum_{i=1}^{n} \psi^{i} w_{x_{i}}+f w\right) d x-\int_{\Sigma_{(-Q, Q)}^{2}} g_{2} w d \mathcal{H}^{n-1}=0
$$

for any $w \in H^{1}\left(\mathcal{C}_{(-Q, Q)}\right)$ satisfying $w=0$ on $\left\{x_{n}= \pm Q\right\}$. Indeed, (3.29) holds separately when the integration is over subdomains $\left\{x_{n}>0\right\}$ and $\left\{x_{n}<0\right\}$ (the last follows by the change of variable $x_{n} \rightarrow-x_{n}$ ). Thus, the extended function $u$ is a weak solution of

$$
\begin{aligned}
& \sum_{i=1}^{n} \lambda_{i} u_{x_{i} x_{i}}=f+\sum_{i=1}^{n} \psi_{x_{i}}^{i} \quad \text { in } \quad \mathcal{C}_{(-Q, Q)}, \\
& \sum_{i=1}^{n} \lambda_{i} u_{x_{i}} \nu_{i} \equiv \sum_{i=1}^{n-1} \lambda_{i} u_{x_{i}} \nu_{i}=g_{2}+\sum_{i=1}^{n-1} \psi^{i} \nu_{i} \quad \text { on } \Sigma_{(-Q, Q)}^{2}, \\
& u=h \quad \text { on } \quad(\Lambda \times\{-Q\}) \cup(\Lambda \times\{Q\}) .
\end{aligned}
$$

Conversely, if $u$ is a weak solution of (3.30)-(3.32) (i.e. (3.29) holds for $w$ as above), then $u$ satisfies $u\left(x^{\prime},-x_{n}\right)=u\left(x^{\prime}, x_{n}\right)$ for $x^{\prime} \in \Lambda$ and $x_{n} \in(0, Q)$. To see this, we first note that a weak solution of (3.29) is unique: The proof similarly follows the proof of uniqueness for (3.1)-(3.3) by using the fact that, if $u_{1}$ and $u_{2}$ are two weak solutions of (3.30)-(3.32), then we can use $w=u_{1}-u_{2}$ in (3.29). Now, the properties in (3.27) for $f, h, \psi$, and $g_{2}$ imply that, if $u\left(x^{\prime}, x_{n}\right)$ is a solution of (3.29), then $u\left(x^{\prime},-x_{n}\right)$ is also a solution. Thus, by uniqueness, $u\left(x^{\prime},-x_{n}\right)=u\left(x^{\prime}, x_{n}\right)$. Now, using the properties (3.27) for $u, f, h, \psi$, and $g_{2}$, we see that, for all the integrals in (3.29), the integrals over subdomains $\left\{x_{n}>0\right\}$ and $\left\{x_{n}<0\right\}$ are equal. Thus, (3.29) implies (3.26). Then, in order to solve (3.14)-(3.17), it suffices to solve (3.30)-(3.32) for the extended right-hand sides.

Step 5. We now derive some estimates for the weak solution $u \in H^{1}\left(\mathcal{C}_{(-Q, Q)}\right)$ of $(3.30)-(3.32)$. We first derive the energy estimate. We can use $w=u-h$ in (3.29) and then use the ellipticity 
and Hölder inequality to obtain

$$
\begin{aligned}
\|u\|_{H^{1}\left(\mathcal{C}_{(-Q, Q)}\right)} \leq \quad C( & \|f\|_{L^{2}\left(\mathcal{C}_{(-Q, Q)}\right)}+\|\psi\|_{L^{2}\left(\mathcal{C}_{(-Q, Q)}\right)} \\
& \left.+\|h\|_{H^{1}\left(\mathcal{C}_{(-Q, Q)}\right)}+\left\|g_{2}\right\|_{L^{2}\left(\Sigma_{(-Q, Q)}^{2}\right)}\right) .
\end{aligned}
$$

Note that, by the standard local regularity results for the conormal problem for the elliptic equations in the domain with $C^{1, \alpha}$ boundary (e.g. [36]), we get $u \in C^{1, \alpha}\left(\mathcal{C}_{(-Q, Q)}^{\prime}\right)$.

Now we derive the estimates for the Hölder norms of $u$. We first recall the standard estimates for the conormal problem for the elliptic equations in a unit ball $B_{1}=B_{1}(0)$ and half-ball $B_{1}^{+}=B_{1}(0) \cap\left\{x_{n}>0\right\}$. If $u \in H^{1}\left(B_{1}\right)$ is a weak solution of the equation

$$
\sum_{i, j=1}^{n}\left(a_{i j} u_{x_{j}}\right)_{x_{i}}=f+\sum_{i=1}^{n} \psi_{x_{i}}^{i} \quad \text { in } B_{1}
$$

where $a_{i j} \in C^{\alpha}\left(B_{1}\right)$ satisfy the ellipticity condition: there exists some $\lambda>0$ such that, for any $x \in B_{1}$,

$$
\lambda|\xi|^{2} \leq \sum_{i, j=1}^{n} a_{i j}(x) \xi_{i} \xi_{j} \leq \lambda^{-1}|\xi|^{2} \quad \text { for any } \quad \xi \in \mathbf{R}^{n}
$$

and, if $f \in L^{\infty}\left(B_{1}\right)$ and $\psi \in C^{\alpha}\left(\overline{B_{1}}\right)$, then $u \in C^{1, \alpha}\left(\overline{B_{1 / 2}}\right)$ with

$$
\|u\|_{1, \alpha, B_{1 / 2}} \leq C\left(\|u\|_{L^{2}\left(B_{1}\right)}+\|f\|_{L^{\infty}\left(B_{1}\right)}+\|\psi\|_{0, \alpha, B_{1}}\right),
$$

where $C$ depends only on $n, \lambda$, and $\left\|a_{i j}\right\|_{0, \alpha, B_{1}}$. This follows from [21, Theorem 8.32], combined with [21, Theorem 8.17]. Similar estimates can be obtained for the conormal problem, in which $u \in H^{1}\left(B_{1}^{+}\right)$is a weak solution of $(3.34)$ in $B_{1}^{+}$with the conormal condition

$$
\sum_{j=1}^{n} a_{n j} u_{x_{j}}=g+\psi^{n} \quad \text { on } \partial^{\prime} B_{1}^{+}:=\partial B_{1}^{+} \cap\left\{x_{n}=0\right\},
$$

where $g \in C^{\alpha}\left(\bar{B}^{+}\right)$. Then $u \in C^{1, \alpha}\left(\overline{B_{1 / 2}^{+}}\right)$with

$$
\|u\|_{1, \alpha, B_{1 / 2}^{+}} \leq C\left(\|u\|_{L^{2}\left(B_{1}^{+}\right)}+\|f\|_{L^{\infty}\left(B_{1}^{+}\right)}+\|\psi\|_{0, \alpha, B_{1}^{+}}+\|g\|_{0, \alpha, \partial^{\prime} B_{1}^{+}}\right),
$$

where $C$ depends on $n, \lambda$, and $\left\|a_{i j}\right\|_{0, \alpha, B_{1}^{+}}$. This can be inferred, e.g., by simplifying the estimates of $[36]$ to the case of linear equations.

Let $\rho \in(0,1 / 2)$. By scaling, we obtain the corresponding local estimates for weak solutions of (3.34) in $B_{\rho}$ and for weak solutions of the conormal problem (3.34) and (3.37) in $B_{\rho}^{+}$. We write only the estimate for the conormal problem in $B_{\rho}^{+}$, since the interior estimate in $B_{\rho}$ differs only in that it does not have the terms with the norms of $g$ on the right-hand side. Thus, if $u$ is a weak solution of (3.34) in $B_{\rho / 2}^{+}$and satisfies (3.37) in $B_{\rho}^{+} \cap\left\{x_{n}=0\right\}$, then $u \in C^{1, \alpha}\left(\overline{B_{\rho / 2}^{+}}\right)$with

$$
\begin{aligned}
& \|u\|_{0,0, B_{\rho / 2}^{+}}+\rho\|D u\|_{0,0, B_{\rho / 2}^{+}}+\rho^{1+\alpha}[D u]_{0, \alpha, B_{\rho / 2}^{+}} \\
& \leq C\left(\rho^{-n / 2}\|u\|_{L^{2}\left(B_{\rho}^{+}\right)}+\rho^{2}\|f\|_{L^{\infty}\left(B_{\rho}^{+}\right)}+\rho\|\psi\|_{0,0, B_{\rho}^{+}}\right. \\
& \left.\quad+\rho^{1+\alpha}[\psi]_{0, \alpha, B_{\rho}^{+}}+\rho\|g\|_{0,0, \partial^{\prime} B_{\rho}^{+}}+\rho^{1+\alpha}[g]_{0, \alpha, \partial^{\prime} B_{\rho}^{+}}\right) .
\end{aligned}
$$

This estimate is obtained by introducing the function $v(x)=u(\rho x)$ in $B_{1}^{+}$, writing the equation and conormal condition satisfied by $u$ in terms of $v$, using estimate (3.38) for $v$ with the corresponding right-hand sides of the equation and conormal condition satisfied by $v$, and then rewriting that estimate in terms of $u$.

Now we get similar estimates for weak solutions of (3.30)-(3.32). Using that $\partial \Lambda$ is $C^{1, \alpha}$ and compact, there exits $r>0$ and $M>0$ such that, for any $z \in \Sigma_{(-Q, Q)}^{2}$ and any positive $\rho<\min \left(r, \delta_{z}\right)$, there is a $C^{1, \alpha}$ diffeomorphism $F$ that flattens $\Sigma_{(-Q, Q)}^{2}$ in $B_{\rho}(z) \cap \mathcal{C}_{(-Q, Q)}$, i.e., $B_{\rho / M}^{+} \subset F\left(B_{\rho}(z) \cap \mathcal{C}_{(-Q, Q)}\right) \subset B_{M \rho}^{+}$, and $\left\|\left(F, F^{-1}\right)\right\|_{C^{1, \alpha}}$ depends only on $\Lambda$. The transformed 
function $v(y):=u\left(F^{-1}(y)\right)$ satisfies a conormal problem of the form (3.34) and (3.37) in $B_{\rho / M}^{+}$, where the equation is elliptic (with ellipticity constant $\lambda / 2$ ) and the $C^{\alpha}$ norms of the coefficients $a_{i j}$ depending only on the $C^{1, \alpha}$ norms of $\partial \Lambda$, and the appropriate norms of right-hand sides of the original and transformed problems are estimated by one in terms of another with a constant depending only on the $C^{1, \alpha}$ norms of $\partial \Lambda$. This can be seen by choosing the function $w$ supported in $B_{\rho}(z)$ in (3.29) and changing variables $x \rightarrow y=F(x)$ in (3.29). Now we get estimate (3.39) for $v$, which implies the following estimate for $u$ :

$$
\begin{aligned}
& \|u\|_{0,0, \Omega_{\rho / 2}(z)}+\rho\|D u\|_{0,0, \Omega_{\rho / 2}(z)}+\rho^{1+\alpha}[D u]_{0, \alpha, \Omega_{\rho / 2}(z)} \\
& \leq C\left(\rho^{-n / 2}\|u\|_{L^{2}\left(\Omega_{\rho}(z)\right)}+\rho^{2}\|f\|_{L^{\infty}\left(\Omega_{\rho}(z)\right)}+\rho\|\psi\|_{0,0, \Omega_{\rho}(z)}+\rho^{1+\alpha}[\psi]_{0, \alpha, \Omega_{\rho}(z)}\right. \\
& \left.\quad+\rho\left\|g_{2}\right\|_{0,0, \partial^{\prime} \Omega_{\rho}(z)}+\rho^{1+\alpha}\left[g_{2}\right]_{0, \alpha, \partial^{\prime} \Omega_{\rho}(z)}\right)
\end{aligned}
$$

for any $z \in \Sigma_{(-Q, Q)}^{2}, 0<\rho<\min \left(r, \delta_{z}\right)$, with $C$ depending only on $n, \Lambda, L$, and $\lambda$, where $\Omega_{\rho}(z):=B_{\rho}(z) \cap \mathcal{C}_{(-Q, Q)}$ and $\partial^{\prime} \Omega_{\rho}(z):=B_{\rho}(z) \cap \Sigma_{(-Q, Q)}^{2}$. We also have the corresponding interior estimates: for any $z \in \mathcal{C}_{(-Q, Q)}$ and $0<\rho<\operatorname{dist}\left(z, \partial \mathcal{C}_{(-Q, Q)}\right)$, we obtain (3.40) for $\Omega_{\rho}(z)=B_{\rho}(z)$ without the terms involving the norms of $g_{2}$ on the right-hand side. Multiplying the interior and boundary estimates (3.40) by $\rho^{n / 2}$ and using a standard argument (e.g., [21, Theorem 4.8]) yields

$$
\begin{aligned}
|u|_{1, \alpha, \mathcal{C}_{(-Q, Q)}^{\prime}}^{(n / 2)} \leq \quad & \left(\|u\|_{L^{2}\left(\mathcal{C}_{(-Q, Q)}\right)}+|f|_{0,0, \mathcal{C}_{(-Q, Q)}^{\prime}}^{(2+n / 2)}\right. \\
& \left.+|\psi|_{0, \alpha, \mathcal{C}_{(-Q, Q)}^{\prime}}^{(1+n / 2)}+\left|g_{2}\right|_{0, \alpha, \Sigma_{(-Q, Q)}^{2}}^{(1+n / 2)}\right) .
\end{aligned}
$$

Obviously, estimates (3.33) and (3.41) imply (3.21). Also, (3.41) implies (3.22).

Step 6. It remains to prove the existence of a weak solution $u \in H^{1}\left(\mathcal{C}_{(-Q, Q)}\right)$ of $(3.30)-(3.32)$. We first assume that $f, \psi \in C^{\infty}\left(\overline{\mathcal{C}_{(-Q, Q)}}\right)$ and $g_{2} \in C^{\infty}\left(\overline{\Sigma_{(-Q, Q)}^{2}}\right)$. Then, since $\Sigma_{(-Q, Q)}^{2}$ is a $C^{2, \alpha}$ surface and $g_{2}+\sum_{i=1}^{n-1} \psi^{i} \nu_{i} \in C^{1, \alpha}\left(\overline{\Sigma_{(-Q, Q)}^{2}}\right)$, we can find $G \in C^{2, \alpha}\left(\mathcal{C}_{(-Q, Q)}^{\prime}\right)$ such that

$$
\sum_{i=1}^{n-1} \lambda_{i} G_{x_{i}} \nu_{i}=g_{2}+\sum_{i=1}^{n-1} \psi^{i} \nu_{i} \quad \text { on } \quad \Sigma_{(-Q, Q)}^{2} .
$$

To construct such a function $G$, we first extend the right-hand side of $(3.42)$ to $\Sigma_{(-2 Q, 2 Q)}^{2}=$ $\partial \Lambda \times(-2 Q, 2 Q)$ so that $\tilde{g} \in C^{1, \alpha}\left(\overline{\Sigma_{(-2 Q, 2 Q)}^{2}}\right)$ with $\tilde{g}=g_{2}+\sum_{i=1}^{n-1} \psi^{i} \nu_{i}$ on $\Sigma_{(-Q, Q)}^{2}$. Then, for every $x_{*} \in \partial \Lambda \times(-3 Q / 2,3 Q / 2)$, we can locally flatten $\Sigma_{(-2 Q, 2 Q)}^{2}$ by a $C^{2, \alpha}$ diffeomorphism $\Psi: \mathbf{R}^{n} \rightarrow \mathbf{R}^{n}$, i.e., for some $r>0, \Psi\left(B_{r}\left(x_{*}\right) \cap(\Lambda \times \mathbf{R})\right)=B_{1}(0) \cap\left\{x_{n}>0\right\}$ with $\Psi\left(B_{r}\left(x_{*}\right) \cap\right.$ $(\partial \Lambda \times \mathbf{R}))=B_{1}(0) \cap\left\{x_{n}=0\right\}$. Then condition (3.42) is transformed into

$$
\sum_{i=1}^{n} c_{i}\left(x^{\prime}\right) \hat{G}_{x_{i}}=\hat{g} \quad \text { on } \quad\left\{x_{n}=0\right\}
$$

where $c_{i} \in C^{1, \alpha}\left(\mathbf{R}^{n-1}\right)$ with $c_{n} \geq \min \left(\lambda_{1}, \ldots, \lambda_{n}\right) / 2>0$ if $r$ is chosen sufficiently small, and $\hat{g} \in$ $C^{1, \alpha}\left(\mathbf{R}^{n-1}\right)$ with compact support is obtained by transforming $\tilde{g}$ by $\Psi$ and extending to $\mathbf{R}^{n-1}$. Now, by the argument similar to [21, page 124-125], we choose a nonnegative $\eta \in C_{0}^{2}\left(\mathbf{R}^{n-1}\right)$ with $\int_{\mathbf{R}^{n-1}} \eta\left(y^{\prime}\right) d y^{\prime}=1$, define the function

$$
\hat{G}\left(x^{\prime}, x_{n}\right)=x_{n} \int_{\mathbf{R}^{n-1}} \frac{\hat{g}\left(x^{\prime}-x_{n} y^{\prime}\right)}{c_{n}\left(x^{\prime}-x_{n} y^{\prime}\right)} \eta\left(y^{\prime}\right) d y^{\prime},
$$

and show that $\hat{G}$ is $C^{2, \alpha}\left(\overline{\mathbf{R}_{+}^{n}}\right)$ and satisfies (3.43). Transforming $\hat{G}$ back by $\Psi^{-1}$, we obtain $G \equiv G^{x_{*}} \in C^{2, \alpha}\left((\bar{\Lambda} \times \mathbf{R}) \cap B_{r}\left(x_{*}\right)\right)$ satisfying $(3.42)$ on $\Sigma_{(-2 Q, 2 Q)}^{2} \cap B_{r}\left(x_{*}\right)$. Gluing these local functions by using a partition of unity, we get a $C^{2, \alpha}$ function $G$ in a neighborhood of the 
boundary part $\Sigma_{(-Q, Q)}^{2}$ in $\mathcal{C}$, which satisfies $(3.42)$ on $\Sigma_{(-Q, Q)}^{2}$. Finally, we extend $G$ to the whole cylinder $\mathcal{C}_{(-Q, Q)}$ with $G \in C^{2, \alpha}\left(\mathcal{C}_{(-Q, Q)}^{\prime}\right)$.

Now, denoting $\tilde{f}=f+\sum_{i=1}^{n}\left(\psi_{x_{i}}^{i}-\lambda_{i} G_{x_{i} x_{i}}\right)$, we have $\tilde{f} \in C\left(\overline{\mathcal{C}_{(-Q, Q)}}\right)$. Thus we can solve the variational problem of minimizing

$$
I[v]=\int_{\mathcal{C}_{(-Q, Q)}}\left(\frac{1}{2} \sum_{i=1}^{n} \lambda_{i} v_{x_{i}}^{2}+\tilde{f} v\right) d x
$$

on the set $\left\{v \in H^{1}\left(\mathcal{C}_{(-Q, Q)}\right): v=h-G\right.$ on $\left.\Lambda \times\left\{x_{n}= \pm Q\right\}\right\}$. If $v$ is a minimizer, then $u=v+G$ is a weak solution of (3.30)-(3.32).

For general $f, \psi \in L^{2}\left(\mathcal{C}_{(-Q, Q)}\right)$ and $g_{2} \in L^{2}\left(\Sigma_{(-Q, Q)}^{2}\right)$, we approximate by $f_{l}, \psi_{l} \in C^{\infty}\left(\overline{\mathcal{C}_{(-Q, Q)}}\right)$ and $g_{2}^{l} \in C^{\infty}\left(\overline{\Sigma_{(-Q, Q)}^{2}}\right)$ such that $\left(f_{l}, \psi_{l}\right) \rightarrow(f, \psi)$ in $L^{2}\left(\mathcal{C}_{(-Q, Q)}\right)$ and $g_{2}^{l} \rightarrow g_{2}$ in $L^{2}\left(\Sigma_{(-Q, Q)}^{2}\right)$. Then, for each $l$, we can find a weak solution $u_{l} \in H^{1}\left(\mathcal{C}_{(-Q, Q)}\right)$ of $(3.30)-(3.32)$ with functions $f_{l}, \psi_{l}$, and $g_{2}^{l}$ on the right-hand side. Since $u_{k}-u_{l}$ satisfies (3.30)-(3.32) with $f_{k}-f_{l}, \psi_{k}-\psi_{l}$, $g_{2}^{k}-g_{2}^{l}$, and $h=0$ on the right-hand side, we apply (3.33) to see that $\left\{u_{l}\right\}$ is a Cauchy sequence in $H^{1}\left(\mathcal{C}_{(-Q, Q)}\right)$ and its limit $u$ is a solution of (3.30)-(3.32) with the original right-hand sides. Now Lemma 3.1 is proved.

Proof of Theorem 3.2. If $\kappa$ is small depending on $\lambda$, then (3.4) implies the ellipticity (3.35) with $\frac{3}{4} \lambda$ instead of $\lambda$. We choose such $\kappa$ below.

First we show the uniqueness of weak solutions. Suppose $u_{1}$ and $u_{2}$ are two weak solutions of (3.1)-(3.3). Then $u_{1}-u_{2}=0$ on $\Sigma_{Q}^{3}$. Writing the weak form (3.7) for $u_{1}$ and $u_{2}$ respectively, subtracting the expressions with $w=u_{1}-u_{2}$, and then using the ellipticity yields

$$
0=\int_{\mathcal{C}_{Q}} \sum_{i, j=1}^{n} a_{i j} \partial_{x_{i}}\left(u_{1}-u_{2}\right) \partial_{x_{j}}\left(u_{1}-u_{2}\right) d x \geq \frac{3}{4} \lambda \int_{\mathcal{C}_{Q}}\left|D\left(u_{1}-u_{2}\right)\right|^{2} d x .
$$

Since $u_{1}-u_{2}=0$ on $\Sigma_{Q}^{3}$, we conclude $u_{1}=u_{2}$ a.e. in $\mathcal{C}_{Q}$.

We now apply the Banach contraction fixed point theorem to prove the existence and regularity of the weak solution $u \in H^{1}\left(\mathcal{C}_{Q}\right)$ of $(3.1)-(3.3)$ for sufficiently small $\kappa>0$ in the space:

$$
\mathcal{K}=\left\{v \in H^{1}\left(\mathcal{C}_{Q}\right) \cap C^{1, \alpha}\left(\mathcal{C}_{Q}^{\prime}\right):\|v\|_{\mathcal{K}}:=\|v\|_{H^{1}\left(\mathcal{C}_{Q}\right)}+|v|_{1, \alpha, \mathcal{C}_{Q}^{\prime}}^{(n / 2)}<\infty\right\} .
$$

We now define a mapping $J: \mathcal{K} \rightarrow \mathcal{K}$. For $v \in \mathcal{K}$, consider the problem (3.14)-(3.17) with functions $\tilde{f}, \tilde{h}, \tilde{g}_{1}, \tilde{g}_{2}$, and $\tilde{\psi}$ on the right-hand sides, where

$$
\begin{aligned}
& \tilde{f}=f, \quad \tilde{h}=h, \quad \tilde{g}_{2}=g_{2}, \\
& \tilde{g}_{1}(x)=g_{1}(x)+\sum_{i, j=1}^{n}\left(\lambda_{i} \delta_{i}^{j}-a_{i j}(x)\right) v_{x_{j}}(x) \nu_{i}(x) \quad \text { for } x \in \Sigma_{Q}^{1}=\Lambda \times\{0\}, \\
& \tilde{\psi}^{i}(x)=\sum_{j=1}^{n}\left(\lambda_{i} \delta_{i}^{j}-a_{i j}(x)\right) v_{x_{j}}(x) \quad \text { for } x \in \mathcal{C}_{Q}, \quad i=1, \ldots, n,
\end{aligned}
$$

where $f, g_{1}, g_{2}$, and $h$ are from (3.1)-(3.3). Since $\nu=e_{n}$ on $\Sigma_{Q}^{1}$, we have

$$
\tilde{g}_{1}=g_{1}+\sum_{j=1}^{n}\left(\lambda_{n} \delta_{n}^{j}-a_{n j}\right) v_{x_{j}} \equiv g_{1}+\tilde{\psi}^{n} \quad \text { on } \quad x \in \Sigma_{Q}^{1} .
$$

By (3.4) and $v \in \mathcal{K}$, the functions $\tilde{g}_{1}$ and $\psi$ satisfy the conditions of Lemma 3.1. Thus, by Lemma 3.1 , there exists a unique weak solution $u$ of (3.14)-(3.17) with the right-hand sides described above which satisfies (3.21) and thus implies $u \in \mathcal{K}$. We define the mapping $J: \mathcal{K} \rightarrow \mathcal{K}$ by setting $J v=u$. 
Now we show that $J$ is a contraction mapping in the norm $\|\cdot\|_{\mathcal{K}}$ if $\kappa>0$ is small. Let $v^{1}, v^{2} \in \mathcal{K}$ and $u^{k}=J v^{k}$ for $k=1,2$. Then $u^{1}-u^{2}$ is a weak solution of (3.14)-(3.17) with the following functions $\hat{f}, \hat{h}, \hat{g}_{1}, \hat{g}_{2}$, and $\hat{\psi}$ on the right-hand sides:

$$
\begin{aligned}
& \hat{f}=0, \quad \hat{g}_{2}=0, \quad \hat{h}=0, \\
& \hat{\psi}^{i}(x)=\sum_{j=1}^{n}\left(\lambda_{i} \delta_{i}^{j}-a_{i j}(x)\right)\left(v_{x_{j}}^{1}(x)-v_{x_{j}}^{2}(x)\right) \quad \text { for } x \in \mathcal{C}_{Q}, \quad i=1, \ldots, n, \\
& \hat{g}_{1}(x)=\sum_{j=1}^{n}\left(\lambda_{n} \delta_{n}^{j}-a_{n j}(x)\right)\left(v_{x_{j}}^{1}(x)-v_{x_{j}}^{2}(x)\right) \quad \text { for } x \in \Sigma_{Q}^{1} .
\end{aligned}
$$

By (3.4), we get

$$
\begin{aligned}
\|\hat{\psi}\|_{L^{2}\left(\mathcal{C}_{Q}\right)} & \leq C \kappa\left\|D v^{1}-D v^{2}\right\|_{L^{2}\left(\mathcal{C}_{Q}\right)} \leq C \kappa\left\|v^{1}-v^{2}\right\|_{\mathcal{K}} \\
|\hat{\psi}|_{0, \alpha, \mathcal{C}_{Q}^{\prime}}^{(1+n / 2)} & \leq C \kappa\left|D v^{1}-D v^{2}\right|_{0, \alpha, \mathcal{C}_{Q}^{\prime}}^{(1+n / 2)} \leq C \kappa\left|v^{1}-v^{2}\right|_{1, \alpha, \mathcal{C}_{Q}^{\prime}}^{(n / 2)} \leq C \kappa\left\|v^{1}-v^{2}\right\|_{\mathcal{K}} \\
\left\|\hat{g}_{1}\right\|_{0, \alpha, \Sigma_{Q}^{1}} & \leq C \kappa\left\|D v^{1}-D v^{2}\right\|_{0, \alpha, \Sigma_{Q}^{1}} \leq C \kappa\left|D v^{1}-D v^{2}\right|_{0, \alpha, \mathcal{C}_{Q}^{\prime}}^{(1+n / 2)} \\
& \leq C \kappa\left|v^{1}-v^{2}\right|_{1, \alpha, \mathcal{C}_{Q}^{\prime}}^{(n / 2)} \leq C \kappa\left\|v^{1}-v^{2}\right\|_{\mathcal{K}} .
\end{aligned}
$$

Thus, from (3.21),

$$
\left\|u^{1}-u^{2}\right\|_{\mathcal{K}} \leq C \kappa\left\|v^{1}-v^{2}\right\|_{\mathcal{K}}
$$

Therefore, the mapping $J: \mathcal{K} \rightarrow \mathcal{K}$ is a contraction mapping in the norm $\|\cdot\|_{\mathcal{K}}$ if $\kappa<1 / C$, i.e., $\kappa$ is small depending only on $n, \Lambda, Q$, and $\lambda$.

For such $\kappa$, there exists a fixed point $u \in \mathcal{K}$ satisfying $J u=u$. Then $u$ is a weak solution of (3.1)-(3.3). Indeed, since $J u=u$, then $u$ satisfies (3.18) with right-hand sides given by (3.44), (3.45), and (3.46) computed with $v=u$. Rearranging and taking into account the last expression in (3.47) yields (3.7). Also, since $u$ satisfies (3.18) with right-hand sides given by (3.44), (3.45), and (3.46), $u$ satisfies (3.22) with these right-hand sides, which implies, by estimating $\tilde{\psi}$ and $\tilde{g}_{1}$ similar to the estimates of $\hat{\psi}$ and $\hat{g}_{1}$ above and with $v=u$ :

$$
|u|_{1, \alpha, \mathcal{C}_{Q}^{\prime}}^{(n / 2)} \leq C\left(\|u\|_{L^{2}\left(\mathcal{C}_{Q}\right)}+|f|_{0,0, \mathcal{C}_{Q}^{\prime}}^{(2+n / 2)}+\left\|g_{1}\right\|_{0, \alpha, \Sigma_{Q}^{1}}+\left|g_{2}\right|_{0, \alpha,\left(\Sigma_{Q}^{2}\right)^{\prime}}^{(1+n / 2)}\right)+C \kappa|u|_{1, \alpha, \mathcal{C}_{Q}^{\prime}}^{(n / 2)} .
$$

If $\kappa$ is small, this implies (3.13). Theorem 3.2 is proved.

\section{Free boundary problems in the infinite NOZZLE}

In order to solve Problem (TN), we first reformulate it into a free boundary problem for the subsonic part of the solution, since Problem (TN) is originally hyperbolic-elliptic mixed.

We first modify equation (1.1) to make it uniformly elliptic so that it coincides with the original equation in the range $D \varphi$ in the subsonic region $\Omega^{+}$for $\varphi$ satisfying (2.31) with sufficiently small $\sigma$. The details of the truncation procedure are in [4, Section 4.2].

Let $\varepsilon=\left(c_{*}-q_{0}^{+}\right) / 2$. Then there exists $\tilde{\rho} \in C^{1,1}([0, \infty))$ and $c_{j}>0, j=1,2,3$, depending only on $q_{0}^{+}$and $\gamma$, such that

$$
\begin{aligned}
& \tilde{\rho}\left(q^{2}\right)=\rho\left(q^{2}\right) \quad \text { if } \quad 0 \leq q<c_{*}-\varepsilon \\
& \tilde{\rho}\left(q^{2}\right)=c_{0}+\frac{c_{1}}{q} \quad \text { if } \quad q>c_{*}-\varepsilon \\
& 0<c_{0} \leq\left(\tilde{\rho}\left(q^{2}\right) q\right)^{\prime} \leq c_{2} \quad \text { for } \quad q \in(0, \infty) .
\end{aligned}
$$

Then the equation

$$
\tilde{\mathcal{L}} \varphi:=\operatorname{div}\left(\tilde{\rho}\left(|D \varphi|^{2}\right) D \varphi\right)=0
$$

is uniformly elliptic, whose ellipticity constants depend only on $q_{0}^{+}$and $\gamma$. 
To formulate the problem into a free boundary problem, the main point is to replace the pointwise gradient condition $\left\{|D \varphi(x)|<c_{*}\right\}$ defining $\Omega^{+}(\varphi)$ by a condition stated in terms of $\varphi$ so that our problem is formulated into the framework of free boundary problems.

To do that, we first need to construct a local $C^{1, \alpha}$ supersonic solution $\varphi^{-}$in the domain $\Omega_{1}:=\Omega \cap\left\{-1<x_{n}<1\right\}$ of the initial-boundary value problem (2.18)-(2.19) for the nonlinear hyperbolic equation (1.1) such that

$$
\left\|\varphi^{-}-\varphi_{0}^{-}\right\|_{1, \alpha, \Omega_{1}} \leq C_{1} \sigma
$$

when $\sigma$ is sufficiently small. This is ensured by the local existence theorem, Proposition A.1 in Appendix. By the standard extension argument (see [5] and Section 7.2), we can extend $\varphi^{-}$to the whole infinite nozzle $\Omega$ such that

$$
\left\|\varphi^{-}-\varphi_{0}^{-}\right\|_{1, \alpha, \Omega} \leq C_{0} \sigma,\left.\quad \varphi_{\nu}^{-}\right|_{\partial_{l} \Omega}=0
$$

Then the following heuristic observation motivates our formulation: By $(2.13)$ and $q_{0}^{-}>q_{0}^{+}$, we have $\Omega^{+}\left(\varphi_{0}\right)=\left\{x \in \Omega: \varphi_{0}(x)<\varphi_{0}^{-}(x)\right\}$. Since $\varphi^{-}$is a small $C^{1, \alpha}$ perturbation of $\varphi_{0}^{-}$ and $q_{0}^{-}>q_{0}^{+}$, then we expect that $\varphi^{+}$is close to $\varphi_{0}^{+}$in $C^{1, \alpha}\left(\overline{\Omega^{+}(\varphi)}\right)$ so that we can expect that $\Omega^{+}(\varphi)=\left\{x \in \Omega: \varphi(x)<\varphi^{-}(x)\right\}$.

We also perform the corresponding truncation of the free boundary condition (2.4):

$$
\tilde{\rho}\left(|D \varphi|^{2}\right) \varphi_{\nu}=\rho\left(\left|D \varphi^{-}\right|^{2}\right) D \varphi^{-} \cdot \nu \quad \text { on } S .
$$

On the right-hand side of (4.7), we use the original function $\rho$ since $\rho \neq \tilde{\rho}$ on the range of $\left|D \varphi^{-}\right|^{2}$. Note that (4.7), with the right-hand side considered as a known function, is the conormal boundary condition for the uniformly elliptic equation (4.4).

We first solve the following free boundary problem (TFB), which is a truncated version of Problem $(\mathrm{FB})$, in which the gradient condition that determines $\Omega^{+}$in Problem (TN) is also replaced by the condition $\varphi \leq \varphi^{-}$in $\Omega$ with the definition $\Omega^{+}:=\left\{\varphi<\varphi^{-}\right\} \cap \Omega$.

Problem (TFB): Truncated Free Boundary Problem. Given a supersonic upstream flow $\varphi^{-} \in C^{1, \alpha}\left(\Omega_{1}\right)$ of (1.1) satisfying (4.5), (2.18), and (2.19) for small $\sigma>0$ and some constant $C_{0}$, find $\varphi \in C(\Omega)$ such that

(i) $\varphi$ satisfies

$$
\varphi \leq \varphi^{-} \quad \text { in } \Omega
$$

and conditions (2.18) and (2.19) on the boundary and (2.20) at infinity;

(ii) $\varphi \in C^{1, \alpha}\left(\overline{\Omega^{+}}\right) \cap C^{2}\left(\Omega^{+}\right)$is a solution of $(4.4)$ in $\Omega^{+}:=\left\{\varphi<\varphi^{-}\right\} \cap \Omega$, the non-coincidence set;

(iii) the free boundary $S=\partial \Omega^{+} \cap \Omega$ is given by the equation $x_{n}=f\left(x^{\prime}\right)$ so that $\Omega^{+}=\left\{x_{n}>f\left(x^{\prime}\right)\right\} \cap \Omega$ with $f \in C^{1, \alpha}$

(iv) the free boundary condition (4.7) holds on $S$.

In this paper we develop the iteration approach based on [4, 5], uniform estimate techniques at infinity, and the regularity estimate techniques near the nonsmooth boundary to construct a unique solution of Problem (TFB). Finally, we use an estimate for $|D \varphi|$ to conclude that the solution of the truncated problem, Problem (TFB), is actually a solution of Problem (FB) and thus Problem (TN).

\section{Iteration Procedure And Uniform Estimates}

We now introduce an iteration procedure to construct approximate solutions of Problem (TFB) in the domain $\Omega$ and make uniform estimates of the solutions on the unbounded domain with nonsmooth boundary. 
5.1. Iteration Set. Let $M \geq 1$. We set

$$
\mathcal{K}_{M}:=\left\{\psi \in C^{1, \alpha}(\bar{\Omega}):\left\|\psi-q^{+} x_{n}\right\|_{1, \alpha, \Omega} \leq M \sigma\right\},
$$

where $q^{+}$is defined by equation (2.26) (see Remark 2.4) and $\alpha \in(0,1)$. From the definition, $\mathcal{K}_{M}$ is convex. Now we will show that $\mathcal{K}_{M}$ is compact in the weighted Hölder space $C_{(-1)}^{1, \alpha / 2}(\bar{\Omega})$ as defined below.

For $\alpha \in(0,1), m \in \mathbf{N}$ (the set of nonnegative integers), and $k \in \mathbf{R}$, the definition of the spaces $C_{(k)}^{m, \alpha}(\bar{\Omega})$ is

$$
C_{(k)}^{m, \alpha}(\bar{\Omega}):=\left\{u \in C^{m, \alpha}(\bar{\Omega}) \quad: \quad\|u\|_{m, \alpha, \Omega}^{(k)}<\infty\right\},
$$

where $\|u\|_{m ; \alpha ; \Omega}^{(k)}=\|u\|_{m ; 0 ; \Omega}^{(k)}+[u]_{m ; \alpha ; \Omega}^{(k)}$ with

$$
\begin{aligned}
& \|u\|_{m ; 0 ; \Omega}^{(k)}=\sum_{0 \leq j \leq m}\left(\sum_{|\beta|=j} \sup _{x \in \Omega}\left(\delta_{x}^{k}\left|D^{\beta} u(x)\right|\right)\right), \\
& {[u]_{m ; \alpha ; \Omega}^{(k)}=\sum_{|\beta|=m} \sup _{x, y \in \Omega, x \neq y}\left(\delta_{x, y}^{k} \frac{\left|D^{\beta} u(x)-D^{\beta} u(y)\right|}{|x-y|^{\alpha}}\right),}
\end{aligned}
$$

where $\delta_{x}=\left|x_{n}\right|+1$ for $x=\left(x^{\prime}, x_{n}\right) \in \mathcal{D}$ and $\delta_{x, y}=\min \left(\delta_{x}, \delta_{y}\right)$ for $x, y \in \mathcal{D}$.

Lemma 5.1. If $\alpha>\beta>0$, then $\mathcal{K}_{M}$ is a compact subset of $C_{(-1)}^{1, \beta}(\bar{\Omega})$.

Proof. Let $\psi_{j} \in \mathcal{K}_{M}$ for $j=1,2, \ldots$ By a standard argument, we can extract a subsequence (still denoted by) $\psi_{j}$, which converges in $C^{1, \beta}$ on every compact subset of $\bar{\Omega}$ to the limit $\psi \in C^{1, \alpha}(\bar{\Omega})$. Then $\left\|\psi-q^{+} x_{n}\right\|_{1, \alpha, \Omega} \leq M \sigma$ and thus $\psi \in \mathcal{K}_{M}$. It remains to show that $\left\|\psi_{j}-\psi\right\|_{1, \beta, \Omega}^{(-1)} \rightarrow 0$ as $j \rightarrow \infty$.

Fix $0<\varepsilon<1$. Then $\left\|\psi_{j}\right\|_{1, \alpha, \Omega \cap\left\{x_{n}>1 / \varepsilon\right\}}^{(-1)} \leq\left(M \sigma+q^{+}\right) \varepsilon$, and the same estimate holds for $\psi$. Also, since $\psi_{j} \rightarrow \psi$ in $C^{1, \beta}\left(\bar{\Omega} \cap\left\{x_{n} \leq 2 / \varepsilon\right\}\right)$, there exists $j_{0}$ such that, for $j>j_{0}$, $\left\|\psi_{j}-\psi\right\|_{1, \beta, \Omega \cap\left\{x_{n} \leq 2 / \varepsilon\right\}}^{(-1)} \leq \varepsilon$. Then, for $j>j_{0}$, we have $\left\|\psi_{j}-\psi\right\|_{1, \beta, \Omega}^{(-1)} \leq C \varepsilon$, and the assertion is proved.

5.2. Construction of the Iteration Scheme. Let $\psi \in \mathcal{K}_{M}$. Since $q_{0}^{-}>q_{0}^{+}$, it follows that, if

$$
\sigma \leq \frac{q_{0}^{-}-q_{0}^{+}}{C(M+1)}
$$

with large $C$ depending only on $n$, then (4.5) implies

$$
\left(\varphi^{-}-\psi\right)_{x_{n}}(x) \geq \frac{q_{0}^{-}-q_{0}^{+}}{2}>0 \text { in } \Omega
$$

and $\varphi^{-}>\psi$ on $\left\{x_{n}>1\right\}$. Then the set $\Omega^{+}(\psi):=\left\{\psi<\varphi^{-}\right\} \cap \Omega$ has the form:

$$
\Omega^{+}(\psi)=\left\{x_{n}>f\left(x^{\prime}\right)\right\} \cap \Omega, \quad\|f\|_{1, \alpha, \mathbf{R}^{n-1}} \leq C M \sigma
$$

with $C$ depending only on $q_{0}^{-}-q_{0}^{+}$.

The inward unit normal to $S_{\psi}:=\left\{x_{n}=f\left(x^{\prime}\right)\right\} \cap \Omega$ of $\Omega^{+}$is

$$
\nu_{\psi}(x)=\frac{D \varphi^{-}(x)-D \psi(x)}{\left|D \varphi^{-}(x)-D \psi(x)\right|} \quad \text { for } x \in S_{\psi} .
$$

By the definition of $\mathcal{K}_{M}$ and (5.3), formula (5.6) also defines $\nu_{\psi}(x)$ on $\Omega_{1}$ and

$$
\left\|\nu_{\psi}-e_{n}\right\|_{0, \alpha, \Omega_{1}} \leq C M \sigma \quad \text { with } C=C\left(q_{0}^{+}, q_{0}^{-}\right) .
$$

Motivated by (4.7), we define the function

$$
G_{\psi}(x):=\rho\left(\left|D \varphi^{-}(x)\right|^{2}\right) D \varphi^{-}(x) \cdot \nu_{\psi}(x) \quad \text { on } \Omega_{1} .
$$


Then we consider the following elliptic problem in the domain $\Omega^{+}(\psi)$ :

$$
\begin{aligned}
& \operatorname{div}\left(\tilde{\rho}\left(|D \varphi|^{2}\right) D \varphi\right)=0 \quad \text { in } \quad \Omega^{+}(\psi), \\
& \tilde{\rho}\left(|D \varphi|^{2}\right) \varphi_{\nu}=G_{\psi}(x) \quad \text { on } \quad S_{\psi}:=\left\{x_{n}=f\left(x^{\prime}\right)\right\}, \\
& \varphi_{\nu}=0 \quad \text { on } \partial_{l} \Omega^{+}:=\partial \Omega^{+}(\psi) \cap \partial_{l} \Omega, \\
& \lim _{R \rightarrow \infty}\left\|\varphi-q^{+} x_{n}\right\|_{L^{\infty}\left(\Omega^{+}(\psi) \cap\left\{x_{n}>R\right\}\right)}=0,
\end{aligned}
$$

show that there exists a unique solution $\varphi(x)$, and extend the solution to the whole domain $\Omega$ so that $\varphi \in \mathcal{K}_{M}$.

We rewrite problem (5.9)-(5.12) in terms of function $u(x)=\varphi(x)-q^{+} x_{n}$. We first note that the boundary condition (5.11) is equivalent to

$$
\tilde{\rho}\left(|D \varphi|^{2}\right) \varphi_{\nu}=0 \quad \text { on } \quad \partial_{l} \Omega^{+}
$$

Now $u(x)$ is a solution of the following problem:

$$
\begin{aligned}
& \operatorname{div} A(D u)=0 \quad \text { in } \quad \Omega^{+}(\psi), \\
& A(D u) \cdot \nu=g_{\psi}(x) \quad \text { on } \quad S_{\psi}, \\
& A(D u) \cdot \nu=-\tilde{\rho}\left(\left(q^{+}\right)^{2}\right) q^{+} \nu \cdot e_{n} \quad \text { on } \quad \partial_{l} \Omega^{+}, \\
& \lim _{R \rightarrow \infty}\|u\|_{L^{\infty}\left(\Omega^{+}(\psi) \cap\left\{x_{n}>R\right\}\right)}=0,
\end{aligned}
$$

where

$$
\begin{aligned}
& A(P)=\tilde{\rho}\left(\left|P+q^{+} e_{n}\right|^{2}\right)\left(P+q^{+} e_{n}\right)-\tilde{\rho}\left(\left(q^{+}\right)^{2}\right) q^{+} e_{n} \quad \text { for } P \in \mathbf{R}^{n}, \\
& g_{\psi}(x)=G_{\psi}(x)-\rho\left(\left(q^{+}\right)^{2}\right) q^{+} \nu \cdot e_{n} .
\end{aligned}
$$

Thus, $u(x)$ satisfies the uniformly elliptic equation with the same ellipticity constants as in (4.4):

$$
\lambda|\xi|^{2} \leq \sum_{i, j=1}^{n} A_{P_{j}}^{i}(P) \xi_{i} \xi_{j} \leq \lambda^{-1}|\xi|^{2} \quad \text { for any } \quad P, \xi \in \mathbf{R}^{n} .
$$

Moreover, from the definition of $A(P),(4.1)$, and (4.2), $A(P)$ satisfies

$$
A(0)=0, \quad(1+|P|)\left|D_{P} A_{P_{j}}^{i}(P)\right| \leq C .
$$

Finally, we state a linear problem corresponding to (5.13)-(5.16). Namely, we use (5.20) to find that, for $i=1, \ldots, n$,

$$
A^{i}(D u(x))=\sum_{j=1}^{n} \tilde{a}_{i j}(x) u_{x_{j}}(x), \quad \tilde{a}_{i j}(x)=\int_{0}^{1} A_{p_{j}}^{i}(t D u(x)) d t .
$$

We replace $u \equiv \varphi-q^{+} x_{n}$ in the definition of the coefficients $\tilde{a}_{i j}$ by $\psi-q^{+} x_{n}$ for $\psi \in \mathcal{K}_{M}$ to define

$$
a_{i j}(x)=a_{i j}^{(\psi)}(x)=\int_{0}^{1} A_{p_{j}}^{i}\left(t\left(D \psi(x)-q^{+} e_{n}\right)\right) d t \quad \text { for } x \in \Omega, \quad i, j=1, \ldots, n .
$$

The ellipticity (5.19) of $A(P)$ implies that the coefficients $\left\{a_{i j}^{(\psi)}(x)\right\}$ satisfy the ellipticity condition (3.35) for any $x \in \Omega$.

Also, from (5.17) and (5.21),

$$
\begin{aligned}
& a_{i j}^{(\psi)}(x) \\
& =\int_{0}^{1}\left\{\tilde{\rho}\left(\left|t D \psi(x)+(1-t) q^{+} e_{n}\right|^{2}\right) \delta_{i}^{j}\right. \\
& \left.\quad+2 \tilde{\rho}^{\prime}\left(\left|t D \psi(x)+(1-t) q^{+} e_{n}\right|^{2}\right)\left(t \psi_{x_{i}}(x)+(1-t) q^{+} \delta_{i}^{n}\right)\left(t \psi_{x_{j}}(x)+(1-t) q^{+} \delta_{j}^{n}\right)\right\} d t
\end{aligned}
$$

for $x \in \Omega$, where $\delta_{i}^{j}=1$ if $i=j$ and $\delta_{i}^{j}=0$ if $i \neq j$. In particular, we have $a_{i j}=a_{j i}$. 
We note that, for $\breve{\psi}_{0}=q^{+} x_{n}$, the corresponding coefficients $\breve{a}_{i j}$ defined by (5.22) are constants and satisfy

$$
\breve{a}_{i j}=\kappa_{i} \delta_{i}^{j} \quad \text { for } i, j=1, \ldots, n,
$$

where $\kappa_{i}=\tilde{\rho}\left(\left(q^{+}\right)^{2}\right)$ for $i=1, \ldots, n-1$; and $\kappa_{n}=\phi^{\prime}\left(q^{+}\right)$for $\phi(s)=\tilde{\rho}\left(s^{2}\right) s$. We have

$$
\lambda \leq \kappa_{i} \leq \lambda^{-1} \quad \text { for } i=1, \ldots, n .
$$

Now, for $\psi \in \mathcal{K}_{M}$, we use (5.22) and (4.1)-(4.3) to obtain

$$
\left\|a_{i j}^{(\psi)}-\breve{a}_{i j}\right\|_{0, \alpha, \Omega} \leq C M \sigma \text {. }
$$

Thus we formulate the following conormal fixed boundary elliptic problem:

$$
\begin{aligned}
& \sum_{i, j=1}^{n}\left(a_{i j}^{(\psi)} u_{x_{j}}\right)_{x_{i}}=0 \quad \text { in } \Omega^{+}(\psi), \\
& \sum_{i, j=1}^{n} a_{i j}^{(\psi)} u_{x_{j}} \nu_{i}=g_{\psi}(x) \quad \text { on } \quad S_{\psi}, \\
& \sum_{i, j=1}^{n} a_{i j}^{(\psi)} u_{x_{j}} \nu_{i}=-\tilde{\rho}\left(\left(q^{+}\right)^{2}\right) q^{+} \nu \cdot e_{n} \quad \text { on } \partial_{l} \Omega^{+}(\psi), \\
& \lim _{R \rightarrow \infty}\|u\|_{C\left(\Omega^{+}(\psi) \cap\left\{x_{n}>R\right\}\right)}=0 .
\end{aligned}
$$

Since the coefficients are only $C^{\alpha}$, we can expect to find only a weak solution $u \in C^{1, \alpha}\left(\overline{\Omega^{+}(\psi)}\right)$ of (5.25)-(5.28) in the sense that $u(x)$ satisfies (5.28) and, for any $w \in C_{c}^{1}\left(\mathbf{R}^{n}\right)$,

$$
\begin{aligned}
\int_{\Omega^{+}(\psi)} \sum_{i, j=1}^{n} a_{i j}^{(\psi)} & u_{x_{j}} w_{x_{i}} d x+\int_{S_{\psi}} g_{\psi} w d \mathcal{H}^{n-1} \\
& -\int_{\partial_{l} \Omega^{+}(\psi)} \tilde{\rho}\left(\left(q^{+}\right)^{2}\right) q^{+} \nu \cdot e_{n} w d \mathcal{H}^{n-1}=0 .
\end{aligned}
$$

We will determine the iteration map $J(\psi)=\varphi$ by solving (5.25)-(5.28) for $u$, extending $u$ from $\Omega^{+}(\psi)$ to $\Omega$ so that $u+q^{+} x_{n} \in \mathcal{K}_{M}$, and defining $\varphi=u+q^{+} x_{n}$. A fixed point of this map is obviously a solution of Problem (TFB) in $\Omega$. In Sections $6-7$, we prove the existence of such a map $J$, as well as its fixed point.

\section{Fixed Boundary Problems in a bounded domain $\Omega_{R}^{+}(\psi)$}

In order to find a solution of (5.25)-(5.28) in the unbounded domain $\Omega^{+}(\psi)$, we first solve the corresponding problem in the bounded domain

$$
\Omega_{R}^{+}(\psi):=\Omega^{+}(\psi) \cap\left\{x_{n}<R\right\}, \quad R>L,
$$

and then pass to the limit as $R \rightarrow \infty$. Thus we consider the following problem:

$$
\begin{aligned}
& \sum_{i, j=1}^{n}\left(a_{i j}^{(\psi)} u_{x_{j}}\right)_{x_{i}}=0 \quad \text { in } \Omega_{R}^{+}(\psi), \\
& \sum_{i, j=1}^{n} a_{i j}^{(\psi)} u_{x_{j}} \nu_{i}=g_{\psi}(x) \quad \text { on } \quad S_{\psi}, \\
& \sum_{i, j=1}^{n} a_{i j}^{(\psi)} u_{x_{j}} \nu_{i}=-\tilde{\rho}\left(\left(q^{+}\right)^{2}\right) q^{+} \nu \cdot e_{n} \quad \text { on } \quad \partial_{l} \Omega_{R}^{+}(\psi), \\
& u=0 \quad \text { on } \quad \partial \Omega_{R}^{+}(\psi) \cap\left\{x_{n}=R\right\},
\end{aligned}
$$

where $a_{i j}^{(\psi)}$ are defined by (5.21) and $\partial_{l} \Omega_{R}^{+}(\psi)=\partial_{l} \Omega^{+} \cap \partial \Omega_{R}^{+}(\psi)$. 
We first note several properties of $g_{\psi}$ which will be used later.

Lemma 6.1. There exists $C>0$ depending only on the data and independent of $M$ and $R$ such that

$$
\left\|g_{\psi}\right\|_{0, \alpha, \Omega_{1}} \leq C \sigma
$$

This is proved in [5, (4.48), page 334]. Another property of $g_{\psi}$ is

\section{Lemma 6.2.}

$$
\int_{S_{f}} g_{\psi} d \mathcal{H}^{n-1}=\tilde{\rho}\left(\left(q^{+}\right)^{2}\right) q^{+} \int_{\partial_{l} \Omega_{R}^{+}} \nu \cdot e_{n} d \mathcal{H}^{n-1}
$$

Proof. Note that

$$
0=\int_{\Omega_{R}} \Delta\left(x_{n}\right)=-\int_{\Lambda \times\left\{x_{n}=R\right\}} e_{n} \cdot \nu d x^{\prime}-\int_{S_{f}} e_{n} \cdot \nu d \mathcal{H}^{n-1}-\int_{\partial_{l} \Omega_{R}^{+}} e_{n} \cdot \nu d \mathcal{H}^{n-1},
$$

where $\nu$ is the inward unit normal. Since $\nu=-e_{n}$ on $\Lambda \times\left\{x_{n}=R\right\}$, then, from the last equality, we get

We also note that

$$
\int_{S_{f}} e_{n} \cdot \nu d \mathcal{H}^{n-1}+\int_{\partial_{l} \Omega_{R}^{+}} e_{n} \cdot \nu d \mathcal{H}^{n-1}=|\Lambda|
$$

$$
\int_{S_{f}} G_{\psi} d \mathcal{H}^{n-1}=\int_{\partial_{o} \Omega} \rho\left(\left|D \varphi^{-}\right|^{2}\right) D \varphi^{-} \cdot \nu d \mathcal{H}^{n-1}
$$

since $G_{\psi}$ is defined by $(5.8)$ and $\varphi^{-} \in C^{1, \alpha}\left(\overline{\Omega \backslash \Omega_{R}^{+}(\psi)}\right)$ is a weak solution of $(1.1)$ in $\Omega \backslash \overline{\Omega_{R}^{+}(\psi)}$ and satisfies the boundary condition (2.19).

Then we have

$$
\begin{aligned}
& \int_{S_{f}} g_{\psi} d \mathcal{H}^{n-1}-\tilde{\rho}\left(\left(q^{+}\right)^{2}\right) q^{+} \int_{\partial_{l} \Omega_{R}^{+}} \nu \cdot e_{n} d \mathcal{H}^{n-1} \\
& =\int_{S_{f}} G_{\psi} d \mathcal{H}^{n-1}-\tilde{\rho}\left(\left(q^{+}\right)^{2}\right) q^{+}\left(\int_{S_{f}} \nu \cdot e_{n} d \mathcal{H}^{n-1}+\int_{\partial_{l} \Omega_{R}^{+}} \nu \cdot e_{n} d \mathcal{H}^{n-1}\right) \\
& =\int_{S_{f}} G_{\psi} d \mathcal{H}^{n-1}-\tilde{\rho}\left(\left(q^{+}\right)^{2}\right) q^{+}|\Lambda|=0
\end{aligned}
$$

where we used (6.7) and the fact that $q^{+}$satisfies (2.26) and (2.27).

6.1. Existence of Solutions and Their Uniform Estimates Independent of $R$ for the Linear Fixed Boundary Problem in $\Omega_{R}^{+}(\psi)$. In this section, we fix $\psi \in \mathcal{K}_{M}$ and write $\Omega_{R}^{+}$ for $\Omega_{R}^{+}(\psi)$ and $a_{i j}$ for $a_{i j}^{(\psi)}$ to simplify the presentation. Also, $C$ denotes a universal constant depending only on the data and independent of $M$ and $R$, which may be different at each occurrence. Furthermore, there exists $\sigma_{0}>0$ depending only on $M$ such that, when $\sigma \leq \sigma_{0}$, $S_{\psi} \subset\left\{-1 / 10<x_{n}<1 / 10\right\}$ by (5.5); we always assume $\sigma \leq \sigma_{0}$ below.

Lemma 6.3. Let $\sigma \in\left(0, \sigma_{0}\right)$ be sufficiently small, depending only on the data and $M$. Let $u \in H^{1}\left(\Omega_{R}^{+}\right), R>L$, be a weak solution of (6.1)-(6.4) in the sense that

(i) $u=0$ on $\partial \Omega_{R}^{+} \cap\left\{x_{n}=R\right\}$ as the trace;

(ii) for any $w \in H^{1}\left(\Omega_{R}^{+}\right)$satisfying $w=0$ on $\partial \Omega_{R}^{+} \cap\left\{x_{n}=R\right\}$ in the sense of traces,

$$
\int_{\Omega_{R}^{+}} \sum_{i, j=1}^{n} a_{i j} u_{x_{j}} w_{x_{i}} d x+\int_{S_{f}} g_{\psi} w d \mathcal{H}^{n-1}-\int_{\partial_{l} \Omega_{R}^{+}} \tilde{\rho}\left(\left(q^{+}\right)^{2}\right) q^{+} \nu \cdot e_{n} w d \mathcal{H}^{n-1}=0
$$

Then

$$
\|D u\|_{L^{2}\left(\Omega_{R}^{+}\right)} \leq C \sigma .
$$


Proof. We use the approach in the proof of [5, Lemma 4.2]. We first choose $w=u$ in (6.8) to obtain

$$
\int_{\Omega_{R}^{+}} \sum_{i, j=1}^{n} a_{i j} u_{x_{j}} u_{x_{i}} d x=-\int_{S_{f}} g_{\psi} u d \mathcal{H}^{n-1}+\int_{\partial_{l} \Omega_{R}^{+}} \tilde{\rho}\left(\left(q^{+}\right)^{2}\right) q^{+} \nu \cdot e_{n} u d \mathcal{H}^{n-1} .
$$

Now, if $Q$ is a constant which will be chosen below, we use Lemma 6.2 to obtain

$$
\int_{\Omega_{R}^{+}} \sum_{i, j=1}^{n} a_{i j} u_{x_{j}} u_{x_{i}} d x=-\int_{S_{f}} g_{\psi}(u-Q) d \mathcal{H}^{n-1}-\int_{\partial_{l} \Omega_{L}^{+}} \tilde{\rho}\left(\left(q^{+}\right)^{2}\right) q^{+} \nu \cdot e_{n}(u-Q) d \mathcal{H}^{n-1},
$$

where we used that $\nu \cdot e_{n}=0$ on $\partial_{l} \Omega_{R}^{+} \backslash \partial_{l} \Omega_{L}^{+}$by (2.14) and (2.17).

Choosing $Q=(u)_{L}:=\frac{1}{\left|\Omega_{L}^{+}(\psi)\right|} \int_{\Omega_{L}^{+}(\psi)} u(x) d x$ and using the $L^{2}$ estimate of the boundary traces of functions in the Sobolev space $H^{1}\left(\Omega_{L}^{+}(\psi)\right),(6.5)$, and $\left|\nu \cdot e_{n}\right| \leq C \sigma$ by (2.16), we obtain

$$
\begin{aligned}
\int_{\Omega_{R}^{+}} \sum_{i, j=1}^{n} a_{i j} u_{x_{j}} u_{x_{i}} d x \leq & \left(\int_{S_{f}} g_{\psi}^{2} d \mathcal{H}^{n-1}\right)^{1 / 2}\left(\int_{S_{f}}\left(u-(u)_{L}\right)^{2} d \mathcal{H}^{n-1}\right)^{1 / 2} \\
& +\tilde{\rho}\left(\left(q^{+}\right)^{2}\right) q^{+}\left(\int_{\partial_{l} \Omega_{L}^{+}}\left|\nu \cdot e_{n}\right|^{2} d \mathcal{H}^{n-1}\right)^{1 / 2}\left(\int_{\partial_{l} \Omega_{L}^{+}}\left(u-(u)_{L}\right)^{2} d \mathcal{H}^{n-1}\right)^{1 / 2} \\
\leq & C \sigma\left(\int_{\Omega_{L}^{+}(\psi)}\left(\left(u-(u)_{L}\right)^{2}+|D u|^{2}\right) d x\right)^{1 / 2} \\
\leq & C \sigma\left(\int_{\Omega_{L}^{+}(\psi)}|D u|^{2} d x\right)^{1 / 2}
\end{aligned}
$$

where, in the last estimate, we used the Poincaré inequality in the domain $\Omega_{L}^{+}(\psi)$, and thus the constant $C$ depends only on $n, \mathrm{~L}$, the norms in (2.15) and (2.16), and $\left\|f_{\psi}\right\|_{1, \alpha, \Lambda}$. On the other hand, if $\sigma<\frac{1}{M}$, then $\left\|f_{\psi}\right\|_{1, \alpha, \Lambda} \leq C$ with $C>0$ independent of $M, R$, and $\psi \in \mathcal{K}_{M}$. Now, using (3.35), we get

$$
\int_{\Omega_{R}^{+}}|D u|^{2} d x \leq \frac{1}{\lambda} \int_{\Omega_{R}^{+}} \sum_{i, j=1}^{n} a_{i j} u_{x_{j}} u_{x_{i}} d x \leq \frac{C}{\lambda} \sigma\left(\int_{\Omega_{R}^{+}}|D u|^{2} d x\right)^{1 / 2} .
$$

This completes the proof.

Since $\partial_{l} \Omega \cap \partial \Omega_{R}^{+}$is $C^{1, \alpha}$, then, by [36], the weak solution $u$ of (6.1)-(6.4) is in $C^{1, \alpha}\left(\bar{\Omega}_{R}^{+} \backslash\left(\overline{S_{\psi}} \cup\left\{x_{n}=R\right\}\right)\right)$.

Lemma 6.4. Let $\sigma$ and $u$ be as in Lemma 6.3. Then, for any $x_{n} \in(L, R)$, there exists $x^{\prime} \in \bar{\Lambda}$ such that $u\left(x^{\prime}, x_{n}\right)=0$.

Proof. We follow the scheme of the proof [5, Lemma 4.3, Step 2]. Fix $z \in(L, R)$. Suppose that there is no $x^{\prime} \in \bar{\Lambda}$ such that $u\left(x^{\prime}, z\right)=0$. Then we can assume $u\left(x^{\prime}, z\right)>0$ for all $x^{\prime} \in \bar{\Lambda}$, since the case $u\left(x^{\prime}, z\right)<0$ for all $x^{\prime} \in \bar{\Lambda}$ can be handled similarly. From the compactness of $\Lambda$ and continuity of $u$, there exists a constant $\kappa>0$ such that

$$
u\left(x^{\prime}, z\right) \geq \kappa>0 \quad \text { for all } \quad x^{\prime} \in \bar{\Lambda} .
$$


Consider domain $\mathcal{D}:=\Lambda \times(z, R)$. Then $u \in C(\overline{\mathcal{D}}) \cap C^{2}(\overline{\mathcal{D}} \backslash W)$, with $W=\partial_{l} \Omega \cap\left\{x_{n}=R\right\}$, and $u$ is a weak solution of

$$
\begin{array}{ll}
\sum_{i, j=1}^{n}\left(a_{i j} u_{x_{j}}\right)_{x_{i}}=0 & \text { in } \quad \mathcal{D}, \\
\sum_{i, j=1}^{n} a_{i j} u_{x_{j}} \nu_{i}=0 & \text { on } \quad \partial_{l} \mathcal{D}=\partial \mathcal{D} \cap \partial_{l} \Omega \cap\left\{x_{n}<R\right\}, \\
u \geq \kappa>0 & \text { on } \quad \partial \mathcal{D} \cap\left\{x_{n}=z\right\}, \\
u=0 & \text { on } \quad \partial \mathcal{D} \cap\left\{x_{n}=R\right\} .
\end{array}
$$

Thus, by the maximum principle, $u \geq 0$ in $\mathcal{D}$. In particular,

$$
u_{\nu}=-u_{x_{n}} \geq 0 \quad \text { on }\left(\partial \mathcal{D} \cap\left\{x_{n}=R\right\}\right) \backslash W .
$$

By the strong maximum principle [21, Theorem 8.19], $u>0$ in the interior of $\mathcal{D}$. Also, denoting $\mathcal{D}_{\delta}=\left\{x \in \mathcal{D}: \operatorname{dist}\left(x, \partial_{l} \Omega\right)>\delta\right\}$, where $\delta>0$ is fixed to be small so that $\mathcal{D}_{\delta} \neq \emptyset$, we have

$$
a_{i j} \in C^{0, \alpha}\left(\overline{\mathcal{D}_{\delta / 2}}\right), \quad u \in C^{1, \alpha}\left(\overline{\mathcal{D}_{\delta / 2}}\right) .
$$

Thus, by [5, Lemma A.1],

$$
u_{\nu}=-u_{x_{n}}>0 \quad \text { on } \overline{\mathcal{D}}_{\delta} \cap\left\{x_{n}=R\right\} .
$$

Since $u=0$ on $\Lambda \times\left\{x_{n}=R\right\}$ and $a_{n n} \geq \lambda>0$ from the ellipticity, we conclude from (6.10) and (6.11) that

$$
\sum_{i, j=1}^{n} a_{i j} u_{x_{j}} \nu_{i}=-a_{n n} u_{x_{n}} \begin{cases}\geq 0 & \text { on } \quad\left(\partial \mathcal{D} \cap\left\{x_{n}=R\right\}\right) \backslash W, \\ >0 & \text { on } \quad \overline{\mathcal{D}}_{\delta} \cap\left\{x_{n}=R\right\} .\end{cases}
$$

Since $a_{n n}$ and $u_{x_{n}}$ are continuous on $\overline{\mathcal{D}}_{\delta} \cap\left\{x_{n}=R\right\}$, we conclude

$$
\int_{\bar{\Omega} \cap\left\{x_{n}=R\right\}} \sum_{i, j=1}^{n} a_{i j} u_{x_{j}} \nu_{i} d \mathcal{H}^{n-1}>0 .
$$

On the other hand, from (6.1)-(6.3),

$$
\begin{aligned}
0 & =\int_{\Omega_{R}^{+}} \sum_{i, j=1}^{n}\left(a_{i j}^{\psi} u_{x_{j}}\right)_{x_{i}} d x \\
& =\left(\int_{S_{\psi}}+\int_{\partial_{l} \Omega_{R}^{+}}+\int_{\bar{\Omega} \cap\left\{x_{n}=R\right\}}\right) \sum_{i, j=1}^{n} a_{i j} u_{x_{j}} \nu_{i} d \mathcal{H}^{n-1} \\
& =\int_{S_{\psi}} g_{\psi} d \mathcal{H}^{n-1}-\int_{\partial_{l} \Omega_{R}^{+}} \tilde{\rho}\left(\left(q^{+}\right)^{2}\right) q^{+} \nu \cdot e_{n} d \mathcal{H}^{n-1}+\int_{\bar{\Omega} \cap\left\{x_{n}=R\right\}} \sum_{i, j=1}^{n} a_{i j} u_{x_{j}} \nu_{i} d \mathcal{H}^{n-1} \\
& =\int_{\bar{\Omega} \cap\left\{x_{n}=R\right\}} \sum_{i, j=1}^{n} a_{i j} u_{x_{j}} \nu_{i} d \mathcal{H}^{n-1},
\end{aligned}
$$

where the last equality follows from Lemma 6.2. Thus we conclude a contradiction with (6.12), which completes the proof.

From (2.14)-(2.17), there exist $r>0$ and $C>0$ with the following properties: For any $x_{0} \in \partial_{l} \Omega \cap\left\{x_{n}>-1 / 2\right\}$, there exists an orthonormal coordinate system $\left(y_{1}, \ldots, y_{n}\right)$ with $y_{n}=x_{n}$ and a function $\zeta: \mathbf{R}^{n-1} \rightarrow \mathbf{R}$ such that

$$
\Omega \cap B_{10 r}\left(x_{0}\right)=\left\{y_{1}>\zeta\left(y_{2}, \ldots, y_{n}\right)\right\} \cap B_{10 r}\left(x_{0}\right), \quad\|\zeta\|_{1, \alpha, \mathbf{R}^{n-1}} \leq C, \quad D \zeta\left(x_{0}\right)=0 .
$$


Proposition 6.5. Let $u(x)$ be as in Lemma 6.3. If $\sigma$ is sufficiently small, depending only on the data and $M$, then, for any $x^{0}=\left(x_{1}^{0}, \ldots, x_{n}^{0}\right) \in \overline{\Omega_{R-10 r}^{+}}$with $R-10 r>x_{n}^{0} \geq L+1$,

$$
\|D u\|_{0, \alpha, B_{r}\left(x_{0}\right) \cap \Omega_{R}^{+}} \leq C\|D u\|_{L^{2}\left(B_{4 r}\left(x_{0}\right) \cap \Omega_{R}^{+}\right)} .
$$

Proof. We use the fact that $u-K$ also satisfies equation (6.1) and the conormal boundary conditions (6.2) and (6.3) for any constant $K \in \mathbf{R}$.

When $x_{0}$ satisfies $B_{2 r}\left(x_{0}\right) \subset \Omega_{R}^{+}$, we apply [26, Theorem 3.13] to $u-K$ and get

$$
\|D u\|_{0, \alpha, B_{r}\left(x_{0}\right) \cap \Omega_{R}^{+}} \leq C\|u-K\|_{L^{2}\left(B_{4 r}\left(x_{0}\right) \cap \Omega_{R}^{+}\right)} .
$$

When $x_{0} \in \partial_{l} \Omega^{+}$with $R-10 r>x_{n}^{0} \geq L+1$, then, since the right-hand side of (6.3) vanishes on $\partial_{l} \Omega^{+} \cap\left\{x_{n}>L\right\}$, we use (5.24) and (a linear version of) the estimates for the conormal derivative problem [36], applied to $u-K$, to obtain

$$
\|D u\|_{0, \alpha, B_{r}\left(x_{0}\right) \cap \Omega_{R}^{+}} \leq C\|u-K\|_{C^{0}\left(B_{2 r}\left(x_{0}\right) \cap \Omega_{R}^{+}\right)} .
$$

Now we use the standard estimates [21, Theorem 8.17] for the equations of divergence form (extended to the case of local estimates near the boundary for the conormal problem, see e.g. [38, Chapter 4, Section 10] where these modifications are indicated in the parabolic case; note that the Lipschitz regularity of the boundary in our case allows to use the weak form of the conormal problem) to obtain

$$
\|u-K\|_{C^{0}\left(B_{2 r}\left(x_{0}\right) \cap \Omega_{R}^{+}\right)} \leq C\|u-K\|_{L^{2}\left(B_{4 r}\left(x_{0}\right) \cap \Omega_{R}^{+}\right)} .
$$

That is, (6.15) is satisfied for the case $x_{0} \in \partial_{l} \Omega_{R}^{+}$.

Now choosing $K=\frac{1}{\left|B_{4 r}\left(x_{0}\right) \cap \Omega_{R}^{+}\right|} \int_{B_{4 r}\left(x_{0}\right) \cap \Omega_{R}^{+}} u d x$, using the Poincare inequality, and noting that the constant in the Poincare inequality can be chosen independent of $x_{0} \in \Omega_{R}^{+}$by (6.13), we complete the proof.

Proposition 6.6. Let $\sigma$ and $u(x)$ be as in Lemma 6.3. Then

$$
\|u\|_{C^{0}\left(\overline{\Omega_{R}^{+}}\right)} \leq C \sigma .
$$

Proof. Lemma 6.3 and Proposition 6.5 imply that

$$
|D u| \leq C \sigma \quad \text { on } \Omega_{R}^{+} \cap\left\{L+1<x_{n}<R-10 r\right\} .
$$

Combining this with Lemma 6.4, we have

$$
|u| \leq C \sigma \quad \text { on } \Omega_{R}^{+} \cap\left\{x_{n} \in(L+1, R-10 r)\right\} .
$$

To extend this bound to the domains $\Omega_{R}^{+} \cap\left\{R-10 r<x_{n}<R\right\}=\Lambda \times(R-10 r, R)$ and $\Omega_{L}^{+}$, we note that these domains are of the fixed size and structure (cylinder and a small perturbation cylinder of the form $\Lambda \times(a, b)$, respectively). Note that, on the boundary parts $\left\{x_{n}=L\right\} \subset \partial \Omega_{L}^{+}$ and $\left\{x_{n}=R-10 r\right\} \subset \partial(\Lambda \times(R-10 r, R))$, we have $|u| \leq C \sigma$, as we proved above. Moreover, the right-hand sides of (6.2) and (6.3) are estimated in $L^{\infty}$ by $C \sigma$ from (2.16) and (6.5), respectively. Thus we can use the standard estimates [21, Theorem 8.15] for the equations of divergence form (extended to the case when we have the conormal boundary conditions on a part of the boundary, see e.g. [38, Chapter 4, Section 10]).

Proposition 6.7. If $\sigma$ is sufficiently small, there exists a unique weak solution $u \in H^{1}\left(\Omega_{R}^{+}\right)$of (6.1)-(6.4). Furthermore, $u \in C^{1, \alpha}\left(\overline{\Omega_{R-10 r}^{+}}\right)$satisfies (6.9) and

$$
\|u\|_{1, \alpha, \Omega_{R-10 r}^{+}} \leq C \sigma
$$


Proof. Since the map $\Psi$ in (2.14) satisfies (2.16), then, if $\sigma$ is sufficiently small, there exists the inverse map $\Phi: \mathbf{R}^{n} \rightarrow \mathbf{R}^{n}$ of $\Psi$. The map $\Phi$ satisfies

$$
\|\Phi-I d\|_{2, \alpha, \mathbf{R}^{n}} \leq C \sigma .
$$

Then, from (2.14),

$$
\Phi\left(\partial_{l} \Omega\right) \subset \partial \Lambda \times(-\infty, \infty) .
$$

Note that, if $\sigma$ is small, then, by (2.16), (4.5), and (5.4), we conclude that the set $\Phi\left(\Omega^{+}(\psi)\right)=$ $\left\{\psi \circ \Psi<\varphi^{-} \circ \Psi\right\} \cap(\Lambda \times \mathbf{R})$ has the form

$$
\Phi\left(\Omega^{+}(\psi)\right)=\left\{x_{n}>\tilde{f}\left(x^{\prime}\right)\right\} \cap(\Lambda \times \mathbf{R}) \quad \text { with } \quad\|\tilde{f}\|_{1, \alpha, \mathbf{R}^{n-1}} \leq C M \sigma .
$$

Now we consider a map

$$
\tilde{\Phi}=\Phi^{1} \circ \Phi: \mathbf{R}^{n} \rightarrow \mathbf{R}^{n}, \quad \text { for } \Phi^{1}\left(x^{\prime}, x_{n}\right)=\left(x^{\prime}, x_{n}-\eta\left(x_{n}\right) \tilde{f}\left(x^{\prime}\right)\right),
$$

where $\eta \in C^{\infty}(\mathbf{R})$ is nonincreasing and satisfies $\eta \equiv 1$ on $(-\infty, 1 / 8)$ and $\eta \equiv 0$ on $(1 / 4, \infty)$. From this definition, we have

$$
\|\tilde{\Phi}-I d\|_{1, \alpha, \mathbf{R}^{n}} \leq C M \sigma .
$$

Thus, for small $M \sigma$, the map $\tilde{\Phi}$ is invertible and

$$
\left\|\tilde{\Phi}^{-1}-I d\right\|_{1, \alpha, \mathbf{R}^{n}} \leq C M \sigma .
$$

In particular, for small $M \sigma$

$$
\|\tilde{\Phi}-I d\|_{1, \alpha, \mathbf{R}^{n}} \leq \frac{1}{10}, \quad\left\|\tilde{\Phi}^{-1}-I d\right\|_{1, \alpha, \mathbf{R}^{n}} \leq \frac{1}{10} .
$$

Moreover, if $M \sigma$ is small, then

$$
\Lambda \times(0, \rho)=\tilde{\Phi}\left(\Omega_{R}^{+} \cap\left\{x_{n}<\rho\right\}\right) \quad \text { for } \rho>1 / 4 .
$$

Define the function $v(y):=u(x)=u\left(\tilde{\Phi}^{-1}(y)\right)$ for $y \in \mathcal{C}_{R}:=\Lambda \times(0, R)$. Then $u$ is a weak solution of the conormal problem (6.1)-(6.4) if and only if $v \in H^{1}\left(\mathcal{C}_{R}\right)$ is a weak solution of the corresponding problem of the form (3.1)-(3.3) in $\mathcal{C}_{R}$. To obtain the expressions of the coefficients $a_{i j}$ and the right-hand sides $f, g_{1}$, and $g_{2}$ of problem (3.1)-(3.2) for $v$, in terms of the data of problem (6.1)-(6.3) and the map $\tilde{\Phi}$, we make the change of variables $y=\tilde{\Phi}(x)$ in (6.8). Then we get

$$
\left\|a_{i j}-\kappa_{i} \delta_{i}^{j}\right\|_{0, \alpha, \mathcal{C}_{R}} \leq C M \sigma \quad \text { for } i, j=1, \ldots, n,
$$

with $\kappa_{i}$ defined by (5.23), where we used (5.24) and (6.22). In this case, the right-hand side in (3.1) is $f=0$; to estimate $g_{1}$ and $g_{2}$ in (3.2), we use (2.16), (6.5), (6.22) to obtain

$$
\left\|g_{k}\right\|_{0, \alpha, \Sigma_{k}} \leq C \sigma, \quad k=1,2 .
$$

Also, from (6.4) and (6.23), we see that $h=0$ in (3.3) for $v$.

Now the existence and uniqueness of $v(y)$ follows from Theorem 3.1. Thus $u(x):=v(\tilde{\Phi}(x))$ is a unique weak solution of (6.1)-(6.4). Estimate (6.9) follows from Lemma 6.3.

It remains to prove (6.17). For any $x \in \overline{\Omega_{R}^{+}} \cap\left\{1 \leq x_{n} \leq R-10 r\right\}$, the standard interior estimates [21, Theorem 8.32] and the estimates for oblique derivative problems [36] imply

$$
\|u\|_{1, \alpha, B_{r}\left(x_{0}\right) \cap \Omega_{R}^{+}} \leq C\left(\|u\|_{C^{0}\left(B_{4 r}\left(x_{0}\right) \cap \Omega_{R}^{+}\right)}+\left\|\tilde{\rho}\left(\left(q^{+}\right)^{2}\right) q^{+} \nu \cdot e_{n}\right\|_{0, \alpha, B_{r}\left(x_{0}\right) \cap \partial \Omega_{R}^{+}} \leq C \sigma,\right.
$$

where we used Proposition 6.6 and (2.16). Thus it suffices to obtain an estimate in $\Omega_{R}^{+} \cap\left\{x_{n} \leq 1\right\}$. By (6.22) and (6.23), it is sufficient to show that

$$
\|v\|_{1, \alpha, \Lambda \times(0,1)} \leq C \sigma
$$

since the similar estimate then holds for $\|u\|_{1, \alpha, \Omega_{R}^{+} \cap\left\{x_{n} \leq 1\right\}}$.

To show (6.27), we note that using (6.22), (6.23), and Proposition 6.6 yields

$$
\|v\|_{L^{2}(\Lambda \times(0,2))} \leq 2\|u\|_{L^{2}\left(\Omega_{R}^{+} \cap\left\{x_{n}<2\right\}\right)} \leq C \sigma .
$$


Now estimate (3.8), applied to $v$ in the cylinder $\Lambda \times(0,2)$, with the use of (6.25), implies (6.27), if $\sigma$ is small depending only on $M$ and the data.

\section{Existence of Solutions of the Free Boundary Problem}

We now show the existence of a solution of the free boundary problem.

7.1. Solutions of the Fixed Boundary Problem in the Infinite Nozzle. We first have

Proposition 7.1. If $\sigma$ is sufficiently small, then there exists a unique weak solution $u \in$ $C^{1, \alpha}\left(\overline{\Omega^{+}(\psi)}\right)$ of (5.25)-(5.28). This solution satisfies

$$
\begin{aligned}
& \|D u\|_{L^{2}\left(\Omega^{+}(\psi)\right)} \leq C \sigma, \\
& \|u\|_{1, \alpha, \Omega^{+}(\psi)} \leq C \sigma, \\
& \lim _{\rho \rightarrow \infty}\|u\|_{1, \alpha, \Omega \cap\left\{x_{n}>\rho\right\}}=0 .
\end{aligned}
$$

Moreover, we have

(i) $u=\lim _{R \rightarrow \infty} u_{R}$, where $u_{R}$ is the unique solution of (6.1)-(6.4) for each $R>0$ and the convergence is in $C^{1, \beta}$ on any compact subset of $\overline{\Omega^{+}(\psi)}$ for any $0 \leq \beta<\alpha$;

(ii) For every $R \geq L$, there exists $x^{\prime} \in \bar{\Lambda}$ such that $u\left(x^{\prime}, R\right)=0$.

Proof. We first show that any weak solution $u \in C^{1, \alpha}\left(\overline{\Omega^{+}(\psi)}\right)$ of $(5.25)-(5.28)$ satisfies (7.3). From (5.28), for any $\varepsilon>0$, there exists $\rho>L$ such that

$$
\|u\|_{C\left(\Omega \cap\left\{x_{n}>\rho\right\}\right)} \leq \varepsilon .
$$

Note that (2.17) implies that the right-hand side of (5.27) vanishes on $\partial_{l} \Omega^{+}(\psi) \cap\left\{x_{n}>\rho\right\}$. Then the standard estimates [21, Theorem 6.2, Lemma 6.29] imply that, for $x=\left(x^{\prime}, x_{n}\right)$ with $x_{n}>L+2 r$,

Thus, (7.3) follows.

$$
\|u\|_{1, \alpha, B_{r}(x) \cap \Omega} \leq C\|u\|_{0,0, B_{2 r}(x) \cap \Omega} \leq \varepsilon .
$$

Now we prove the uniqueness of weak solutions of $(5.25)-(5.28)$ in $C^{1, \alpha}\left(\overline{\Omega^{+}(\psi)}\right)$. Let $u, v \in$ $C^{1, \alpha}\left(\overline{\Omega^{+}(\psi)}\right)$ be two solutions of (5.25)-(5.28). Then, for any $R>L+\operatorname{diam}(\Lambda)$, define

$$
\zeta(x):=\eta_{R}(x)(u(x)-v(x)),
$$

where $\eta_{R}(x)=\eta(|x| / R)$ and $\eta \in C^{\infty}\left(\overline{\mathbf{R}_{+}}\right)$is a nonnegative function such that $\eta \equiv 1$ on $[0,1]$. Then $\zeta \in C_{c}^{1}\left(\mathbf{R}^{n} \cap \overline{\Omega^{+}}\right)$. Using $\zeta$ as a test function in (5.29) for $u$ and $v$ respectively and subtracting them, we get

$$
0=\int_{\Omega^{+}} \sum_{i, j=1}^{n} a_{i j}^{(\psi)}\left(\left(u_{x_{i}}-v_{x_{i}}\right)\left(u_{x_{j}}-v_{x_{j}}\right) \eta_{R}+(u-v)\left(u_{x_{j}}-v_{x_{j}}\right) \partial_{x_{i}} \eta_{R}\right) d x .
$$

Thus,

$$
\int_{\Omega^{+}} \sum_{i, j=1}^{n} a_{i j}^{(\psi)}\left(u_{x_{i}}-v_{x_{i}}\right)\left(u_{x_{j}}-v_{x_{j}}\right) \eta_{R} d x \leq \frac{C}{R} \int_{\Omega^{+} \cap\left(B_{2 R}(0) \backslash B_{R}(0)\right)}(|D u|+|D v|)(|u|+|v|) d x .
$$

Note that $\left|\Omega^{+} \cap\left(B_{2 R}(0) \backslash B_{R}(0)\right)\right| \leq 2 R|\Lambda|$. Thus, using the ellipticity of $a_{i j}^{(\psi)}$, we get

$$
\lambda \int_{\Omega^{+} \cap B_{R}(0)}|D u-D v|^{2} d x \leq C\left(\|u\|_{1,0, \Omega \backslash B_{R}(0)}+\|v\|_{1,0, \Omega \backslash B_{R}(0)}\right)
$$

for large $R$. Now we send $R \rightarrow \infty$ and use (7.3) to get

$$
\lambda \int_{\Omega_{R}^{+}}|D u-D v|^{2} d x=0 .
$$

Thus, $u-v=$ const., which yields $u=v$ by using (7.3). 
It remains to prove the existence of a weak solution of (5.25)-(5.28) satisfying (7.1), (7.2), and properties (i)-(ii) of Proposition 7.1.

The existence of a unique solution $u_{R} \in C^{1, \alpha}\left(\overline{\Omega_{R-10 r}^{+}}\right) \cap H^{1}\left(\Omega_{R}^{+}\right)$of $(6.1)-(6.4)$ for any $R>L$ follows from Proposition 6.7.

Now, let $R_{j} \rightarrow \infty$. Using estimates (6.9) and (6.17) for $u_{R}$, which hold by Proposition 6.7, we can extract from $\left\{u_{R_{j}}\right\}$ a subsequence converging in $C^{1, \alpha / 2}$ on compact subsets of $\bar{\Omega}$ to a function $u \in C^{1, \alpha}(\bar{\Omega})$ that satisfies (7.1). Indeed, this can be achieved by extracting a subsequence converging in $C^{1, \alpha / 2}\left(\overline{\Omega_{L+1}^{+}}\right)$, a further subsequence converging in $C^{1, \alpha / 2}\left(\overline{\Omega_{L+2}^{+}}\right)$, etc., and by using the diagonal procedure. Obviously, $u$ is a weak solution of (5.25)-(5.27), since we can pass to the limit in (5.29) for any fixed $w \in C_{c}^{1}\left(\mathbf{R}^{n} \cap \overline{\Omega^{+}}\right)$.

Moreover, this solution $u$ satisfies property (ii): Indeed, for fixed $R_{0} \geq 1, R_{j} \geq R_{0}$ for sufficiently large $j$. For such $j$, by Lemma 6.4 , there exists $x_{j}^{\prime} \in \bar{\Lambda}$ such that $u_{R_{j}}\left(x_{j}^{\prime}, R_{0}\right)=0$. Then there exists a subsequence of $\left\{x_{j}^{\prime}\right\}$ converging to some point $x^{\prime} \in \bar{\Lambda}$. Since $u_{R_{j}}\left(\cdot, R_{0}\right) \rightarrow$ $u\left(\cdot, R_{0}\right)$ uniformly in $\bar{\Lambda}$, we have $u\left(x^{\prime}, R_{0}\right)=0$. Thus, the solution $u$ satisfies (i) and (ii).

Also, since each $u_{R}$ satisfies (6.9) and (6.17) with $C$ independent of $R$, then (7.1) and (7.2) hold.

Now we prove that $u$ satisfies (5.28). From (7.1), for any $\varepsilon>0$, there exists $\rho>L$ such that

$$
\|D u\|_{L^{2}\left(\Omega^{+} \cap\left\{x_{n}>\rho\right\}\right)} \leq \varepsilon .
$$

Then, by Proposition 6.5 (which holds for $u$ as $u_{R}$ ),

$$
\|D u\|_{0, \alpha, \Omega^{+} \cap\left\{x_{n}>\rho+1\right\}} \leq C \varepsilon .
$$

From this estimate and property (ii) proved above, we have

$$
\|u\|_{C^{0}\left(\Omega^{+} \cap\left\{x_{n}>\rho+1\right\}\right)} \leq C \varepsilon .
$$

Now (5.28) is proved.

7.2. Iteration Map. We first define an extension operator $\mathcal{P}_{\psi}$, similar to [4, Proposition 4.5] and [5, Proposition 5.1], to obtain

Proposition 7.2. Let $\sigma>0$ be sufficiently small and $\varphi^{-}$satisfy (4.5). Then, for any $\psi \in \mathcal{K}_{M}$, there exists an extension operator $\mathcal{P}_{\psi}: C^{1, \alpha}\left(\overline{\Omega^{+}(\psi)}\right) \rightarrow C^{1, \alpha}(\bar{\Omega})$ satisfying the following two properties:

(i) There exists $C$ depending only on $n, \gamma, q_{0}^{+}$, and $\alpha$ (but independent of $M, R, \sigma$, and $\psi$ ) such that, for the solution $u \in C^{1, \alpha}\left(\overline{\Omega^{+}(\psi)}\right)$ of problem (5.25)-(5.28) and $\varphi=u+q^{+} x_{n}$,

$$
\left\|\mathcal{P}_{\psi} \varphi-q^{+} x_{n}\right\|_{1, \alpha, \Omega} \leq C \sigma ;
$$

(ii) Let $\beta \in(0, \alpha)$. Let a sequence $\psi_{k} \in \mathcal{K}_{M}$ converges to $\psi \in C^{1, \beta}(\bar{\Omega})$ in the norm $\|\cdot\|_{1, \beta, \Omega}^{(-1)}$ defined in (5.2). Then $\psi \in \mathcal{K}_{M}$. Let $u_{k} \in C^{1, \alpha}\left(\overline{\Omega^{+}\left(\psi_{k}\right)}\right)$ and $u \in C^{1, \alpha}\left(\overline{\Omega^{+}(\psi)}\right)$ be the solutions of problems (5.25)-(5.28) for $\psi_{k}$ and $\psi$, respectively, and let $\varphi_{k}=u_{k}+q^{+} x_{n}$ and $\varphi=u+q^{+} x_{n}$. Then $\mathcal{P}_{\psi_{k}} \varphi_{k} \rightarrow \mathcal{P}_{\psi} \varphi$ in the norm $\|\cdot\|_{1, \beta, \Omega}^{(-1)}$.

Proof. Let $\psi \in \mathcal{K}_{M}$. We first define an appropriate extension operator $\mathcal{P}_{\psi}: C^{1, \alpha}\left(\overline{\Omega^{+}(\psi)}\right) \rightarrow$ $C^{1, \alpha}(\bar{\Omega})$.

Let $\varphi \in C^{1, \alpha}\left(\overline{\Omega^{+}\left(\psi_{j}\right)}\right)$ and $u=\varphi-q^{+} x_{n}$. Then $v:=u \circ \tilde{\Phi}^{-1}$ satisfies $v \in C^{1, \alpha}\left(\overline{\mathcal{C}_{(0, \infty)}}\right)$ with $\mathcal{C}_{(a, b)}=\Lambda \times(a, b)$, where $\tilde{\Phi}$ is defined by $(6.21)$.

We first define the extension operator $\mathcal{E}_{1}: C^{1, \beta}\left(\overline{\mathcal{C}_{(0, \infty)}}\right) \rightarrow C^{1, \beta}\left(\overline{\mathcal{C}_{(-2, \infty)}}\right)$ for any $\beta \in(0,1)$. Let $v \in C^{1, \beta}\left(\overline{\mathcal{C}_{(0, \infty)}}\right)$. Define $\mathcal{E}_{1} v=v$ in $\mathcal{C}_{(0, \infty)}$. For $\left(x^{\prime}, x_{n}\right) \in \mathcal{C}_{(-1,0)}$, define

$$
\mathcal{E}_{1} v\left(x^{\prime}, x_{n}\right)=\sum_{i=1}^{2} c_{i} v\left(x^{\prime},-\frac{x_{n}}{i}\right),
$$


where $c_{1}=-3$ and $c_{2}=4$, which are determined by $\sum_{i=1}^{2} c_{i}\left(-\frac{1}{i}\right)^{m}=1$ for $m=0,1$. Note that, by (6.22) and for sufficiently small $\sigma$, we have $\Omega \subset \tilde{\Phi}\left(\mathcal{C}_{(-2, \infty)}\right)$. Thus, $\left(\mathcal{E}_{1} v\right) \circ \tilde{\Phi} \in C^{1, \alpha}(\bar{\Omega})$.

Finally, we define

$$
\mathcal{P}_{\psi}(\varphi)(x)=\left(\mathcal{E}_{1} v\right) \circ \tilde{\Phi}(x)+q^{+} x_{n} .
$$

Obviously, $\mathcal{P}_{\psi}(\varphi) \in C^{1, \alpha}(\bar{\Omega})$. Also, since $\tilde{\Phi}\left(\Omega^{+}(\psi)\right)=\mathcal{C}_{(0, \infty)}$, we have

$$
\mathcal{P}_{\psi}(\varphi)(x)=v \circ \tilde{\Phi}(x)+q^{+} x_{n}=u(x)+q^{+} x_{n}=\varphi(x) \quad \text { for } x \in \Omega^{+}(\psi),
$$

i.e., $\mathcal{P}_{\psi}: C^{1, \alpha}\left(\overline{\Omega^{+}(\psi)}\right) \rightarrow C^{1, \alpha}(\bar{\Omega})$ is an extension operator.

Now, using (6.22), the following estimate is obtained as in the proof of [4, Proposition 4.5]:

$$
\left\|\mathcal{P}_{\psi} \varphi-q^{+} x_{n}\right\|_{1, \alpha, \Omega} \leq C\left\|\varphi-q^{+} x_{n}\right\|_{1, \alpha, \Omega^{+}(\psi)} .
$$

Then, if $u$ and $\varphi$ are those defined in (i), we have (7.2) for $u=\varphi-q^{+} x_{n}$ by Proposition 7.1, and thus (7.5) implies (7.4). The assertion (i) is proved.

Now we prove assertion (ii) of the proposition. First, by Lemma 5.1, $\psi \in \mathcal{K}_{M}$.

By (7.4) applied to $\psi_{j}$ and by Lemma 5.1, there exists a subsequence (still denoted by) $\varphi_{j}(x)$ such that

$$
\mathcal{P}_{\psi_{j}} \varphi_{j} \rightarrow \tilde{\varphi} \quad \text { in } \quad C_{(-1)}^{1, \beta}(\bar{\Omega})
$$

for some $\tilde{\varphi} \in C^{2, \alpha}(\bar{\Omega})$.

Denote $\tilde{\psi}_{k}:=\psi_{k} \circ \Psi$ and $\tilde{\psi}:=\psi \circ \Psi$. Then, by $(2.16), \tilde{\psi}_{k} \rightarrow \tilde{\psi}$ in $C^{1, \beta}(\bar{\Lambda} \times[-1,1])$. Now denote by $\tilde{f}_{k}\left(x^{\prime}\right)$ the function from (6.20) for $\psi_{k}(x)$. Then, by $(2.16),(4.5)$, and (5.4), $\tilde{f}_{k} \rightarrow \tilde{f}$ in $C^{1, \beta}(\bar{\Lambda})$. Now let $\tilde{\Phi}_{k}$ and $\tilde{\Phi}$ be the maps from $(6.21)$ for $\tilde{f}_{k}$ and $\tilde{f}$, respectively. Then $\tilde{\Phi}_{k}$ and $\tilde{\Phi}$ and their inverse maps satisfy (6.22), and $\tilde{\Phi}_{k} \rightarrow \tilde{\Phi}$ and $\tilde{\Phi}_{k}^{-1} \rightarrow \tilde{\Phi}^{-1}$ in $C^{1, \beta}$ on compact subsets of $\mathbf{R}^{n}$. Note $v_{k}:=u_{k} \circ \tilde{\Phi}_{k}^{-1} \in C^{1, \alpha}(\bar{\Lambda} \times[0, \infty))$. Recall that $u_{k}$ is a weak solution of (5.25)-(5.28) in the domain $\Omega^{+}\left(\psi_{k}\right)$, with coefficients $a_{i j}^{\left(\psi_{k}\right)}$ defined by (5.22) for $\psi_{k}$ and the corresponding right-hand sides (5.18) and (5.27) for $\psi_{k}$. Then, by Proposition 7.1, each $u_{k}$ satisfies (7.1) and (7.2). Then

$$
\begin{aligned}
& \left\|D v_{k}\right\|_{L^{2}(\Lambda \times(0, \infty))} \leq C \sigma \\
& \left\|v_{k}\right\|_{1, \alpha, \Lambda \times(0, \infty)} \leq C \sigma \quad \text { for } \quad k=1, \ldots
\end{aligned}
$$

Moreover, $v_{k}$ is a weak solution of a conormal problem

$$
\begin{array}{ll}
\sum_{i, j=1}^{n}\left(b_{i j}^{\left(\psi_{k}\right)} \partial_{x_{j}} v_{k}\right)_{x_{i}}=0 & \text { in } \quad \Lambda \times(0, \infty), \\
\sum_{i, j=1}^{n} b_{i j}^{\left(\psi_{k}\right)} \partial_{x_{j}} v_{k} \nu_{i}=\tilde{g}_{\psi_{k}}(x) & \text { on } \Lambda \times\{0\}, \\
\sum_{i, j=1}^{n} b_{i j}^{\left(\psi_{k}\right)} \partial_{x_{j}} v_{k} \nu_{i}=h_{\psi_{k}}(x) & \text { on } \quad \partial \Lambda \times(0, \infty),
\end{array}
$$

where $b_{i j}^{\left(\psi_{k}\right)}, \tilde{g}_{\psi_{k}}$, and $h_{\psi_{k}}$ are defined by the coefficients and right-hand sides of (5.25)-(5.27) for $\psi_{k}$ and by $\tilde{\Phi}_{k}^{-1}$. In particular, since $a_{i j}^{\left(\psi_{k}\right)} \rightarrow a_{i j}^{(\psi)}$ in $C^{1, \beta}$ on compact subsets of $\bar{\Omega}$ as $k \rightarrow \infty$, which follows from (5.22), and since $\tilde{\Phi}_{k}^{-1} \rightarrow \tilde{\Phi}^{-1}$ in $C^{1, \beta}$ on compact subsets of $\mathbf{R}^{n}$ ), it is easy to show that, for any compact $K \subset \bar{\Lambda} \times[0, \infty)$,

$$
b_{i j}^{\left(\psi_{k}\right)} \rightarrow b_{i j}^{(\psi)} \text { in } C^{1, \beta}(K) \quad \text { and } \quad\left(\tilde{g}_{\psi_{k}}, h_{\psi_{k}}\right) \rightarrow\left(\tilde{g}_{\psi}, h_{\psi}\right) \text { in } C^{\beta}(K),
$$

where $b_{i j}^{(\psi)}, \tilde{g}_{\psi}$, and $h_{\psi}$ are the coefficients and right-hand sides of (7.9)-(7.11) for $\psi$. By (7.8), there exists a subsequence $v_{k_{m}}$ which converges in $C^{1, \beta}$ on the compact subsets of $\bar{\Lambda} \times[0, \infty)$ to 
some $v \in C^{1, \beta}(\bar{\Lambda} \times[0, \infty))$. Then, choosing $w \in C_{c}^{1}\left(\mathbf{R}^{n}\right)$ in the weak formulation (5.29) for the conormal problem (7.9)-(7.11):

$$
\int_{\Lambda \times(0, \infty)} \sum_{i, j=1}^{n} b_{i j}^{\left(\psi_{k}\right)} v_{x_{k}} w_{x_{i}} d x+\int_{\Lambda \times\{0\}} \tilde{g}_{\psi_{k}} w d x^{\prime}-\int_{\partial \Lambda \times(0, \infty)} h_{\psi_{k}} w d \mathcal{H}^{n-1}=0 .
$$

We pass to the limit for the subsequence $\left\{k_{m}\right\}$ and conclude that $v$ is a weak solution of the problem (7.9)-(7.11) with coefficients $b_{i j}^{(\psi)}$ and right-hand sides $\tilde{g}_{\psi}$ and $h_{\psi}$. Note also that $v$ satisfies (7.7) since each $v_{k_{m}}$ satisfies (7.7). Transforming back, we conclude that $\tilde{u}:=v \circ \tilde{\Phi}$ is a weak solution of the problem (5.25)-(5.27) for $\psi$, and $\tilde{u}$ satisfies (7.1). Now the proof of Proposition 7.1 implies that $\tilde{u}$ satisfies (5.28). Then the uniqueness assertion of Proposition 7.1 implies $u=\tilde{u}$ on $\Omega^{+}(\psi)$. Thus

$$
\mathcal{P}_{\psi}(\varphi)=\left(\mathcal{E}_{1} v\right) \circ \tilde{\Phi}+q^{+} x_{n}
$$

Finally,

$$
\mathcal{P}_{\psi_{k_{m}}}\left(\varphi_{k_{m}}\right)=\left(\mathcal{E}_{1} v_{k_{m}}\right) \circ \tilde{\Phi}_{k_{m}}+q^{+} x_{n} \rightarrow\left(\mathcal{E}_{1} v\right) \circ \tilde{\Phi}+q^{+} x_{n} \quad \text { in } C^{1, \beta}(K)
$$

for any compact $K \subset \bar{\Omega}$, since $\tilde{\Phi}_{k} \rightarrow \tilde{\Phi}, v_{k_{m}} \rightarrow v$ in $C^{1, \beta}(K)$, and $\mathcal{E}_{1}: C^{1, \beta}\left(\overline{\mathcal{C}_{(0, \infty)}}\right) \rightarrow$ $C^{1, \beta}\left(\overline{\mathcal{C}_{(-2, \infty)}}\right)$ is continuous. From this, using (7.12) and (7.6), we get $\mathcal{P}_{\psi_{k_{m}}}\left(\varphi_{k_{m}}\right) \rightarrow \mathcal{P}_{\psi}(\varphi)$ in $C_{(-1)}^{1, \beta}(\bar{\Omega})$. Moreover, by the same argument, from any subsequence of $\mathcal{P}_{\psi_{k}}\left(\varphi_{k}\right)$, we can extract a further subsequence converging in $C_{(-1)}^{1, \beta}(\bar{\Omega})$ to the same limit $\mathcal{P}_{\psi}(\varphi)$. Thus the whole sequence $\mathcal{P}_{\psi_{k}}\left(\varphi_{k}\right)$ converges to this limit. Proposition 7.2 is proved.

Now we can define the iteration map $J: \mathcal{K}_{M} \rightarrow C^{1, \alpha}(\bar{\Omega})$ by

$$
J \psi:=\mathcal{P}_{\psi} \varphi
$$

with $\varphi(x)=u(x)+q^{+} x_{n}$, where $u \in C^{1, \alpha}\left(\overline{\Omega^{+}(\psi)}\right)$ is the unique solution of problem (5.25)-(5.28) with $\psi \in \mathcal{K}_{M}$. By Proposition $7.2(\mathrm{ii}), J$ is continuous in the $C^{1, \beta}(\bar{\Omega})$-norm for any positive $\beta<\alpha$.

7.3. Existence of Solutions of the Free Boundary Problem. Now we can prove the existence of solutions of the free boundary problem. We denote by $\varphi$ both the function $\varphi$ in $\Omega^{+}(\psi)$ and its extension $\mathcal{P}_{\psi} \varphi$.

Choose $M$ to be the constant $C$ from (7.4). Then, for $\psi \in \mathcal{K}_{M}$, we have from Proposition 7.2(i) that $\varphi:=J \psi \in \mathcal{K}_{M}$ if $\sigma>0$ is sufficiently small and depends only on $n, \gamma, q^{+}$, and $\Omega$, since $M>0$ is now fixed. Thus, (7.13) defines the iteration map $J: \mathcal{K}_{M} \rightarrow \mathcal{K}_{M}$ and, from Proposition 7.2(ii), $J$ is continuous on $\mathcal{K}_{M}$ in the $C_{(-1)}^{1, \alpha / 2}(\bar{\Omega})$-norm.

In order to find a solution of Problem (TFB), we seek a fixed point of the map $J$. We use the Schauder Fixed Point Theorem (cf. [21, Theorem 11.1]) in the following setting:

Let $\sigma>0$ satisfy the conditions of Proposition 7.2. By Lemma 5.1, the set $\mathcal{K}_{M}$ is a compact convex subset of $C_{(-1)}^{1, \alpha / 2}(\bar{\Omega})$. We have shown that $J\left(\mathcal{K}_{M}\right) \subset \mathcal{K}_{M}$ and $J$ is continuous in the $C_{(-1)}^{1, \alpha / 2}(\bar{\Omega})$-norm. Then, by the Schauder Fixed Point Theorem, $J$ has a fixed point $\varphi \in \mathcal{K}_{M}$.

If $\varphi$ is such a fixed point, then

$$
\tilde{\varphi}(x):=\min \left(\varphi^{-}(x), \varphi(x)\right)
$$

is a classical solution of Problem (TFB) and $S_{\varphi}$ is its free boundary.

It follows that $\tilde{\varphi}$ is a solution of Problem (FB), thus Problem (TN), provided that $\sigma$ is small enough so that (7.4) implies that $|D \varphi(x)|<c_{*}-\varepsilon$, where $\varepsilon=\left(c_{*}-q_{0}^{+}\right) / 2$. Indeed, then (4.1) implies that $\varphi$ lies in the untruncated region for equation (4.4). Also, $|D \tilde{\varphi}|<c_{*}-\varepsilon$ on $\Omega^{+}(\tilde{\varphi}):=\left\{\tilde{\varphi}(x)<\varphi^{-}(x)\right\}$ and $|D \tilde{\varphi}|>c_{*}$ on $\Omega \backslash \Omega^{+}$since $\tilde{\varphi}=\varphi$ on $\Omega^{+}(\tilde{\varphi})$ and $\tilde{\varphi}=\varphi^{-}$on $\Omega \backslash \Omega^{+}$. 


\section{UNIQUENESS AND STABILITY}

We now prove the uniqueness and stability of solutions of Problem (TN) when $\varphi^{-} \in C^{2, \alpha}$. We first prove the following lemma.

Lemma 8.1. Let $\varphi^{-}$satisfy (4.5). Let $\varphi \in C^{0,1}(\Omega)$ be a solution of Problem $(\mathrm{TN})$ with $\omega \in\left(0, c_{*}\right)$ satisfying (2.24). Then there exists $\sigma_{0}>0$ depending only on $n, \alpha, \gamma$, and $q_{0}^{+}$such that, if $\sigma \in\left(0, \sigma_{0}\right), \varphi$ satisfies $(2.23),(2.30)$, and

$$
\left\|D \varphi-\omega e_{n}\right\|_{C^{0}\left(\Omega \cap\left\{x_{n}>R\right\}\right)} \rightarrow 0 \quad \text { as } R \rightarrow \infty .
$$

Proof. The proof that $\varphi$ satisfies $(2.23)$ and (2.30) is the same as in [5, Lemma 7.1].

Now we prove (8.1). Setting $w(x):=\varphi(x)-\omega x_{n}$ and using (2.17), (2.23), and (2.30), we see that $w$ satisfies

$$
\begin{aligned}
& \sum_{i, j=1}^{n}\left(a_{i j} w_{x_{j}}\right)_{x_{i}}=0 \quad \text { in } \Omega \cap\left\{x_{n}>L\right\}, \\
& \sum_{i, j=1}^{n} a_{i j} w_{x_{j}} \nu_{i}=-\tilde{\rho}\left(\left(q^{+}\right)^{2}\right) q^{+} \nu \cdot e_{n} \quad \text { on } \quad \partial_{l} \Omega^{+} \cap\left\{x_{n}>L\right\}, \\
& \lim _{R \rightarrow \infty}\|w\|_{\left.C(\Omega) \cap\left\{x_{n}>R\right\}\right)}=0,
\end{aligned}
$$

where

$$
\begin{aligned}
a_{i j}(x)= & \int_{0}^{1}\left\{\tilde{\rho}\left(\left|t D \varphi(x)+(1-t) \omega e_{n}\right|^{2}\right) \delta_{i}^{j}\right. \\
& \left.+2 \tilde{\rho}^{\prime}\left(\left|t D \varphi(x)+(1-t) \omega e_{n}\right|^{2}\right)\left(t \varphi_{x_{i}}(x)+(1-t) \omega \delta_{i}^{n}\right)\left(t \varphi_{x_{j}}(x)+(1-t) \omega \delta_{j}^{n}\right)\right\} d t .
\end{aligned}
$$

By (2.24), the equation is elliptic if $\sigma$ is small. Then the proof of (8.1) is similar to the proof of (7.3) in Proposition 7.1.

Now we show that $\omega$ in Problem $(\mathrm{TN})$ is uniquely determined by $\varphi^{-}$.

Proposition 8.2. There exists $\sigma_{0}$ depending only on the data as in Lemma 8.1 such that, if $\varphi^{-}$ satisfies (4.5) and $\varphi \in C^{0,1}(\Omega)$ is a solution of Problem $(\mathrm{TN})$ with $\omega \in\left(0, c_{*}\right)$ satisfying (2.24), then $\omega=q^{+}$in $(2.20)$, with $q^{+}$defined by $(2.26)$ and $\varphi(x)$ satisfies $(2.28)$.

This can be achieved by following the proof of [5, Proposition 7.2] and using Lemma 8.1 instead of [5, Lemma 7.1].

It remains to prove the uniqueness of solutions of Problem (TN) satisfying (2.24).

Proposition 8.3. There exists $\sigma_{0}$ depending only on the data as in Lemma 8.1 such that, if $\varphi^{-}$ satisfies (4.5), then the solution $\varphi \in C^{0,1}(\Omega)$ of Problem $(\mathrm{TN})$ with $\omega=q^{+}$satisfying (2.24) is unique.

The rest of this section is to prove Proposition 8.3 by using a version of the partial hodograph transform developed in [6].

8.1. Extension of $\varphi^{-}$to the Domain $\Omega$. Since $\varphi^{-}$satisfies (2.19) and (4.5) in the domain $\Omega_{1}:=\Omega \cap\left\{x_{n}<1\right\}$, then we can extend $\varphi^{-}$to $\Omega$ so that the extension (still denoted by) $\varphi^{-}$is in $C^{2, \alpha}(\Omega)$, coincides with the original $\varphi^{-}$in $\Omega \cap\left\{x_{n}<1 / 2\right\}$, and

$$
\begin{aligned}
& \left\|\varphi^{-}-\varphi_{0}^{-}\right\|_{2, \alpha, \Omega} \leq C(n, \alpha) \sigma \\
& \operatorname{supp}\left(\varphi^{-}-\varphi_{0}^{-}\right) \subset \Omega \cap\left\{x_{n}<L\right\} \\
& \partial_{\nu} \varphi^{-}=0 \quad \text { on } \partial_{l} \Omega
\end{aligned}
$$


where $\varphi_{0}^{-}(x)=q_{0}^{-} x_{n}$. To construct such an extension, we first note that we can modify the mapping $\Psi$ and replace it by a mapping $\Psi_{1} \in C^{2, \alpha}\left(\mathbf{R}^{n}, \mathbf{R}^{n}\right)$ such that $\Psi_{1}$ satisfies (2.14), $\left\|\Psi_{1}-I d\right\|_{2, \alpha, \mathbf{R}^{n}} \leq C \sigma,(2.17)$, and

$$
D \Psi_{1}(x) \nu_{\partial \mathcal{C}}(x)=\zeta(x) \nu_{\partial_{l} \Omega}\left(\Psi_{1}(x)\right) \quad \text { for any } x \in \partial \mathcal{C},
$$

where $\mathcal{C}=\Lambda \times(-\infty, \infty), \nu_{\partial \mathcal{C}}(\cdot)$ and $\nu_{\partial_{l} \Omega}(\cdot)$ are the inward unit normals to $\partial \mathcal{C}$ and $\partial_{l} \Omega$ respectively, and $\|\zeta-1\|_{2, \alpha, \partial \mathcal{C}} \leq C \sigma$. To construct such $\Psi_{1}$, we first define the corresponding $C^{2, \alpha}$ mapping $\tilde{\Psi}_{1}$ between sufficiently small neighborhoods (depending only on $\Lambda$ and $\sigma$ ) of $\partial \mathcal{C}$ and $\partial_{l} \Omega$ by

$$
\tilde{\Psi}_{1}\left(z+s \nu_{\partial \mathcal{C}}(z)\right)=\Psi(z)+s \nu_{\partial_{l} \Omega}(\Psi(z))
$$

for $z \in \partial \mathcal{C}$ and $s \in(0, \varepsilon)$, where $\varepsilon>0$ depends only on $\Lambda$ and $L$. Then we let

$$
\Psi_{1}(x)=\eta_{\varepsilon}\left(d_{\partial_{l} \Omega}(x)\right) \tilde{\Psi}_{1}(x)+\left(1-\eta_{\varepsilon}\left(d_{\partial_{l} \Omega}(x)\right)\right) \Psi(x),
$$

where $d_{\partial_{l} \Omega}(\cdot)$ is the distance function to $\partial_{l} \Omega$ and $\eta_{\varepsilon}: \mathbf{R} \rightarrow \mathbf{R}$ is a smooth function satisfying $0 \leq \eta_{\varepsilon} \leq 1$ on $\mathbf{R}, \eta_{\varepsilon} \equiv 1$ on $[-\infty, 7 \varepsilon / 8)$, and $\eta_{\varepsilon} \equiv 0$ on $(\varepsilon, \infty)$. Now we define $\tilde{\varphi}^{-}(x)=\varphi^{-}\left(\Psi_{1}(x)\right)$ on $\Psi_{1}^{-1}\left(\Omega_{1}\right)$. Then, if $\sigma$ is small, $\tilde{\varphi}^{-}$is defined at least on $\Lambda \times(-7 / 8,7 / 8)$ and satisfies $D \tilde{\varphi}^{-} \cdot \nu_{\mathcal{C}}=0$ on $\partial \Lambda \times(-7 / 8,7 / 8)$. Now we define

$$
\hat{\varphi}^{-}(x)=\eta_{7 / 8}\left(x_{n}\right) \tilde{\varphi}^{-}(x)+\left(1-\eta_{7 / 8}\left(x_{n}\right)\right) q^{-} x_{n} \quad \text { on } \Lambda \times(-7 / 8, \infty),
$$

and finally the function $\varphi^{-}(x)=\hat{\varphi}^{-}\left(\Psi_{1}^{-1}(x)\right)$ is a $C^{2, \alpha}$ extension of $\varphi^{-}$satisfying (8.2)-(8.4).

Denote

$$
g=\operatorname{div}\left(\rho\left(\left|D \varphi^{-}\right|^{2}\right) D \varphi^{-}\right) \quad \text { in } \Omega .
$$

Since $\varphi^{-}$satisfies (1.1) in $\Omega_{1}$, then, from (8.2)-(8.3), we have that $g$ satisfies

$$
\begin{aligned}
& g \in C^{\alpha}(\bar{\Omega}), \quad\|g\|_{0, \alpha, \Omega} \leq C \sigma, \\
& g \equiv 0 \quad \text { on } \quad\left(\Omega \cap\left\{x_{n}<1\right\}\right) \cup\left(\Omega \cap\left\{x_{n}>L\right\}\right) .
\end{aligned}
$$

From now on, we use the extended function $\varphi^{-}=\varphi^{-}(x)$, and $C$ may denote a different constant at each occurrence, depending only on the data, unless otherwise is specified.

8.2. Partial Hodograph Transform. Let $\varphi$ be a solution of Problem (TN) with $\omega=q^{+}$and satisfy (2.24). Then $\Omega^{+}:=\left\{|D \varphi|<c_{*}\right\}$ has the form (2.23) and (2.30). Define a function $u$ in $\Omega^{+}$by

$$
u(x):=\varphi^{-}(x)-\varphi(x) .
$$

Then (2.31) and (8.2) imply

$$
\left\|u-\left(q_{0}^{-}-q^{+}\right) x_{n}\right\|_{1, \alpha, \Omega^{+}} \leq C \sigma .
$$

In particular, if $\sigma$ is sufficiently small, we use (2.29) to get

$$
0<\left(q_{0}^{-}-q_{0}^{+}\right) / 2 \leq u_{x_{n}}(x) \leq 2\left(q_{0}^{-}-q_{0}^{+}\right) \quad \text { for any } \quad x \in \Omega^{+} .
$$

Furthermore, (2.28) and (8.3) imply

$$
\left\|u-\left(q_{0}^{-}-q^{+}\right) x_{n}\right\|_{C^{1}\left(\Omega \cap\left\{x_{n}>R\right\}\right)} \rightarrow 0 \quad \text { as } R \rightarrow \infty .
$$

From (2.24) with sufficiently small $\sigma, \varphi$ satisfies (1.1) in $\Omega^{+}$and (2.4) on $S$. Then, since $S \subset \Omega_{1}$ and $\varphi^{-}$satisfies (1.1) in $\Omega_{1}$ and (8.4) on $\partial_{l} \Omega$, we see that $u$ is a solution of the following problem:

$$
\begin{array}{ll}
\operatorname{div}(A(x, D u))=-g & \text { in } \Omega^{+}, \\
A(x, D u) \cdot \nu=0 & \text { on } S, \\
A(x, D u) \cdot \nu=0 & \text { on } \partial_{l} \Omega^{+},
\end{array}
$$

where, for $x \in \Omega^{+}$and $P \in \mathbf{R}^{n}$,

$$
A(x, P)=\rho\left(\left|D \varphi^{-}(x)-P\right|^{2}\right)\left(D \varphi^{-}(x)-P\right)-\rho\left(\left|D \varphi^{-}(x)\right|^{2}\right) D \varphi^{-}(x) .
$$


The equation in (8.10) is uniformly elliptic for $u$ if $\sigma$ is sufficiently small, which follows from (4.5) and (8.7), since

$$
0<c_{0} \leq \Phi^{\prime}(q)=\rho\left(q^{2}\right)+2 q^{2} \rho^{\prime}\left(q^{2}\right) \leq C \quad \text { for } q \text { near } q_{0}^{+},
$$

for some constants $c_{0}$ and $C>0$. Note that $u$ satisfies the weak form of problem (8.10):

$$
\int_{\Omega^{+}}(A(x, D u) \cdot D \eta-g \eta) d x=0 \quad \text { for any } \eta \in C_{0}^{1}\left(\mathbf{R}^{n}\right) .
$$

Since $\varphi=\varphi^{-}$on $S$, it follows that

$$
u=0 \quad \text { on } \mathrm{S} \text {. }
$$

Now we make a change of variables $z=\Phi(x)$ to rewrite problem (8.12) into a cylindric domain $\mathcal{C}^{+}:=\Phi\left(\Omega^{+}\right)$, where $\Psi$ and its inverse $\Phi$ are the maps in (2.14), (2.16), and (6.18). It follows that, if $\sigma$ is sufficiently small, then

$$
\mathcal{C}^{+}=(\Lambda \times(-\infty, \infty)) \cap\left\{z_{n}>\tilde{f}\left(z^{\prime}\right)\right\}, \quad\|\tilde{f}\|_{1, \alpha, \mathbf{R}^{n-1}} \leq C M \sigma,
$$

and $\partial \mathcal{C}^{+}=\overline{\partial_{l} \mathcal{C}^{+}} \cup \tilde{S}$, where

$$
\begin{aligned}
& \partial_{l} \mathcal{C}^{+}:=(\partial \Lambda \times(-\infty, \infty)) \cap\left\{z_{n}>\tilde{f}\left(z^{\prime}\right)\right\}=\Phi\left(\partial_{l} \Omega^{+}\right), \\
& \tilde{S}:=(\Lambda \times(-\infty, \infty)) \cap\left\{z_{n}=\tilde{f}\left(z^{\prime}\right)\right\}=\Phi(S) .
\end{aligned}
$$

Also, $\Omega^{+}=\Psi\left(\mathcal{C}^{+}\right)$. Consider the function

$$
\tilde{u}(z)=u(\Psi(z)) \quad \text { for } z \in \mathcal{C}^{+} .
$$

Then (2.16), (2.17), and (8.7) imply

$$
\left\|\tilde{u}-\left(q_{0}^{-}-q^{+}\right) x_{n}\right\|_{1, \alpha, \mathcal{C}^{+}} \leq C \sigma .
$$

In particular, $\tilde{u}$ satisfies (8.8) if $\sigma$ is small. By (8.9) and (2.17),

$$
\left\|\tilde{u}-\left(q_{0}^{-}-q^{+}\right) x_{n}\right\|_{C^{1}(\Lambda \times(R, \infty))} \rightarrow 0 \quad \text { as } R \rightarrow \infty .
$$

From (8.13), $\tilde{u}$ satisfies

$$
\tilde{u}=0 \quad \text { on } \mathrm{S} .
$$

Moreover, $\tilde{u}$ is a weak solution of a fixed boundary problem of the form (8.10) in the domain $\mathcal{C}^{+}$with modified functions $\tilde{A}(x, P)$ and $\tilde{g}(x)$ satisfying $\tilde{A} \in C^{1}\left(\overline{\Lambda \times(-1, \infty)} \times \mathbf{R}^{n}\right)$ and $\tilde{g} \in$ $C^{\alpha}(\overline{\Lambda \times(-1, \infty)})$. Precisely, $\tilde{u}$ satisfies

$$
\int_{\mathcal{C}^{+}}(\tilde{A}(x, D \tilde{u}) \cdot D \eta-\tilde{g} \eta) d x=0 \quad \text { for any } \eta \in C_{0}^{1}\left(\mathbf{R}^{n}\right) .
$$

This is obtained by using the test function $\eta \circ \Psi$, making the change of variables $x \rightarrow \Psi(x)$ in (8.12), and obtaining the expressions for $\tilde{A}$ in $\mathcal{C}^{+} \times \mathbf{R}^{n}$ and $\tilde{g}$ in $\mathcal{C}^{+}$in terms of $A, g$, and the map $\Psi$ which are well defined in $\Lambda \times(-1, \infty) \times \mathbf{R}^{n}$ and $\Lambda \times(-1, \infty)$, respectively. We do not write the explicit expressions of $\tilde{A}$ and $\tilde{g}$, but only point out some properties of $\tilde{A}$ and $\tilde{g}$, which can be obtained readily by using these expressions. First, we emphasize that $\tilde{A}$ and $\tilde{g}$ are given in terms of the original functions $A, g$, and the map $\Psi$, which are independent of a solution $\varphi$ of Problem (TN). Next, using (2.17), we see that, for any $R>0$, there exists $C_{R}>$ such that

$$
\left|D_{P} A(\Psi(x), P)-D_{P} \tilde{A}(x, P)\right| \leq C_{R}\|\Psi\|_{C^{1}\left(\mathbf{R}^{n}\right)} \quad \text { for any } x \in \mathcal{C}^{+}, P \in \mathbf{R}^{n},|P|<R .
$$

Furthermore, from (2.17), it follows that $A=\tilde{A}$ for $x_{n}>L$, i.e.,

$$
A(x, P)=\tilde{A}(x, P) \quad \text { for any } x \in \Lambda \times(L, \infty), P \in \mathbf{R}^{n} .
$$

Similarly, from (2.16), (2.17), and (8.6), we conclude that $\tilde{g}$ satisfies

$$
\begin{aligned}
& \tilde{g} \in C^{\alpha}(\bar{\Lambda} \times[-1, \infty)), \quad\|\tilde{g}\|_{0, \alpha, \Lambda \times(-1, \infty)} \leq C \sigma, \\
& \tilde{g} \equiv 0 \quad \text { on } \quad\left(\Omega \cap\left\{x_{n}<1 / 2\right\}\right) \cup\left(\Omega \cap\left\{x_{n}>L\right\}\right)
\end{aligned}
$$


if $\sigma$ is sufficiently small. Define

$$
\tilde{F}\left(x^{\prime}, x_{n}\right)=\int_{0}^{x_{n}} \tilde{g}\left(x^{\prime}, s\right) d s \quad \text { for }\left(x^{\prime}, x_{n}\right) \in \Lambda \times(-1, \infty) .
$$

Then, from (8.21) and (8.22), we have

$$
\begin{aligned}
& \tilde{F}, \tilde{F}_{x_{n}} \in C^{\alpha}(\bar{\Lambda} \times[-1, \infty)), \\
& \|\tilde{F}\|_{0, \alpha, \Lambda \times(-1, \infty)} \leq C \sigma, \quad\left\|\tilde{F}_{x_{n}}\right\|_{0, \alpha, \Lambda \times(-1, \infty)} \leq C \sigma, \\
& \tilde{F} \equiv 0 \quad \text { in } \Lambda \times(-1,1 / 2), \\
& \tilde{F} \text { is independent of } x_{n} \in \Lambda \times(L, \infty) .
\end{aligned}
$$

Note that, from (8.14), for sufficiently small $\sigma$, the free boundary $\tilde{S}$ lies within the domain $\Lambda \times(-1,1 / 2)$. Then, by (8.25), the function $\tilde{F}$ vanishes on $\tilde{S}$. Using also (8.14) and (8.23), we can rewrite $(8.18)$ as

$$
\int_{\mathcal{C}^{+}}\left(\tilde{A}(x, D \tilde{u})+\tilde{F}(x) e_{n}\right) \cdot D \eta d x=0 \quad \text { for any } \eta \in C_{0}^{1}\left(\mathbf{R}^{n}\right) .
$$

Thus, $\tilde{u}$ is a weak solution of the following conormal problem:

$$
\begin{array}{ll}
\operatorname{div}\left(\tilde{A}(x, D u)+\tilde{F} e_{n}\right)=0 & \text { in } \mathcal{C}^{+}, \\
\left(\tilde{A}(x, D u)+\tilde{F} e_{n}\right) \cdot \nu=0 & \text { on } \tilde{S}, \\
\left(\tilde{A}(x, D u)+\tilde{F} e_{n}\right) \cdot \nu=0 & \text { on } \partial_{l} \mathcal{C}^{+} .
\end{array}
$$

Now we make the partial hodograph transform. Define a mapping $\mathcal{F}: \overline{\mathcal{C}^{+}} \rightarrow \mathbf{R}^{n}$ by

$$
\left(x^{\prime}, x_{n}\right) \rightarrow\left(y^{\prime}, y_{n}\right)=\left(x^{\prime}, \tilde{u}\left(x^{\prime}, x_{n}\right)\right) .
$$

The nondegeneracy property (8.8) of $\tilde{u}$ implies that the mapping $\mathcal{F}$ is one-to-one on $\overline{\mathcal{C}^{+}}$and, from (8.8), (8.13), and (8.14),

$$
\mathcal{F}\left(\mathcal{C}^{+}\right)=\Lambda \times(0, \infty), \quad \mathcal{F}(\tilde{S})=\Lambda \times\{0\}
$$

i.e., the free boundary $\tilde{S}$ is mapped to the fixed boundary $\Lambda \times\{0\}$. Also, by (8.8) for $\tilde{u}$, there exists a function $v \in C^{1, \alpha}(\overline{\Lambda \times(0, \infty)})$ such that, for $\left(x^{\prime}, x_{n}\right) \in \overline{\mathcal{C}^{+}}$and $y_{n} \geq 0$,

$$
\tilde{u}\left(x^{\prime}, x_{n}\right)=y_{n} \quad \text { if and only if } \quad v\left(x^{\prime}, y_{n}\right)=x_{n} .
$$

Thus,

$$
\mathcal{F}^{-1}\left(y^{\prime}, y_{n}\right)=\left(y^{\prime}, v\left(y^{\prime}, y_{n}\right)\right) .
$$

Differentiating the identity $\tilde{u}\left(x^{\prime}, v\left(x^{\prime}, y_{n}\right)\right)=y_{n}$, which holds for any $\left(x^{\prime}, y_{n}\right) \in \overline{\Lambda \times(0, \infty)}$, we find

$$
v_{y_{n}}>0 \quad \text { in } \Lambda \times(0, \infty)
$$

and

$$
D_{x^{\prime}} u=-\frac{1}{v_{y_{n}}} D_{y^{\prime}} v, \quad u_{x_{n}}=\frac{1}{v_{y_{n}}},
$$

where the left-hand and right-hand sides are taken at the points $\left(x^{\prime}, x_{n}\right)$ and $\mathcal{F}\left(x^{\prime}, x_{n}\right)$, respectively. In particular, property (8.8) of $\tilde{u}$ implies

$$
0<\frac{1}{2\left(q_{0}^{-}-q_{0}^{+}\right)} \leq v_{y_{n}}(y) \leq \frac{2}{q_{0}^{-}-q_{0}^{+}} \quad \text { for any } \quad y \in \mathcal{C}^{+} .
$$

From this and (8.15), we get

$$
\left\|v-v_{0}\right\|_{1, \alpha, \Lambda \times(0, \infty)} \leq C \sigma
$$

where $v_{0}(y)=y_{n} /\left(q_{0}^{-}-q^{+}\right)$. From (8.16) and (8.33),

$$
\left\|v-v_{0}\right\|_{C^{1}(\Lambda \times(R, \infty))} \rightarrow 0 \quad \text { as } R \rightarrow \infty .
$$


Now, since $\tilde{u}(x)$ is a solution of the conormal problem (8.27) in $\mathcal{C}^{+}$, then $v(y)$ is a solution of the corresponding problem in $\Lambda \times(0, \infty)$. In order to show that this problem has also a conormal structure, we make the change of variables $x \rightarrow y=\mathcal{F}(x)$ in the weak form (8.27) of problem (8.28)-(8.29). In order to do that, we especially need to change the variables in the test function $\eta$. For that, we note that the function $\psi(y):=\eta \circ \mathcal{F}^{-1}(y)=\eta\left(y^{\prime}, v\left(y^{\prime}, y_{n}\right)\right)$ satisfies $\psi \in C^{1}(\overline{\Lambda \times(0, \infty)})$ and, if $\eta \equiv 0$ on $\mathbf{R}^{n} \backslash B_{R}$, then $\psi \equiv 0$ on $(\Lambda \times(0, \infty)) \backslash B_{R_{1}}$ for some $R_{1}$, i.e., $\psi=\left.\tilde{\psi}\right|_{\Lambda \times(0, \infty)}$ for some $\tilde{\psi} \in C_{0}^{1}\left(\mathbf{R}^{n}\right)$. Similarly, for any $\psi \in C_{0}^{1}\left(\mathbf{R}^{n}\right)$, there exists $\eta \in C_{0}^{1}\left(\mathbf{R}^{n}\right)$ such that $\left.\psi\right|_{\overline{\mathbf{R}_{+}^{n}}}=\eta \circ \mathcal{F}^{-1}$ and $\eta(x)=\psi \circ \mathcal{F}(x)$ for $x \in \overline{\mathcal{C}^{+}}$. We differentiate the identity $\eta(x)=\psi\left(x^{\prime}, u\left(x^{\prime}, x_{n}\right)\right)$ and use (8.32) to obtain

$$
D_{x^{\prime}} \eta=D_{y^{\prime}} \psi-\frac{\psi_{y_{n}}}{v_{y_{n}}} D_{y^{\prime}} v, \quad \eta_{y_{n}}=\frac{\psi_{y_{n}}}{v_{y_{n}}} .
$$

Now, in (8.27), we make the change of variables $x \rightarrow y=\mathcal{F}(x)$, use (8.32) and (8.36), note that the Jacobian of $\mathcal{F}^{-1}$ is $J \mathcal{F}^{-1}(y)=v_{y_{n}}(y)$, and write $\tilde{A}\left(x^{\prime}, x_{n}, p^{\prime}, p_{n}\right)$ for $\tilde{A}(x, P)$ and $\tilde{F}\left(x^{\prime}, x_{n}\right)$ for $\tilde{F}(x)$ to obtain

$$
\int_{\Lambda \times(0, \infty)}\left(B\left(y^{\prime}, v, D v\right)+\tilde{F}\left(y^{\prime}, v\right) e_{n}\right) \cdot D \psi d y=0 \quad \text { for any } \psi \in C_{0}^{1}\left(\mathbf{R}^{n}\right),
$$

where, for $y^{\prime} \in \Lambda, z \in \mathbf{R}, P=\left(p^{\prime}, p_{n}\right) \in \mathbf{R}^{n-1} \times \mathbf{R}_{+}$,

$$
\begin{aligned}
& B^{i}\left(y^{\prime}, z, P\right)=\tilde{A}^{i}\left(y^{\prime}, z,-\frac{p^{\prime}}{p_{n}}, \frac{1}{p_{n}}\right) p_{n} \quad \text { for } i=1, \ldots, n-1, \\
& B^{n}\left(y^{\prime}, z, P\right)=\tilde{A}^{n}\left(y^{\prime}, z,-\frac{p^{\prime}}{p_{n}}, \frac{1}{p_{n}}\right)-\sum_{1}^{n-1} \tilde{A}^{i}\left(y^{\prime}, z,-\frac{p^{\prime}}{p_{n}}, \frac{1}{p_{n}}\right) p_{i} .
\end{aligned}
$$

Thus, $v(y)$ is a weak solution of the corresponding conormal problem in $\Lambda \times(0, \infty)$.

Note that the conormal problem (8.37) is elliptic for the functions satisfying (8.34) with small $\sigma$, that is, there exists $\tilde{\lambda}>0$ such that

$$
\tilde{\lambda}|\xi|^{2} \leq \sum_{i, j=1}^{n} B_{p_{j}}^{i}\left(y^{\prime}, v(y), D v(y)\right) \xi_{i} \xi_{j} \leq \tilde{\lambda}^{-1}|\xi|^{2}
$$

for any $y \in \Lambda \times(0, \infty), \xi \in \mathbf{R}^{n}$, and $v$ satisfying (8.34). To see this, from (8.38), we compute

$$
\sum_{i, j=1}^{n} B_{p_{j}}^{i}\left(y^{\prime}, z, p\right) \xi_{i} \xi_{j}=\sum_{i, j=1}^{n} \tilde{A}_{p_{j}}^{i}\left(y^{\prime}, z,-\frac{p^{\prime}}{p_{n}}, \frac{1}{p_{n}}\right) \zeta_{i} \zeta_{j},
$$

where $p=\left(p_{1}, \ldots, p_{n}\right) \equiv\left(p^{\prime}, p_{n}\right), \zeta_{i}=\xi_{i}-p_{i} \xi_{n} / p_{n}$ for $i=1, \ldots, n-1$, and $\zeta_{n}=\xi_{n} / p_{n}$. Since (8.28) is a uniformly elliptic equation for $u=u_{0}:=\left(q_{0}^{-}-q_{0}^{+}\right) x_{n}$, it follows from (2.16) and (8.19) that (8.39) holds with the constants depending only on the data for any $v$ satisfying (8.34).

Now, using also the $C^{1, \alpha}$ dependence of $B(y, p)$ on $y$ which follows from $\varphi^{-} \in C^{2, \alpha}(\bar{\Lambda} \times[0, \infty))$ and $\Psi \in C^{2, \alpha}\left(\mathbf{R}^{n}\right)$, the standard estimates for elliptic equations imply the interior regularity $v \in C^{2, \alpha}(\Lambda \times(0, \infty))$ with

$$
\left|D^{2} v(y)\right| \leq C \sigma d_{y}^{\alpha-1} \quad \text { for } d_{y}=\operatorname{dist}\left(y, \partial\left(\Lambda \times \mathbf{R}_{+}\right)\right) .
$$

Note that the functions $B\left(y^{\prime}, z, P\right)$ and $\tilde{F}\left(y^{\prime}, z\right)$ and the domain $\Lambda \times(0, \infty)$ in (8.37) are independent of a solution $\varphi$ of Problem (TN).

Conversely, let $v(y)$ is a solution of (8.37) satisfying (8.34) and (8.35) with sufficiently small $\sigma$ depending only on the data so that (8.33) holds. Then the corresponding solution $\varphi$ of Problem (TN), satisfying (2.24), can be determined. Indeed, from (8.33),

$$
\mathcal{C}^{+}:=\left\{\left(x^{\prime}, v\left(x^{\prime}, y_{n}\right)\right): x^{\prime} \in \Lambda, y_{n}>0\right\}
$$


has the form (8.14), and a function $\tilde{u} \in C^{1, \alpha}\left(\overline{\mathcal{C}^{+}}\right)$can be defined such that (8.31) holds. Then (8.27) holds. Moreover, (8.34) implies (8.15). Next, we conclude that $\Omega^{+}:=\Psi\left(\mathcal{C}^{+}\right)$has the form (2.23) and (2.30), and $u(x):=\tilde{u}(\Phi(x))$ satisfies (8.12). Moreover, (8.15) implies (8.7). If $\sigma$ is sufficiently small, then (8.7) and (8.15) imply (8.8) for $u$ and $\tilde{u}$, respectively. Now, (8.35) implies (8.16) and further (8.9). Then it follows that $\varphi$, defined by $\varphi=\varphi^{-}-u$ in $\Omega^{+}$and $\varphi=\varphi^{-}$in $\Omega \backslash \Omega^{+}$, is a weak solution of Problem (TN) satisfying (2.24).

Thus, the uniqueness of solutions of the conormal problem (8.37) satisfying (8.34) and (8.35) implies the uniqueness of solutions of Problem (TN) satisfying (2.24).

8.3. Uniqueness of Weak Solutions of the Conormal Problem (8.37) Satisfying (8.34) and (8.35). Suppose $v_{1}$ and $v_{2}$ are two solutions of $(8.37)$ in $\Lambda \times(0, \infty)$ satisfying (8.34) and (8.35). We assume that $\sigma$ is small so that (8.33) holds for $v_{1}$ and $v_{2}$. Consider the function $w=v_{2}-v_{1}$. It satisfies

$$
\|w\|_{1, \alpha, \Lambda \times(0, \infty)} \leq 2 C \sigma
$$

and

$$
\|w\|_{1,0, \Lambda \times(R, \infty)} \rightarrow 0 \quad \text { as } R \rightarrow \infty .
$$

Also, subtracting equations (8.37) for $v_{1}$ and $v_{2}$, we get

$$
\int_{\Lambda \times(0, \infty)}\left(\sum_{i, j=1}^{n} a_{i j}(y) w_{y_{j}} \varphi_{y_{i}}+\sum_{i=1}^{n} b_{i}(y) w \varphi_{y_{i}}\right) d y=0
$$

for any $\varphi \in C_{c}^{1}\left(\mathbf{R}^{n}\right)$, where $a_{i j}$ and $b_{i}$ are defined by

$$
\begin{gathered}
a_{i j}(y)=\int_{0}^{1} B_{p_{j}}^{i}\left(y^{\prime},(1-t) v_{1}(y)+t v_{2}(y),(1-t) D v_{1}(y)+t D v_{2}(y)\right) d t, \\
b_{i}(y)=\int_{0}^{1}\left(B_{z}^{i}\left(y^{\prime},(1-t) v_{1}(y)+t v_{2}(y),(1-t) D v_{1}(y)+t D v_{2}(y)\right)\right. \\
\left.\quad+\delta_{i}^{n} \partial_{n} \tilde{F}\left(y^{\prime},(1-t) v_{1}(y)+t v_{2}(y)\right)\right) d t .
\end{gathered}
$$

Note that $a_{i j}, b_{i} \in C^{\alpha}(\bar{\Lambda} \times[0, \infty))$ with

$$
\left|a_{i j}(y)\right|+\left|b_{i}(y)\right| \leq C \quad \text { for any } y \in \Lambda \times(0, \infty), \quad i, j=1, \ldots, n,
$$

which follows from (8.2), (8.11), (8.19), (8.24), (8.33), (8.34), and (8.38). Moreover, using (8.3), (8.20), and (8.26), we see that

$$
b^{i} \equiv 0 \quad \text { on } \quad\left\{y_{n}>L_{1}\right\} \quad \text { for } i=1, \ldots, n,
$$

where $L_{1}=2 L /\left(q_{0}^{-}-q_{0}^{+}\right)$. Also, using (8.40) for $v_{1}$ and $v_{2}$ yields

$$
\left|D^{2} v(y)\right| \leq C \sigma d_{y}^{\alpha-1}, \quad \text { where } d_{y}=\operatorname{dist}(y, \partial(\Lambda \times(0, \infty)))
$$

Note that, by (8.39), the coefficients $a_{i j}(y)$ satisfy the ellipticity condition (3.35) with constant $\tilde{\lambda}$ for any $y \in \Lambda \times(0, \infty)$.

We first prove that $w \geq 0$ by choosing the following test function $\varphi$ in (8.44): For fixed $\varepsilon>0$ and $R>0$,

where

$$
\varphi=\frac{\left(w-m_{R}\right)^{+}}{\left(w-m_{R}\right)^{+}+\varepsilon},
$$

$$
m_{R}:=\max \left(0, \sup _{y \in \Lambda \times(R, \infty)} w(y)\right)<\infty
$$


by (8.42). Then $\varphi \in C^{0,1}(\bar{\Lambda} \times[0, \infty)$ and $\varphi \equiv 0$ on $\Lambda \times[R, \infty)$. Thus we can substitute this $\varphi$ into (8.44) to obtain

$$
\int_{\Lambda \times(0, R)}\left(\frac{\varepsilon a_{i j} w_{y_{i}}^{+}\left(\left(w-m_{R}\right)^{+}\right)_{y_{j}}}{\left(\left(w-m_{R}\right)^{+}+\varepsilon\right)^{2}}+\frac{\varepsilon b_{j} w^{+}\left(\left(w-m_{R}\right)^{+}\right)_{y_{j}}}{\left(\left(w-m_{R}\right)^{+}+\varepsilon\right)^{2}}\right) d y \leq 0 .
$$

Then using the ellipticity, (8.47), and (8.46) yields

$$
\begin{aligned}
& \lambda \int_{\Lambda \times(0, R)}\left|D \log \left(1+\left(w-m_{R}\right)^{+} / \varepsilon\right)\right|^{2} d y=\lambda \int_{\Lambda \times(0, R)} \frac{\left|D\left(w-m_{R}\right)^{+}\right|^{2}}{\left(\left(w-m_{R}\right)^{+}+\varepsilon\right)^{2}} d y \\
& \leq \int_{\Lambda \times(0, R)}\left(\frac{b_{j}\left(w-m_{R}\right)^{+}\left(\left(w-m_{R}\right)^{+}\right)_{y_{j}}}{\left(\left(w-m_{R}\right)^{+}+\varepsilon\right)^{2}}\right. \\
& \left.\quad+m_{R} b_{j}\left(\frac{\left(w-m_{R}\right)^{+}}{\left(w-m_{R}\right)^{+}+\varepsilon}\right)_{y_{j}}\right) d y \\
& \leq C \int_{\Lambda \times\left(0, L_{1}\right)}\left|D \log \left(1+\left(w-m_{R}\right)^{+} / \varepsilon\right)\right| d y \\
& \quad+m_{R} \int_{\partial\left[\Lambda \times\left(0, L_{1}\right)\right]}|b| d S+m_{R} \int_{\Lambda \times\left(0, L_{1}\right)}|D b| d y \\
& \leq C\left(\int_{\Lambda \times\left(0, L_{1}\right)}\left|D \log \left(1+\left(w-m_{R}\right)^{+} / \varepsilon\right)\right|^{2} d y\right)^{1 / 2}+C
\end{aligned}
$$

where we used (8.45) and (8.48) in the last estimate. Thus, for $R>L_{1}$,

$$
\int_{\Lambda \times(0, R)}\left|D \log \left(1+\left(w-m_{R}\right)^{+} / \varepsilon\right)\right|^{2} d y \leq C .
$$

Since $\log \left(1+\left(w-m_{R}\right)^{+} / \varepsilon\right)=0$ on $\Lambda \times\left\{y_{n}=R\right\}$, it follows from the Poincaré inequality that

$$
\int_{\Lambda \times(0, R)}\left|\log \left(1+\left(w-m_{R}\right)^{+} / \varepsilon\right)\right|^{2} d y \leq C
$$

where $C$ depends on the data and $R$, but is independent of $\varepsilon$. Letting $\varepsilon \rightarrow 0$, we conclude $\left(w-m_{R}\right)^{+} \equiv 0$ in $\Lambda \times(0, R)$, which, from the definition of $m_{R}$, implies $w \leq m_{R}$ in $\Lambda \times(0, \infty)$. Letting $R \rightarrow \infty$ and noting that $\lim _{R \rightarrow \infty} m_{R}=0$ by (8.43), we get $w \leq 0$ in $\Lambda \times(0, \infty)$. Similarly, $w \geq 0$ in $\Lambda \times(0, \infty)$. Thus the uniqueness is proved.

8.4. Stability. As a consequence of the uniqueness, nondegeneracy, and regularity of solutions of the free boundary problem, we have the following stability theorem.

Theorem 8.1. There exist a constant $\sigma_{0}>0$ and a nonnegative nondecreasing function $\mathcal{G} \in$ $C([0, \infty))$ with $\mathcal{G}(0)=0$, depending only on $n, \alpha, \gamma, C_{0}, \Lambda, L$, and $q_{0}^{+}$, such that, if

(i) $0<\kappa<\sigma<\sigma_{0}$;

(ii) $\Psi: \mathbf{R}^{n} \rightarrow \mathbf{R}^{n}$ satisfies (2.16), and (2.17), and $\hat{\Psi}: \mathbf{R}^{n} \rightarrow \mathbf{R}^{n}$ satisfies (2.17) and

$$
\|\Psi-\hat{\Psi}\|_{3, \alpha, \Lambda \times\left(-\frac{1}{2}, \frac{1}{2}\right)} \leq \kappa
$$

(iii) $\varphi^{-}(x)$ satisfies $(2.32)$ and $\hat{\varphi}^{-}(x)$ satisfies

$$
\left\|\varphi^{-} \circ \Psi^{-1}-\hat{\varphi}^{-} \circ \hat{\Psi}^{-1}\right\|_{2, \alpha, \Lambda \times\left(-\frac{1}{2}, \frac{1}{2}\right)} \leq \kappa,
$$

then the unique solutions $\varphi(x)$ and $\hat{\varphi}(x)$ of Problem $(\mathrm{TN})$ for the nozzles defined by the mappings $\Psi$ and $\hat{\Psi}$ in (2.14) and supersonic upstream flows $\varphi^{-}(x)$ and $\hat{\varphi}^{-}(x)$, respectively, satisfy

$$
\left\|\tilde{f}_{\varphi}-\tilde{f}_{\hat{\varphi}}\right\|_{1, \alpha, \Lambda} \leq \mathcal{G}(\kappa)
$$


where $\tilde{f}_{\varphi}\left(x^{\prime}\right)$ and $\tilde{f}_{\hat{\varphi}}\left(x^{\prime}\right)$ are the free boundary functions of the cylindric domains $\mathcal{C}^{+}(\varphi):=$ $\Phi\left(\Omega^{+}(\varphi)\right)$ and $\mathcal{C}^{+}(\hat{\varphi}(x)):=\hat{\Phi}\left(\Omega^{+}(\hat{\varphi})\right)$ in (8.14), respectively, where $\Phi=\Psi^{-1}$ and $\hat{\Phi}=\hat{\Psi}^{-1}$.

Its proof repeats the one of $[4$, Theorem 6.1]. See also the remarks on the proof of the stability part of [5, Theorem 2.3]. In the present case, as an additional step, the mapping from $\Omega$ to $\mathcal{C}$ is involved.

\section{Appendix A. Existence of Smooth Supersonic Upstream Flows in the Nozzle}

In this paper, we require a local $C^{1, \alpha}$ supersonic solution $\varphi^{-}$in Sections $3-7$ and $C^{2, \alpha}$ supersonic solution $\varphi^{-}$in Section 8 in the domain $\Omega_{1}:=\Omega \cap\left\{-1<x_{n} \leq 1\right\}$ of the initial-boundary value problem (2.18)-(2.19) for the nonlinear wave equation (1.1) such that

$$
\left\|\varphi^{-}-\varphi_{0}^{-}\right\|_{k, \alpha, \Omega_{1}} \leq C_{0} \sigma, \quad k=1 \text { or } 2
$$

for some $C_{0}>0$ independent of $\sigma$, when $\sigma$ is sufficiently small. This is a direct corollary of the standard local theory of smooth solutions of the initial-boundary value problem for the nonlinear wave equations.

Proposition A.1. Let the nozzle boundary satisfy (2.15) and (2.16). Let the Cauchy data $\left(\varphi_{e}^{-}, \psi_{e}^{-}\right)$on $\partial_{o} \Omega$ satisfies

$$
\left\|\varphi_{e}^{-}-q_{0}^{-} x_{n}\right\|_{H^{s+k}}+\left\|\psi_{e}^{-}-q_{0}^{-}\right\|_{H^{s+k-1}} \leq \sigma, \quad k=1 \text { or } 2,
$$

for some integer $s>n / 2+1$ and the compatibility conditions on the intersection $\overline{\partial_{l} \Omega} \cap \overline{\partial_{o} \Omega}$ up to the $(s+k)^{t h}$-order. Then there exist $\sigma_{0}>0$ and $C_{0}>0$ such that there exists a unique classical solution $\varphi^{-} \in C^{k+1}\left(\overline{\Omega_{1}}\right)$ of problem (2.18)-(2.19) for the nonlinear wave equation (1.1), respectively for $k=1$ or 2 , such that, when $\sigma \leq \sigma_{0}$,

$$
\left\|\varphi^{-}-\varphi_{0}^{-}\right\|_{k+1,0, \Omega_{1}} \leq C_{0} \sigma .
$$

If, in addition, the conditions (2.15), (2.16), and (A.2), as well as the compatibility conditions holds for higher order derivatives, then the solution $\varphi^{-}$has correspondingly higher regularity.

As in Section 8, we set

$$
w(x):=\varphi^{-}(x)-q_{0}^{-} x_{n} .
$$

Then $w$ satisfies the following initial-boundary value problem:

$$
\begin{aligned}
& \operatorname{div} A(D w)=0, \\
& \left.\left(w, w_{x_{n}}\right)\right|_{x_{n}=-1}=\left(\varphi_{e}^{-}+q_{0}^{-}, \psi_{e}^{-}-q_{0}^{-}\right), \\
& \left.w_{\nu}\right|_{\partial_{l} \Omega}=-q_{0}^{-} \nu_{n},
\end{aligned}
$$

as well as the compatibility conditions up to the $(s+k)^{t h}$-order, where

$$
A(P)=\rho\left(\left|P+q_{0}^{-} e_{n}\right|^{2}\right)\left(P+q_{0}^{-} e_{n}\right)-\rho\left(\left(q_{0}^{-}\right)^{2}\right) q_{0}^{-} e_{n} .
$$

Since $q_{0}^{-}>c_{*}$, then (A.4) is strictly hyperbolic, nonlinear wave equations with respect to the $x_{n}$-direction (whose role is as the time direction) for a small perturbation of the trivial solution $u=0$. By the standard energy estimates and the iteration procedure (cf. [28, 29, 31] and the references cited therein), then, for small $\sigma>0$, there exists a unique solution

$$
w\left(x^{\prime}, x_{n}\right) \in \cap_{j=0}^{s+k} C^{j}\left([-1,1] ; H^{s+1-j}(\bar{\Omega})\right)
$$

such that

$$
\sum_{j=0}^{s+k}\|w\|_{C^{j}\left([-1,1] ; H^{s+1-j}(\bar{\Omega})\right)} \leq C \sigma,
$$

for $k=1$ or 2 , respectively. Since $s>n / 2+1$, the Sobolev embedding theorem implies (A.3). 
Acknowledgments. Gui-Qiang Chen's research was supported in part by the National Science Foundation under Grants DMS-0244473, DMS-0204225, DMS-0204455, and INT-9987378 and an Alexandre von Humboldt Foundation Fellowship. Mikhail Feldman's research was supported in part by the National Science Foundation under Grants DMS-0200644 and DMS-0074037.

\section{REFERENCES}

[1] S. Agmon, A. Douglis, and L. Nirenberg, Estimates near the boundary for solutions of elliptic partial differential equations satisfying general boundary conditions I, Comm. Pure Appl. Math. 12 (1959), 623-727; II, Comm. Pure Appl. Math. 17 (1964), 35-92.

[2] L. Bers, Existence and uniqueness of subsonic flows past a given profile, Comm. Pure Appl. Math. 7 (1954), 441-504.

[3] S. Canić, B. L. Keyfitz, and G. Lieberman, A proof of existence of perturbed steady transonic shocks via a free boundary problem, Comm. Pure Appl. Math. 53 (2000), 484-511.

[4] G.-Q. Chen and M. Feldman, Multidimensional transonic shocks and free boundary problems for nonlinear equations of mixed type, J. Amer. Math. Soc. 16 (2003), 461-494.

[5] G.-Q. Chen and M. Feldman, Steady transonic shocks and free boundary problems in infinite cylinders for the Euler equations, Comm. Pure Appl. Math. 57 (2004), 310-356.

[6] G.-Q. Chen and M. Feldman, Free boundary problems and transonic shocks for the Euler equations in unbounded domains, Ann. Scuola Norm. Sup. Pisa Cl. Sci. (5), 4 (2004) (to appear).

[7] S.-X. Chen, Existence of stationary supersonic flows past a point body, Arch. Rational Mech. Anal. 156 (2001), 141-181.

[8] S.-X. Chen, Asymptotic behavior of supersonic flow past a convex combined wedge, Chinese Ann. Math. 19B (1998), 255-264.

[9] A. Constantin and W. Strauss, Exact steady periodic water waves with vorticity, Comm. Pure Appl. Math. 57 (2004), 481-527.

[10] M. Costabel and M. Dauge, Construction of corner singularities for Agmon-Douglis-Nirenberg elliptic systems, Math. Nachr. 162 (1993), 209-237.

[11] M. Costabel and M. Dauge, Stable asymptotics for elliptic systems on plane domains with corners, Commun. Partial Diff. Eqs. 19 (1994), 1677-1726.

[12] R. Courant and K. O. Friedrichs, Supersonic Flow and Shock Waves, Springer-Verlag: New York, 1948.

[13] C. M. Dafermos, Hyperbolic Conservation Laws in Continuum Physics, Springer-Verlag: Berlin, 2000.

[14] M. Dauge, Elliptic Boundary Value Problems in Corner Domains-Smoothness and Asymptotics of Solutions, Lecture Notes in Math. 1341, Springer-Verlag: Berlin, 1988.

[15] G. Dong, Nonlinear Partial Differential Equations of Second Order, Transl. Math. Monographs, 95, AMS: Providence, RI, 1991.

[16] P. Embid, J. Goodman, and A. Majda, Multiple steady state for 1-D transonic flow, SIAM J. Sci. Stat. Comp. 5 (1984), 21-41.

[17] R. Finn and D. Gilbarg, Asymptotic behavior and uniqueness of plane subsonic flows, Comm. Pure Appl. Math. 10 (1957), 23-63.

[18] R. Finn and D. Gilbarg, Three-dimensional subsonic flows, and asymptotic estimates for elliptic partial differential equations, Acta Math. 98 (1957), 265-296.

[19] C. A. J. Fletcher, Computational Techniques for Fluid Dynamics, Vols. I-II, Springer-Verlag: Berlin, 1991.

[20] D. Gilbarg and L. Hörmander, Intermediate Schauder estimates, Arch. Rational Mech. Anal. 74 (1980), 297-318.

[21] D. Gilbarg and N. Trudinger, Elliptic Partial Differential Equations of Second Order, 2nd Ed., SpringerVerlag: Berlin, 1983.

[22] J. Glimm and A. Majda, Multidimensional Hyperbolic Problems and Computations, Springer-Verlag: New York, 1991.

[23] J. Glimm, G. Marshall, and B. Plohr, A generalized Riemann problem for quasi-one-dimensional gas flow, Adv. Appl. Math. 5 (1984), 1-30.

[24] P. Grisvard, Elliptic Problems in Nonsmooth Domains, Monographs and Studies in Math. 24, Pitman: London, 1985.

[25] C.-H. Gu, A method for solving the supersonic flow past a curved wedge (in Chinese), Fudan J. 7 (1962), 11-14.

[26] Q. Han and F. Lin, Elliptic Partial Differential Equations, Courant Lecture Notes in Mathematics 1. Courant Institute of Mathematical Sciences: New York; American Mathematical Society (AMS): Providence, 1997.

[27] H. Glaz and T.-P. Liu, The asymptotic analysis of wave interactions and numerical calculations of transonic nozzle flow, Adv. Appl. Math. 5 (1984), 114-146.

[28] F. John, Nonlinear Wave Equations, Formation of Singularities, University Lecture Series 2, American Mathematical Society (AMS): Providence, 1990. 
[29] M. Kikuchi and Y. Shibata, On the mixed problem for some quasilinear hyperbolic system with fully nonlinear boundary condition, J. Diff. Eqs. 80 (1989), 154-197.

[30] D. Kinderlehrer and L. Nirenberg, Regularity in free boundary problems, Ann. Scuola Norm. Sup. Pisa Cl. Sci. (4), 4 (1977), 373-391.

[31] H. Koch, Mixed problems for fully nonlinear hyperbolic equations, Math. Z. 214 (1993), 9-42.

[32] P. D. Lax, Hyperbolic Systems of Conservation Laws and the Mathematical Theory of Shock Waves, CBMSRCSM, SIAM: Philiadelphia, 1973.

[33] T.-T. Li, On a free boundary problem, Chinese Ann. Math. 1 (1980), 351-358.

[34] T.-T. Li and W.-C. Yu, Boundary Value Problems for Quasilinear Hyperbolic Systems, Duke University Mathematics Series, V. Durham, NC 27706: Duke University, Mathematics Department. X, 1985.

[35] G. Lieberman, Mixed boundary value problems for elliptic and parabolic differential equations of second order, J. Math. Anal. Appl. 113 (1986), 422-440.

[36] G. Lieberman, Hölder continuity of the gradient of solutions of uniformly parabolic equations with conormal boundary conditions, Ann. Mat. Pura Appl. (4), 148 (1987), 77-99.

[37] G. Lieberman, Oblique derivative problems in Lipschitz domains II: Discontinuous boundary data. J. Reine Angew. Math. 389 (1988), 1-21.

[38] G. Lieberman, Second Order Parabolic Differential Equations, World Scientific Publishing Co. Inc.: River Edge, NJ, 1996.

[39] G. Lieberman and N. Trudinger, Nonlinear oblique boundary value problems for nonlinear elliptic equations, Trans. Amer. Math. Soc. 295 (1986), 509-546.

[40] W.-C. Lien and T.-P. Liu, Nonlinear stability of a self-similar 3-dimensional gas flow, Commun. Math. Phys. 204 (1999), 525-549.

[41] T.-P. Liu, Nonlinear stability and instability of transonic gas flow through a nozzle, Comm. Math. Phys. 83 (1983), 243-260.

[42] T.-P. Liu, Nonlinear resonance for quasilinear hyperbolic equation, J. Math. Phys. 28 (1987), 2593-2602.

[43] C. S. Morawetz, On the non-existence of continuous transonic flows past profiles I-III, Comm. Pure Appl. Math. 9 (1956), 45-68; 10 (1957), 107-131; 11 (1958), 129-144.

[44] C. S. Morawetz, On a weak solution for a transonic flow problem, Comm. Pure Appl. Math. 38 (1985), $797-817$.

[45] D. G. Schaeffer, Supersonic flow past a nearly straight wedge, Duke Math. J. 43 (1976), 637-670.

[46] M. Shiffman, On the existence of subsonic flows of a compressible fluid, J. Rational Mech. Anal. 1 (1952), 605-652.

[47] E. M. Stein, Singular Integrals and Differentiability Properties of Functions, Princeton Univ. Press: Princeton, NJ, 1970.

[48] G. B. Whitham, Linear and Nonlinear Waves, John Wiley \& Sons: New York, 1974.

[49] Y. Zhang, Global existence of steady supersonic potential flow past a curved wedge with a piecewise smooth boundary, SIAM J. Math. Anal. 31 (1999), 166-183.

[50] Y. Zheng, Systems of Conservation Laws: Two-dimensional Riemann Problems, Birkhäuser Boston, Inc.: Boston, MA, 2001.

Department of Mathematics, Northwestern University, Evanston, IL 60208-2730

E-mail address: gqchen@math.northwestern.edu

Department of Mathematics, University of Wisconsin, Madison, Wi 53706

E-mail address: feldman@math.wisc.edu 